\title{
Base change and Grothendieck duality for Cohen-Macaulay maps
}

\author{
Pramathanath Sastry
}

\begin{abstract}
Let $f: X \rightarrow Y$ be a Cohen-Macaulay map of finite type between Noetherian schemes, and $g: Y^{\prime} \rightarrow Y$ a map, with $Y^{\prime}$ Noetherian. Let $f^{\prime}: X^{\prime} \rightarrow Y^{\prime}$ be the base change of $f$ under $g$ and $g^{\prime}: X^{\prime} \rightarrow X$ the base change of $g$ under $f$. We show that there is a canonical isomorphism $\theta_{g}^{f}: g^{\prime *} \omega_{f} \simeq \omega_{f^{\prime}}$, where $\omega_{f}$ and $\omega_{f^{\prime}}$ are the relative dualizing sheaves. The map $\theta_{g}^{f}$ is easily described when $f$ is proper, and has a more subtle description when $f$ is not. If $f$ is smooth we show that $\theta_{g}^{f}$ corresponds to the canonical identification $g^{\prime *} \Omega_{f}^{r}=\Omega_{f^{\prime}}^{r}$ of differential forms, where $r$ is the relative dimension of $f$. This work is closely related to B. Conrad's work on base change. However, our approach to the problems and our viewpoint are very different from Conrad's: dualizing complexes and their Cousin versions, residual complexes, do not appear in this paper.
\end{abstract}

\section{Introduction}

Our approach to Grothendieck duality is the approach of Deligne and Verdier [Del66, Ver69] with important elaborations by Alonso et al.. [AJL99]. In particular, we do not use residual complexes or dualizing complexes, which are crucial ingredients in the approach laid out in Hartshorne's voluminous book [Har66]. We will be examining the behavior of relative duality for a CohenMacaulay map $f: X \rightarrow Y$ of Noetherian schemes. Our intent is to show that the main result of Conrad's recent book [Con00] on Grothendieck duality concerning base change for relative dualizing sheaves and traces for Cohen-Macaulay maps (see [Con00, pp. 172-173, Theorem 3.6.5]) can be established without recourse to dualizing or residual complexes.

Our approach is the 'soft' approach, with emphasis being placed on functorial aspects (i.e. on how 'upper shriek' behaves and what it does, rather than its precise description via residual complexes). In the case where the map $f: X \rightarrow Y$ is smooth, our techniques also establish the commutativity of diagram (1.1.3) of [Con00], one of the main motivations for Conrad's book.

A word about our philosophy. We have already pointed out our functorial bent. In other words we would like to believe that there is so much information built into the abstract definitions of the various objects associated with duality theory, that explicit information can be elicited with minimum computations. For a practitioner of such a philosophy the hard work is in finding the intricate web of relationships between the abstractly defined objects (e.g. the pseudofunctoriality of $(\cdot)^{!}$, and compatibility with (Zariski, étale, smooth, flat) localizations). Lipman's notes [Lip03] give an indication of the effort involved in getting the setup right. While the effort involved can be (and is) considerable, it often results in conceptual clarifications. At the concrete level, computations do occur in duality theory. In such instances we give primacy to residues rather than residual complexes and in general, we believe, this can always be done. In sharp contrast, Conrad uses

Received 7 December 2001, accepted in final form 2 October 2002.

2000 Mathematics Subject Classification 14F05 (primary), 14A15, 14F10, 18E30 (secondary).

Keywords: residues, Grothendieck duality, Kleiman's $r$-dualizing pairs, Cohen-Macaulay maps.

This journal is (C) Foundation Compositio Mathematica 2004. 


\section{P. SASTRY}

residual complexes. If one shows that residues (rather than traces) behave well with respect to base change of Cohen-Macaulay maps, then local duality gives the rest. We leave it to the reader to judge if our approach results in conceptual clarifications.

From our point of view, the general base change theorem for Cohen-Macaulay maps is a careful functorial upgrading of the following easy base change formula

$$
\operatorname{Hom}_{A}(B, A) \otimes_{A} A^{\prime}=\operatorname{Hom}_{A^{\prime}}\left(B^{\prime}, A^{\prime}\right),
$$

where $A \rightarrow B$ is a finite, flat map of Noetherian rings, $A^{\prime}$ is an $A$-algebra and $B^{\prime}=B \otimes_{A} A^{\prime}$. This upgrading requires establishing local duality and setting up the right functorial machinery. Note that formula (1.1) says that the relative dualizing sheaf for a finite flat map of Noetherian schemes $f: X \rightarrow Y$ (i.e. a proper Cohen-Macaulay map of relative dimension 0) behaves well with respect to base change and so does trace ('evaluation at 1'). Conrad informs me that the above formula is also the key to his approach.

We have included a number of appendices giving (hopefully) something of a coherent survey of facts scattered in the literature regarding base change for direct images with support and for local cohomology. We have only included those facts which are needed for this paper. Appendices are labeled by the upper case letters in the English alphabet, and so one has to look at Appendix A for (say) Proposition A.1.3.

In a short while we will give a quick summary of the Deligne-Verdier (DV) approach to duality. The classic references are [Del66] and [Ver69]. Deligne's and Verdier's results apply to (finite-type, separated) maps between Noetherian schemes of finite Krull dimension. This is generalized to arbitrary Noetherian schemes by Alonso, Jeremías and Lipman in [AJL99]. In fact, their results are far more general than we need in this paper. They work with Noetherian formal schemes. Since our interest is not restricted to schemes with finite Krull dimension, we will appeal to [AJL99] for our results (and make a respectful bow towards [Del66] and [Ver69] by also giving appropriate references to the analogous results there). The key results in the DV approach to duality are: (a) the existence of a right adjoint to the (derived) direct image functor for a proper map - the twisted inverse image functor in Verdier's terminology [Del66, pp. 416-417], [Ver69, pp. 393-394, Theorem 1], [AJL99, p. 5, Theorem 1] and [Lip99, p. 120, Corollary (4.3)]; (b) compatibility of this twisted inverse image functor with flat base change [Ver69, pp. 394-395, Theorem 2], [AJL99, pp. 8-9, Theorem 3]; and as a consequence (c) the localness of the twisted inverse image functor [Ver69, p. 395, Corollary 1], [AJL99, p. 88, Proposition 8.3.1]. We should point out that Neeman has an intriguingly different approach to the above results (see [Ne96]).

\subsection{Notation and terminology}

Schemes will mean Noetherian schemes. For any scheme $Z, Z_{\mathrm{qc}}$ (respectively $Z_{c}$ ) will denote the category of quasi-coherent $\mathcal{O}_{Z}$-modules (respectively coherent $\mathcal{O}_{Z}$-modules). The category whose objects are complexes of $\mathcal{O}_{Z}$-modules whose cohomologies are quasi-coherent and vanish in sufficiently negative degrees and whose morphisms are homotopy equivalence classes of maps of complexes will be denoted $K_{\mathrm{qc}}^{+}(Z)$. The corresponding derived category (obtained by formally inverting quasi-isomorphisms) will be denoted $D_{\mathrm{qc}}^{+}(Z)$. The resulting localization functor will be denoted

$$
Q_{Z}: K_{\mathrm{qc}}^{+}(Z) \rightarrow D_{\mathrm{qc}}^{+}(Z)
$$

Recall that an injective object of $Z_{\mathrm{qc}}$ is an injective object in the category of $\mathcal{O}_{Z}$-modules (since $Z$ is Noetherian), and hence is flasque. By an injective sheaf on $Z$ we will always mean an injective object in $Z_{\mathrm{qc}}$. Higher direct images (with or without supports) of maps of schemes $h: Z \rightarrow W$ will, unless otherwise stated, be thought of as functors on $Z_{\mathrm{qc}}$. In view of the previous comment, and the existence of enough injectives in $Z_{\mathrm{qc}}$, it is simply the restriction of the usual higher direct 


\section{Base Change For COHEN-MaCAulay MaPs}

images to $Z_{\mathrm{qc}}$. In particular, a statement of the form $R^{i} h_{*}=0$ means that it is zero when applied to objects in $Z_{\mathrm{qc}}$ (though perhaps not zero when applied to an arbitrary sheaf of abelian groups).

\subsection{Sign conventions for complexes}

We follow the following (standard) sign conventions. These differ somewhat from the (non-standard) conventions in [Har66]. If $A^{\bullet}$ and $B^{\bullet}$ are complexes in an abelian category $\mathcal{A}$ which admits infinite direct sums and a tensor product $\otimes$, then the following hold.

i) $\operatorname{Hom}^{\bullet}\left(A^{\bullet}, B^{\bullet}\right)$ is the complex whose $n$th graded piece is

$$
\operatorname{Hom}^{n}\left(A^{\bullet}, B^{\bullet}\right)=\operatorname{Hom}_{\mathrm{gr}}\left(A^{\bullet}, B^{\bullet}[n]\right),
$$

where Hom $_{\text {gr }}$ denotes graded maps of degree zero. The differential follows the rule

$$
\begin{aligned}
d^{n} f & =d_{B} \circ-(-1)^{n} f \circ d_{A} \\
& =(-1)^{n+1}\left(f \circ d_{A}-d_{B[n]^{\circ}} f\right) .
\end{aligned}
$$

ii) $A^{\bullet} \otimes B^{\bullet}$ is the complex whose $n$th piece is

$$
\left(A^{\bullet} \otimes B^{\bullet}\right)^{n}=\oplus_{p \in \mathbb{Z}} A^{p} \otimes B^{n-p}
$$

and the differential is

$$
d^{n} \mid A^{p} \otimes B^{n-p}=d_{A}^{p} \otimes 1+(-1)^{p} \otimes d_{B}^{n-p} .
$$

iii) We have an isomorphism

$$
\theta_{i j}: A^{\bullet}[i] \otimes B^{\bullet}[j] \stackrel{\sim}{\longrightarrow}\left(A^{\bullet} \otimes B^{\bullet}\right)[i+j],
$$

which is 'multiplication by $(-1)^{p j}$, on $A^{p+i} \otimes B^{q+j}$. Note that $\theta_{i j}=\theta_{i 0}[j] \circ \theta_{0 j}$, but $\theta_{i j}=$ $(-1)^{i j} \theta_{0 j}[i]_{\circ} \theta_{i 0}$. See $\left[\operatorname{Lip} 03\right.$, p. 20, (1.5.4)] for the reason for introducing $\theta_{i j}$.

iv) We use the standard identification, the one without the intervention of signs (cf. [Con00, p. 7, (1.3.1)]), of $H^{i}\left(A^{\bullet}[r]\right)$ with $H^{i+r}\left(A^{\bullet}\right)$. This identification is functorial in $A^{\bullet}$. In other words we have an identity of functors

$$
H^{i}((\cdot)[r])=H^{i+r}(\cdot) .
$$

The identity (1.2) is robust under translations. In other words, the two identifications of $H^{i}\left(A^{\bullet}[r][s]\right)$ with $H^{i+r+s}\left(A^{\bullet}\right)$ (i.e. by either using (1.2) twice or by using $A^{\bullet}[r][s]=A^{\bullet}[r+s]$ and then applying (1.2)) agree.

Now suppose $R$ is a (commutative) ring. If $P$ is a finitely generated projective module, we identify $P$ with its double dual in the standard way. Let $P^{\bullet}$ be a complex of finitely generated projective modules over $R$. Then we check (using the conventions above) the following.

1) the complex $\widetilde{P}^{\bullet}=\operatorname{Hom}_{R}^{\bullet}\left(\operatorname{Hom}_{R}^{\bullet}\left(P^{\bullet}, R\right), R\right)$ has as its differentials the negatives of the differentials of $P^{\bullet}$. There is an isomorphism of complexes $P^{\bullet} \stackrel{\sim}{\longrightarrow} \widetilde{P}^{\bullet}$ given by multiplication by $(-1)^{n}$ at degree $n$.

2) If $Q^{\bullet}$ is the complex obtained from $\operatorname{Hom}_{R}^{\bullet}\left(P^{\bullet}, R\right)$ by changing all the differentials to their negatives, then

$$
P^{\bullet}=\operatorname{Hom}_{R}^{\bullet}\left(Q^{\bullet}, R\right) .
$$

3) If $M^{\bullet}$ is a complex of $R$-modules, the natural isomorphism of $R$-modules

$$
M^{p} \otimes_{R} \operatorname{Hom}_{R}\left(P^{s}, R\right) \stackrel{\sim}{\longrightarrow} \operatorname{Hom}_{R}\left(P^{s}, M^{p}\right)
$$

gives (without auxiliary signs) an isomorphism of complexes

$$
M^{\bullet} \otimes_{R} \operatorname{Hom}_{R}^{\bullet}\left(P^{\bullet}, R\right) \stackrel{\sim}{\longrightarrow} \operatorname{Hom}_{R}^{\bullet}\left(P^{\bullet}, M^{\bullet}\right),
$$

provided $M^{\bullet}$ or $P^{\bullet}$ is bounded. Note the order in which the tensor product is taken. 


\section{P. SASTRY}

We end this subsection with a discussion concerning translates and derived functors. A clarification becomes necessary in view of the convention adopted by Conrad in [Con00, p. 151, (3.4.13)]. One of our aims is to establish (1.4), which on first glance seems obvious, but which needs explication. If $\mathcal{A}$ is an abelian category with enough injectives, $F: \mathcal{A} \rightarrow \mathcal{B}$ a covariant left exact functor $(\mathcal{B}$ another abelian category) and $A$ is an object in $\mathcal{A}$, then (1.2) allows us to make an identification

$$
H^{i}(R F(A[r]))=R^{i+r} F(A)
$$

in the following way: choose an injective resolution $I^{\bullet}$ of $A$ (in degrees $\geqslant 0$ ), and use the induced injective resolution $I^{\bullet}[m]$ of $A[m]$ to compute derived functors of $A[m]$ for any $m$. Then, as in [Con00, p. 8, (1.3.4)], we have

$$
H^{i}(R F(A[r]))=H^{i}\left(F I^{\bullet}[r]\right)=H^{i+r}\left(F I^{\bullet}\right)=R^{i+r} F(A) .
$$

Now suppose we have a map in the derived category of complexes in $\mathcal{B}$,

$$
f: R F(A[r]) \rightarrow B[0]
$$

for some object $B$ in $\mathcal{B}$. Let $s$ be any integer. We claim that under the identification (1.3) we have the following equality of maps (with source $R^{r} F(A)$ and target $B$ ):

$$
H^{-s}(f[s])=H^{0}(f) .
$$

The claim is proved as follows. There exists a complex $C^{\bullet}$ in $\mathcal{B}$ which, though not necessary for what follows, may be taken to be bounded below, and maps of complexes $g: C^{\bullet} \rightarrow B[0]$ and $u: C^{\bullet} \rightarrow F\left(I^{\bullet}\right)[r]$, with $u$ a quasi-isomorphism, such that in the derived category of complexes in $\mathcal{B}$ we have (with localization functors suppressed) $f=g \circ u^{-1}$. It follows that $f[s]=g[s] \circ u[s]^{-1}$. By (1.2) we have

$$
H^{-s}(g[s]) \circ H^{-s}(u[s])^{-1}=H^{0}(g) \circ H^{0}(u)^{-1},
$$

giving (1.4). Note that the robustness of (1.2) under translation reconciles any ambiguity in the interpretation of (1.4) (see the comment immediately following (1.2)).

In the special case of $F=f_{*}$ where $f: X \rightarrow Y$ is a smooth proper map of relative dimension $r$, and $A=\wedge_{\mathcal{O}_{X}}^{r} \Omega_{X / Y}^{1}$ (the relative canonical sheaf), (1.3) differs from the convention adopted by Conrad in [Con00, p. 151, (3.4.13)] by a sign (if $i=0$, the sign is $(-1)^{r}$ ).

\subsection{Summary of the DV approach}

Here is the promised summary of the key points of the DV approach. Let $f: X \rightarrow Y$ be a separated finite type map of schemes. One 'constructs' a functor $f^{!}: D_{\mathrm{qc}}^{+}(Y) \rightarrow D_{\mathrm{qc}}^{+}(X)$ in two steps. If $f$ is proper, then $f^{!}$is defined as the right adjoint to $R f_{*}: D_{\mathrm{qc}}^{+}(X) \rightarrow D_{\mathrm{qc}}^{+}(Y)$ (which is shown to exist). If $f$ is not proper, then we pick a compactification $\bar{f}: \bar{X} \rightarrow Y$ of $f$, and $f^{!}$is defined to be $i^{*} \circ \bar{f}^{!}$, where $i: X \hookrightarrow \bar{X}$ is the natural inclusion. The local nature of $f^{!}$(see Remark 1.3.1 below) ensures that the end result is canonically independent of the compactification $\bar{f}$. Recall that Nagata's result in [Nag62] ensures the existence of a compactification of $f$. Nagata's proof is difficult to follow since it does not use the language of schemes. There are proofs of Nagata's result in the language of schemes, such as those by Lütkebohmert [Lut93] and an old unpublished one by Deligne written up by Conrad [Con].

The localness of 'upper shriek' needs, in a critical way, the results in [Ver69] and [AJL99] concerning flat base change and the twisted inverse image functor, therefore we quickly review this. First note that if $f: X \rightarrow Y$ is proper, then we have a natural transformation

$$
T_{f}: R f_{*} f^{!} \rightarrow 1_{D_{\mathrm{qc}}^{+}(Y)}
$$




\section{Base Change For COHEN-MaCAulay MaPS}

(the trace map) corresponding to the identity morphism $f^{!} \rightarrow f^{!}$. Let

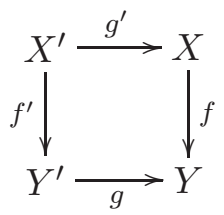

be a cartesian diagram with $g$ flat and $f$ proper. We have a composite map

$$
g^{\#} T_{f}: R f_{*}^{\prime} g^{\prime *} f^{!} \stackrel{\sim}{\longrightarrow} g^{*} R f_{*} f^{!} \stackrel{g^{*} T_{f}}{\longrightarrow} g^{*} .
$$

(See Remark B.2.2 in Appendix B for the isomorphism.) By the universal property of $\left(f^{\prime}, T_{f^{\prime}}\right)$ we get a unique map

$$
\Theta_{g}^{f}: g^{\prime *} f^{!} \longrightarrow f^{\prime !} g^{*}
$$

such that $T_{f^{\prime} \circ R f_{*}} \Theta_{g}^{f}=g^{\#} T_{f}$. The flat base change assertion in [Ver69, pp. 393-395, Theorem 2] and [AJL99, pp. 8-9, Theorem 3] is that $\Theta_{g}^{f}$ is an isomorphism.

Remark 1.3.1. Here is how the local nature of 'upper shriek' is proved using flat base change of proper maps. Suppose first that we have two compactifications $\left(\imath_{k}, f_{k}: X_{k} \rightarrow Y\right)$ of $f$ and that these compactifications can be embedded in a commutative diagram

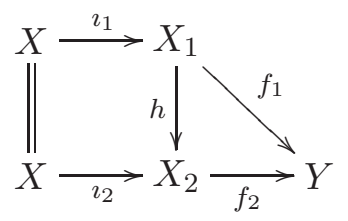

such that the square is cartesian. The last condition is satisfied, for example, when $\imath_{1}: X \hookrightarrow X_{1}$ is scheme theoretically dense. Indeed, if this is so, the natural map $X \rightarrow h^{-1}(X)$ is scheme theoretically dense, and being proper (for the composite $X \rightarrow h^{-1}(X) \stackrel{h}{\longrightarrow} X$ is the identity map, which is proper) is an isomorphism, proving that the square in (1.7) is cartesian in this case. Next, since $f_{1}^{!} \simeq h^{!} f_{2}^{!}$ (we are using the isomorphism $R f_{1 *} \simeq R f_{2 *} h_{*}$ and the adjointness of 'upper shriek' and 'derived lower star'), by flat base change we have

$$
\imath_{1}^{*} f_{1}^{!} \stackrel{\sim}{\longrightarrow} \imath_{1}^{*} h^{!} f_{2}^{!} \stackrel{\sim}{\longrightarrow} 1_{X}^{!} \imath_{2}^{*} f_{2}^{!}=\imath_{2}^{*} f_{2}^{!} .
$$

One checks from the definitions that this isomorphism has another description which is more useful at times. Let $T_{h}^{\prime}: R h_{*} f_{1}^{!} \rightarrow f_{2}^{!}$be the map that arises from the isomorphism $f_{1}^{!} \simeq h^{!} f_{2}^{!}$and $T_{h}$. Then the above isomorphism can be described by

$$
\imath_{1}^{*} f_{1}^{!}=\imath_{2}^{*} R h_{*} f_{1}^{!} \stackrel{\imath_{2}^{*} T_{h}^{\prime}}{\longrightarrow} \imath_{2}^{*} f_{2}^{!} .
$$

This latter description is used in the proof of Proposition 3.1.1 and in Proposition 4.2.1.

We have assumed that $f_{1}$ and $f_{2}$ are related by a commutative diagram of the type (1.7) with $X=h^{-1}(X)$. The general case can be reduced to this by finding a third compactification $\left(\imath_{3}: X \hookrightarrow\right.$ $X_{3}, f_{3}: X_{3} \rightarrow Y$ ) of $f$ such that for $k=1,2$ we have a cartesian square of $Y$-schemes

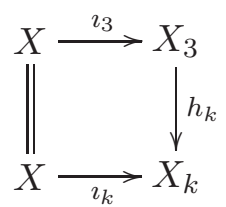

and then using the isomorphisms $\imath_{3}^{*} f_{3}^{!} \simeq \imath_{k}^{*} f_{k}^{!}$to get $\imath_{2}^{*} f_{2}^{!} \simeq \imath_{1}^{*} f_{1}^{!}$. Such a compactification can be found by setting $X_{3}$ equal to the scheme theoretic closure of the diagonal embedding $X \rightarrow X_{1} \times_{Y} X_{2}$ 


\section{P. SASTRY}

(and setting $\imath_{3}, h_{1}, h_{2}$ and $f_{3}$ equal to the obvious maps). Note that, with this choice of $X_{3}, X$ is scheme theoretically dense in $X_{3}$ and hence (1.9) is cartesian for $k=1,2$. It can be checked that the resulting isomorphism $\imath_{2}^{*} f_{2}^{!} \stackrel{\sim}{\longrightarrow} \imath_{1}^{*} f_{1}^{!}$is independent of the auxiliary data $\left(\imath_{3}, h_{1}, h_{2}, f_{3}\right)$.

If $\mu_{i j}: \imath_{j}^{*} f_{j}^{!} \stackrel{\sim}{\longrightarrow} \imath_{i}^{*} f_{i}^{!}$is the isomorphism described above for two compactifications $\left(\imath_{i}, f_{i}\right)$ and $\left(\imath_{j}, f_{j}\right)$ of $f$, then it is easy to see that:

a) $\mu_{i j}$ is compatible with open immersions into $X$;

b) for three compactifications, we have $\mu_{i j} \circ \mu_{j k}=\mu_{i k}$.

We should point out that in a different context (but with the same formalism) this has been worked out by Lipman in [Lip84, p. 46, Lemma (4.6)].

We can, in light of the above discussion, talk about $f$ ! even if $f$ is not proper (though separatedness is necessary). Moreover if $g: Y \rightarrow Z$ is another separated finite type map, then we have an isomorphism

$$
(g f)^{!} \stackrel{\sim}{\longrightarrow} f^{!} g^{!}
$$

arising, when $f$ and $g$ are proper, as the isomorphism adjoint to the isomorphism $R g_{*} \circ R f_{*} \stackrel{\sim}{\longrightarrow}$ $R(g f)_{*}$, and defined in general by compactifying and then restricting. One sees that (1.10) is independent of compactifications of $f$ and $g$ by considering diagrams as in [Del66, p. 413] (the order of $f$ and $g$ is reversed there), especially the second diagram (from which a south-west pointing arrow from $\bar{X}$ to $Y$ is missing). We leave the details to the reader. In conceptual terms we are talking about the pseudofunctoriality of $(\cdot)^{!}$which, roughly speaking, refers to the associativity of the isomorphism (1.10).

Remark 1.3.2. The $\mu_{i j}$ above are compatible with flat base change. More precisely, let $g: Y^{\prime} \rightarrow Y$ be a flat map and $(\cdot)^{\prime}=(\cdot) \times_{Y} Y^{\prime}$. Then the compatibility statement being made is that the diagram (with $(i, j)=(1,2)$ and $L g^{*}=g^{*}, L g^{\prime *}=g^{\prime *}$ etc.)

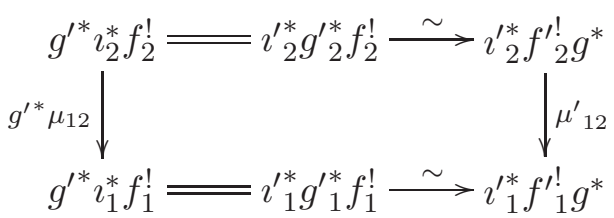

commutes, where the horizontal isomorphisms are $\imath_{k}^{*} \Theta_{g}^{f_{k}}, k=1,2$. By (1.6) and the 'associativity' of (1.10) we only need to check that diagram (1.11) commutes when $f_{1}$ dominates $f_{2}$, i.e. there is a map $h: X_{1} \rightarrow X_{2}$ such that (a) $f_{2} \circ h=f_{1}$ and $h^{-1}\left(\imath_{2}(X)\right)=\imath_{1}(X)$ (see Remark 1.3.1). In this situation, one checks that (1.11) commutes by verifying $\Theta_{\imath_{2}^{\prime}}^{h^{\prime}} \imath_{1}^{\prime *} \Theta_{g_{2}^{\prime}}^{h}=g^{\prime *} \Theta_{\imath_{2}}^{h}$ and, after making the identifications $f_{1}^{!}=h^{!} f_{2}^{!}$and $f^{\prime !}=h^{\prime !} f_{2}^{!}$, that $h^{\prime !} \Theta_{g}^{f_{2}} \circ \Theta_{g_{2}}^{h}=\Theta_{g}^{f_{1}}$ (i.e. (1.6) is compatible with (1.10)). These identities are proved in the appendices, the first in Remark B.2.4 and the second by the commutativity of the diagram (B.3). In view of the above discussion it makes sense to talk about $\Theta_{g}^{f}: g^{\prime *} f^{!} \rightarrow f^{\prime !} g^{*}$ for a separated finite type map $f: X \rightarrow Y$ and a flat map $g$ even when $f$ is not proper.

\subsection{The problem}

To explain the problem we will first consider a simpler situation, in which we have more hypotheses than we really need. Following [Con00], first consider a proper map $f: X \rightarrow Y$ which is CohenMacaulay of relative dimension $r$. The condition in italics means that:

a) $f$ is flat (of finite type) and

b) the non-empty fibers of $f$ are Cohen-Macaulay of pure dimension $r$. 


\section{Base Change for Cohen-Macaulay maps}

It is well known that in this situation, for $i \neq-r, H^{i}\left(f^{!} \mathcal{O}_{Y}\right)=0$ and hence $f^{!} \mathcal{O}_{Y} \stackrel{\sim}{\longrightarrow} \omega_{f}[r]$, where $\omega_{f}=H^{-r}\left(f^{!} \mathcal{O}_{Y}\right)\left[\operatorname{Lip} 79\right.$, p. 39, Lemma 1(i)]. Note that $\omega_{f}$ is coherent on $X$. It is further proved in [Lip79] that $\omega_{f}$ is flat over $Y$. It should be pointed out that the statement in [Lip79] is for $r=2$, but the proof works for arbitrary $r$.

Let

$$
\int_{f}=\int_{f}^{\mathcal{O}_{Y}}: R^{r} f_{*} \omega_{f} \rightarrow \mathcal{O}_{Y}
$$

be induced by the trace map $T_{f}: R f_{*} f^{!} \mathcal{O}_{Y} \rightarrow \mathcal{O}_{Y}$ (we remind the reader of our convention (1.3)). The pair $\left(\omega_{f}, \int_{f}\right)$ induces a functorial isomorphism $\operatorname{Hom}_{\mathcal{O}_{X}}\left(\mathcal{F}, \omega_{f}\right) \stackrel{\sim}{\longrightarrow} \operatorname{Hom}_{\mathcal{O}_{Y}}\left(R^{r} f_{*} \omega_{f}, \mathcal{O}_{Y}\right)$ for $\mathcal{F} \in X_{\mathrm{qc}}$, and hence $\left(\omega_{f}, \int_{f}\right)$ is unique up to unique isomorphism. Next suppose that $f$ is embedded in the cartesian square as follows.

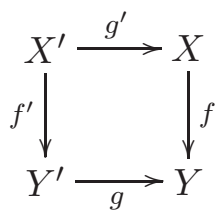

Since $R^{r} f_{*}(\cdot)$ commutes with arbitrary base change (see Proposition B.1.2), we see that the natural $\operatorname{map} g^{*} R^{r} f_{*} \omega_{f} \rightarrow R^{r} f_{*}^{\prime} g^{\prime *} \omega_{f}$ is an isomorphism. We therefore have a map

$$
g^{\#} \int_{f}: R^{r} f_{*}^{\prime} g^{\prime *} \omega_{f} \rightarrow \mathcal{O}_{Y^{\prime}}
$$

defined by the composite

$$
R^{r} f_{*}^{\prime} g^{\prime *} \omega_{f} \stackrel{\sim}{\longrightarrow} g^{*} R^{r} f_{*} \omega_{f} \stackrel{g^{*} \int_{f}}{\longrightarrow} g^{*} \mathcal{O}_{Y}=\mathcal{O}_{Y^{\prime}}
$$

The universal property of $\left(\omega_{f^{\prime}}, \int_{f^{\prime}}\right)$ (note that $f^{\prime}$ is also Cohen-Macaulay of relative dimension $r$ ) immediately gives us a (unique) map

$$
\theta_{g}^{f}: g^{\prime *} \omega_{f} \longrightarrow \omega_{f^{\prime}}
$$

such that $\int_{f^{\prime}} \circ R^{r} f_{*}^{\prime}\left(\theta_{g}^{f}\right)=g^{\#} \int_{f}$.

Remark 1.4.1. If $g$ is flat and $\Theta_{g}^{f}: g^{\prime *} f^{!} \stackrel{\sim}{\longrightarrow} f^{\prime !} g^{*}$ is the isomorphism defined in (1.6) (see Remark 1.3.2), then one checks easily that $H^{-r}\left(\Theta_{g}^{f}\left(\mathcal{O}_{Y}\right)\right)=\theta_{g}^{f}$.

Roughly speaking, Conrad's main results are as follows:

i) $\theta_{g}^{f}$ is an isomorphism.

ii) If $f$ is smooth, so that (via Verdier's identification [Ver69, p. 397, Theorem 3]) $\omega_{f}=\Omega_{f}^{r}$, $\omega_{f^{\prime}}=\Omega_{f^{\prime}}^{r}$, then $\theta_{g}^{f}$ is the canonical identification of differential forms $g^{\prime *} \Omega_{f}^{r}=\Omega_{f^{\prime}}^{r}$. Here $\Omega_{f}^{r}$ and $\Omega_{f^{\prime}}^{r}$ are the respective relative Kähler $r$-forms on $X$ and $X^{\prime}$.

Our main task in the proper case is to prove results i and ii based on our functorial foundations.

The above rough description involves some loss of the general flavor of [Con00]. Conrad begins with an explicit local description of $\omega_{f}$ and $\omega_{f^{\prime}}$ in terms of $\mathcal{E} x t$ sheaves. A little more precisely, if $X$ is a closed subscheme of a smooth $Y$-scheme $p: P \rightarrow Y$ (locally on $X$ such an embedding is possible), then $\omega_{f}$ can be identified with $\tilde{\omega}_{f}:=\mathcal{E}_{x} t_{\mathcal{O}_{P}}^{d}\left(\mathcal{O}_{X}, \Omega_{p}^{r+d}\right)$, where $d$ is the codimension of $X$ in $P$ (see $\S 8.1$ ). A similar description applies to $\omega_{f^{\prime}}$ in terms of $P^{\prime}=P \times_{Y} Y^{\prime}$. One has then, for these explicit versions of the dualizing sheaves, an explicit isomorphism (see (8.9) in $\S 8.1$ ) $\psi: g^{*} \tilde{\omega}_{f} \stackrel{\sim}{\longrightarrow} \tilde{\omega}_{f^{\prime}}$ obtained naturally on $\mathcal{E} x t$ sheaves (see [Con00, pp. 164-165, Theorem 3.6.1]) and which is shown to be independent of the local factorization of $f$ into a closed immersion followed by 


\section{P. SASTRY}

a smooth map. Conrad also has in place canonical traces for $R^{r} f_{*} \omega_{f}$ and $R^{r} f^{\prime}{ }_{*} \omega_{f^{\prime}}$. (See the proof of [Con00, p. 173, Corollary 3.6.6].) He shows that his map $\psi$ is compatible with the canonical traces. In other words, the explicit isomorphism $\psi$ he obtains is what we have called $\theta_{g}^{f}$. (He calls it $\beta_{f, g}$.) In contrast, our definition of $\theta_{g}^{f}$ is global (a priori it requires $f$ to be proper), and by definition is compatible with traces. We have to show that it is an isomorphism (and in the smooth case is a specific isomorphism.)

Remark 1.4.2. We have quoted Lemma 1, p. 39 of [Lip79] for a proof of the fact that the relative dualizing complex is concentrated in one degree, and the corresponding homology is flat over the base. The same lemma also asserts that the $\omega_{f}$ is well behaved with respect to base change, but this assertion is not completely proved there. The proof given in [Lip79] shows that there are local isomorphisms between $g^{\prime *} \omega_{f}$ and $\omega_{f^{\prime}}$, but it is not clear that these isomorphisms patch. This will follow from Theorem 2.3.6. (See question d raised in $\S 1.5$ below.)

\subsection{The problem refined}

Coming back to our formulation of the problem, a natural question is how necessary is the hypothesis of properness for our result? Note that the very definition of $\theta_{g}^{f}$ needs $f$ to be proper. Suppose we drop the properness assumption of $f$. Then $f$ can (at least locally) be compactified by $\bar{X} \stackrel{\bar{f}}{\longrightarrow} Y$ whose fibers are of dimension at most $r$ (see $\S 2.2$ ). These compactifications need not be CohenMacaulay, but if we set $\omega_{\bar{f}}=H^{-r}\left(\bar{f}^{!} \mathcal{O}_{Y}\right)$, we have a functorial isomorphism $\operatorname{Hom}_{\mathcal{O}_{X}}\left(\mathcal{F}, \omega_{\bar{f}}\right)=$ $\operatorname{Hom}_{\mathcal{O}_{Y}}\left(R^{r} \bar{f}_{*} \mathcal{F}, \mathcal{O}_{Y}\right)$ for $\mathcal{F} \in X_{\mathrm{qc}}$, whence an 'integral' $\int_{\bar{f}}: R^{r} \bar{f}_{*} \omega_{\bar{f}} \rightarrow \mathcal{O}_{Y}$. Arguing as before, we get a map $\theta_{g}^{\bar{f}}: \bar{g}^{*} \omega_{\bar{f}} \rightarrow \omega_{\overline{f^{\prime}}}$, where $\bar{f}^{\prime}: \bar{X}^{\prime} \rightarrow Y^{\prime}$ is the base change of $f$ under $g$, and $\bar{g}: \bar{X}^{\prime} \rightarrow \bar{X}$ the base change of $g$ under $\bar{f}$. The map $\theta_{g}^{\bar{f}}$ need not be an isomorphism (see [HS93, p. 773, Remark 3.4]), but (in light of Conrad's local and explicit results) we ask the following:

a) Is $\theta_{g}^{f}:=\theta_{g}^{\bar{f}} \mid X^{\prime}: g^{*} \omega_{f} \rightarrow \omega_{f^{\prime}}$ an isomorphism?

b) Is $\theta_{g}^{f}$ independent of the compactification of $\bar{f}$ ?

c) If $f$ is smooth, is $\theta_{g}^{f}$ the canonical identification of differential forms?

d) Given a factorization of $f$ as a closed immersion followed by a smooth map, and if the answer to questions a and b is 'yes', is there an explicit description of $\theta_{g}^{f}$ along the lines of Conrad's map?

e) Finally, is it possible to make explicit the trace map $R^{r} f_{*} \Omega_{f}^{r} \rightarrow \mathcal{O}_{Y}$ associated to Verdier's isomorphism when $f$ is smooth and proper?

These then are the problems. In this paper, we answer the first four questions affirmatively without recourse to dualizing complexes, and remaining faithful to the DV viewpoint. As for question e, we plan to address it in a later paper using the results of this paper together with the explicit computations made by Lipman in the proof of his 'residue theorem for projective space' [Lip84, p. 75, Proposition (8.5)]. Using c we note that if $\left\{U_{\alpha} \stackrel{f_{\alpha}}{\longrightarrow} Y\right\}$ is an open cover of $X \stackrel{f}{\longrightarrow} Y$ such that each $f_{\alpha}$ has an $r$-compactification (see $\S 2.2$ below for the precise definition of $r$-compactification), the various $\theta_{g}^{f_{\alpha}}$ patch together on $X^{\prime}$ to give a global isomorphism $\theta_{g}^{f}: g^{*} \omega_{f} \stackrel{\sim}{\longrightarrow} \omega_{f^{\prime}}$, which is independent of the cover $\left\{U_{\alpha}\right\}$. On the smooth locus of $f$, this isomorphism will be shown to be the canonical identification of differential forms. We state our results precisely in Theorems 2.3.3, 2.3.5 and 2.3.6.

Our techniques are such that we do not need dualizing complexes or their Cousin versions residual complexes. In later work we hope to use the DV approach to rework the theory of residues of Kunz, Hübl and Lipman [Hub89, HK90a, HK90b, Lip84, Lip87]. 


\section{Base Change For COHEN-MaCAulay MaPs}

\section{The main results}

\subsection{Verdier's isomorphism}

Let $f: X \longrightarrow Y$ be a smooth separated map of finite type. Theorem 3 (p. 397) of [Ver69] gives an isomorphism

$$
f^{!} \mathcal{O}_{Y} \stackrel{\sim}{\longrightarrow} \Omega_{f}^{r}[r]
$$

for $f$ smooth. We give Verdier's proof in $\S$ 7.1. The theorem depends only upon his flat base change theorem [Ver69, Theorem 2]. In [Ver69] the flat base change is only proved for schemes of finite Krull dimension. Fortunately, recent results of Lipman [Lip99, p. 120, Corollary (4.3)] yield the flat base change theorem (and even more), and hence (2.1), without the dimension hypotheses. One checks easily from the proof of [Ver69, Theorem 2] that the isomorphism (2.1) localizes well over open sets in $X$. This has implications when $f$ is just smooth and of finite type (not necessarily separated).

\subsection{Equidimensional maps}

In order to define $\theta_{g}^{f}$ for a Cohen-Macaulay map $f$ that is not proper, it becomes necessary to consider local compactifications of $f$ by maps whose fiber dimensions never exceed the relative dimension of $f$ (see $\S 2.3$ ). It is possible to get such local compactifications because $f$ is equidimensional. We now recall the notion of an equidimensional map as discussed in [GD64, 13.3] (see [GD64, (13.3.2)]). We restrict ourselves to finite type maps and retain our Noetherian hypothesis (though the notion of an equidimensional map is defined in greater generality in [GD64]). A finite type scheme map $f: X \rightarrow Y$ is said to be equidimensional if $f$ takes each maximal point of $X$ (i.e. a generic point of an irreducible component of $X$ ) to a maximal point of $Y$, and if there exists an integer $r$ such that every component of every non-empty fiber of $f$ has dimension $r$. Such an $r$ is called the relative dimension of $f$. We often abbreviate ' $f$ is equidimensional of relative dimension $r$ ' to ' $f$ is equidimensional of dimension $r$ '. A Cohen-Macaulay map of relative dimension $r$ (or any finite type flat map whose fibers have pure dimension $r$ ) is clearly equidimensional. Equidimensionality need not be preserved under base change (unless $f$ is flat). One could lose the dominance property, i.e. we could lose the property that generic points map to generic points. For example, set $X$ equal to an affine plane over a field, and set $Y$ equal to the union of this plane with a line $L$ meeting the plane in one point. Let $f: X \rightarrow Y$ be the inclusion map. Make the base change $g: L \rightarrow Y$ ( $g=$ inclusion map), and note that equidimensionality is lost. In order to have a notion which is compatible with base change, we make the following definition.

Definition 2.2.1. Let $r$ be a non-negative integer and $f: X \rightarrow Y$ a finite type map. The map $f$ is $r$-proper if it is proper and its fibers have dimension at most $r$. An $r$-compactification of $f$ is a compactification $\bar{f}: \bar{X} \rightarrow Y$ which is $r$-proper. The map $f$ is $r$-compactifiable if it has an $r$-compactification, and $f$ is locally $r$-compactifiable if $X$ can be covered by open sets each of which is $r$-compactifiable.

Note that the property of being $r$-proper (respectively $r$-compactifiable, respectively locally $r$-compactifiable) is preserved under base change.

Coming back to equidimensional maps, an equidimensional map $f: X \rightarrow Y$ of relative dimension $r$ is locally $r$-compactifiable. In greater detail, $f: X \rightarrow Y$ is equidimensional of dimension $r$ if and only if $X$ can be covered by open sets $U_{\alpha}$ such that the induced maps $f_{\alpha}: U_{\alpha} \rightarrow Y$ factor as $U_{\alpha} \stackrel{h_{\alpha}}{\longrightarrow} \mathbb{P}_{Y}^{r} \stackrel{\pi}{\longrightarrow} Y$ where $h_{\alpha}$ is equidimensional of dimension 0 (in particular quasi-finite) and $\pi$ is the usual projection (cf. [GD64, Proposition (13.3.1)]). By Zariski's Main Theorem, we have

a compactification $\bar{U}_{\alpha} \stackrel{\bar{h}_{\alpha}}{\longrightarrow} \mathbb{P}_{Y}^{r}$ of $h_{\alpha}$ such that $\bar{h}_{\alpha}$ is finite. The resulting map $\bar{f}_{\alpha}=\pi \circ \bar{h}_{\alpha}$ is an $r$ compactification of $f_{\alpha}$. In fact $\bar{f}_{\alpha}$ can be made equidimensional of dimension $r$, but its base changes need not be equidimensional of dimension $r$. 


\section{P. SASTRY}

\subsection{Kleiman's functor $f^{K}$}

Suppose a Cohen-Macaulay map $f$ has an $r$-compactification $\bar{f}$. To construct a comparison map $\theta_{g}^{f}$ for appropriate $g$ we extend the relative dualizing sheaf for $f$ to the compactification. There are many ways of doing this, but we choose a method involving the $r$-dualizing functors of Kleiman. The $r$-compactness of $\bar{f}$ and our choice of the extension of $\omega_{f}$ allow us to define a comparison map $\theta_{g}^{\bar{f}}$ associated with this extension. The map $\theta_{g}^{f}$ is then obtained by restricting. In greater detail, for a locally $r$-compactifiable map consider the variant $f^{K_{r}}=H^{-r}\left(f^{!}\right): Y_{\mathrm{qc}} \rightarrow X_{\mathrm{qc}}$ of Kleiman's $r$-dualizing functor (see [Kle80] for the definition of an $r$-dualizing functor). If the integer $r$ is clear from the context, and in most cases it will be, we will write $f^{K}$ for $f^{K_{r}}$.

Note. Since we are not assuming separatedness now, $f^{!}$does not make sense. However, its $-r$ th cohomology does make sense. To begin with, $X$ can be covered by open subschemes on which $f$ ! is defined. Over triple intersections, these objects formally satisfy cocycle rules. But that is not enough to glue them together as objects in $D_{\mathrm{qc}}^{+}(X)$ (the reason why Hartshorne upgrades his constructions to Cousin complexes). However, the $-r$ th cohomology does glue together since we are now in the category of sheaves! This is the slick way of understanding [HS93, p. 760, Corollary 1.7].

Remark 2.3.1. In view of the remarks made towards the end of $\S 2.1$, it is clear that if $f$ is smooth and not necessarily separated, we have an isomorphism

$$
f^{K} \mathcal{O}_{Y} \stackrel{\sim}{\longrightarrow} \Omega_{f}^{r}
$$

If $f$ is $r$-proper we claim that $f^{K}$ is isomorphic to Kleiman's $r$-dualizing functor. To see this, first note that under our hypotheses $R^{s} f_{*}=0$ for $s>r$, and from this one can (using descending induction on $k$ ) deduce that $H^{-k}\left(f^{!} \mathcal{G}\right)=0$ for $k>r, \mathcal{G} \in Y_{\mathrm{qc}} \subset D_{\mathrm{qc}}^{+}(Y)$. Therefore we have a bifunctorial isomorphism (from the adjoint relationship between $f^{!}$and $R f_{*}$ )

$$
\operatorname{Hom}_{\mathcal{O}_{X}}\left(\mathcal{F}, f^{K} \mathcal{G}\right) \stackrel{\sim}{\longrightarrow} \operatorname{Hom}_{\mathcal{O}_{Y}}\left(R^{r} f_{*} \mathcal{F}, \mathcal{G}\right)
$$

for $\mathcal{F} \in X_{\mathrm{qc}}$ and $\mathcal{G} \in Y_{\mathrm{qc}}$. The adjoint relationship between $f^{K}$ and $R^{r} f_{*}$ means that $f^{K}$ is an $r$-dualizing functor (by definition). Note that this adjoint relationship immediately gives rise to an $\mathcal{O}_{Y \text {-linear integral }}$

$$
\int_{f}^{\mathcal{G}}: R^{r} f_{*} f^{K} \mathcal{G} \longrightarrow \mathcal{G} .
$$

The pair $\left(f^{K} \mathcal{G}, \int_{f}^{\mathcal{G}}\right)$ is unique up to unique isomorphism.

Note that $(\cdot)^{K}$ is local (in the same sense that $(\cdot)^{!}$is local, see Remark 1.3.1). In fact the local property of $(\cdot)^{K}$ is defined via the local property of $(\cdot)^{!}$. If $\imath: U \rightarrow X$ is a open immersion, and $f_{U}: U \rightarrow Y$ the map induced by the $f: X \rightarrow Y$ as above, then

$$
\beta_{\imath}=\beta_{\imath}(f): \imath^{*} f^{K} \stackrel{\sim}{\longrightarrow} f_{U}^{K}
$$

will denote the resulting functorial isomorphism.

Remark 2.3.2. Let $\mathcal{G} \in Y_{\mathrm{qc}}$ and let $f$ be as above. Suppose $f$ proper. Since the complex $f^{!} \mathcal{G}$ has no cohomology below degree $-r$, we have a natural map $\kappa_{\mathcal{G}}: f^{K} \mathcal{G}[r] \longrightarrow f^{!} \mathcal{G}$ in $D_{\mathrm{qc}}^{+}(X)$. Since $R^{k} f_{*}\left(f^{K} \mathcal{G}[r]\right)=0$ for $k>0$, we get a map $\kappa_{\mathcal{G}}^{\prime}: R f_{*} f^{K} \mathcal{G}[r] \rightarrow H^{0}\left(R f_{*}\left(f^{K} \mathcal{G}\right)[r]\right)=R^{r} f_{*} f^{K} \mathcal{G}($ see (1.3)). One checks easily that

$$
T_{f}(\mathcal{G}) \circ R f_{*}\left(\kappa_{\mathcal{G}}\right)=\int_{f}^{\mathcal{G}} \circ \kappa_{\mathcal{G}}^{\prime} .
$$

Set $\omega_{f}=f^{K} \mathcal{O}_{Y}$. For $f$ proper, if no confusion arises, we will write $\int_{f}$ for $\int_{f}^{\mathcal{O}_{Y}}$. The pair $\left(\omega_{f}, \int_{f}\right)$ 


\section{Base Change For COHEN-MACAUlay MAPS}

is called a dualizing pair for $f$. Now consider the cartesian diagram

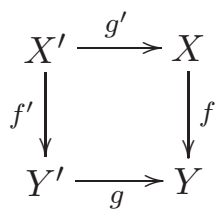

with (proper) $f$ as above. The canonical map $g^{*} R^{r} f_{*} \longrightarrow R^{r} f_{*}^{\prime} g^{\prime *}$ is an isomorphism (see Proposition B.1.2). Hence, as in the Cohen-Macaulay case, we have a map

$$
g^{\#} \int_{f}: R^{r} f_{*}^{\prime} g^{\prime *} \omega_{f} \longrightarrow \mathcal{O}_{Y^{\prime}}
$$

induced by $g^{*} \int_{f}$. As before we have a map

$$
\theta_{g}^{f}: g^{\prime *} \omega_{f} \longrightarrow \omega_{f^{\prime}}
$$

Our main theorem is as follows.

THEOREM 2.3.3. Let

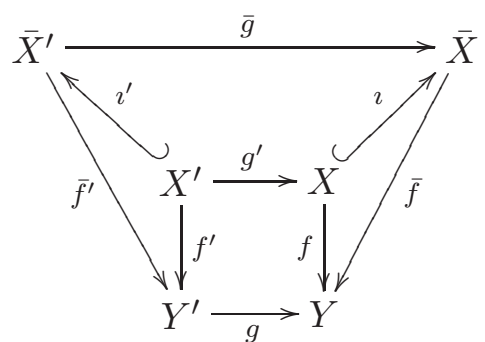

be a commutative diagram of schemes such that

- $f$ is Cohen-Macaulay of relative dimension $r$;

- $\imath$ is an open immersion;

- $\bar{f}$ is r-proper;

- the inner square, the outer trapezium, and the trapezium bordered by $g^{\prime}, \imath^{\prime}, \imath$ and $\bar{g}$ are all cartesian.

Then the following hold.

a) The map $\theta_{g}^{\bar{f}} \mid X^{\prime}: g^{\prime *} \omega_{f} \rightarrow \omega_{f^{\prime}}$ is independent of the r-compactification $\bar{f}$ of $f$. Call the map $\theta_{g}^{f}$.

b) $\theta_{g}^{f}$ is an isomorphism.

c) If $f$ is smooth, and we identify $\omega_{f}, \omega_{f^{\prime}}$ respectively with $\Omega_{f}^{r}, \Omega_{f^{\prime}}^{r}$, via Verdier's isomorphism (2.1) (or (2.2)), then $\theta_{g}^{f}$ is the canonical identification of differential forms $g^{\prime *} \Omega_{f}^{r}=\Omega_{f^{\prime}}^{r}$.

Explanation. Part a needs slight elaboration. Suppose $\bar{f}_{j}: \bar{X}_{j} \rightarrow Y, j=1,2$, are two $r$-compactifications of $f$, with $\imath_{j}: X \rightarrow \bar{X}_{j}$ the corresponding open immersion. Suppose (in an obvious notation) $\beta_{j}: \omega_{f} \rightarrow \imath_{j}{ }^{*} \omega_{\bar{f}_{j}}$ and $\beta_{j}^{\prime}: \omega_{f^{\prime}} \rightarrow \imath_{j}^{\prime *} \omega_{\bar{f}_{j}^{\prime}}, j=1,2$, are the resulting isomorphisms (see (2.3)). Then part a asserts that

$$
\beta_{1}^{\prime-1} \circ \imath_{1}^{\prime *} \theta_{g}^{\bar{f}_{1}} \circ g^{\prime *} \beta_{1}=\beta_{2}^{\prime-1} \circ \imath_{2}^{\prime *} \theta_{g}^{\bar{f}_{2}} \circ g^{\prime *} \beta_{2}
$$

Remark 2.3.4. Assume $g$ is flat. Let $\Theta_{g}^{\bar{f}}$ be as in Remark 1.3.2 (see (1.6)). One checks that $\theta_{g}^{\bar{f}}=$ $H^{-r}\left(\Theta_{g}^{\bar{f}}\left(\mathcal{O}_{Y}\right)\right)$. If $\bar{f}=f$ then we recover Remark 1.4.1. Moreover, diagram (1.11) shows that the 


\section{P. SASTRY}

map $\theta_{g}^{\bar{f}} \mid X^{\prime}$ (which equals $H^{-r}\left(\imath^{\prime *} \Theta_{g}^{\bar{f}}\left(\mathcal{O}_{Y}\right)\right)$ ) is independent of the $r$-compactification $\bar{f}$ of $f$ (in the sense of the Explanation above). Since $\Theta_{g}^{\bar{f}}$ is an isomorphism, therefore so is $\theta_{g}^{\bar{f}} \mid X^{\prime}$. Thus parts a and b of Theorem 2.3.3 are true for $g$ flat. Incidentally, if $g$ is flat, $\theta_{g}^{f}$ can be defined even if the compactification $\bar{f}$ is not r-proper. Indeed the map $H^{-r}\left(l^{\prime *} \Theta_{g}^{\bar{f}}\left(\mathcal{O}_{Y}\right)\right)$ is independent of the compactification $\bar{f}$ in the sense of the Explanation above with $\omega_{\bar{f}_{j}}$ and $\omega_{\bar{f}_{j}^{\prime}}$ being replaced by $H^{-r}\left(\bar{f}_{j}^{!} \mathcal{O}_{Y}\right)$ and $H^{-r}\left(\bar{f}^{\prime}{ }_{j} \mathcal{O}_{Y^{\prime}}\right)$ respectively (cf. (1.11)). Clearly $\theta_{g}^{f}$ so defined is an isomorphism.

Now suppose $f: X \rightarrow Y$ is Cohen-Macaulay of relative dimension $r$ (not necessarily separated) and consider the base change diagram as follows.

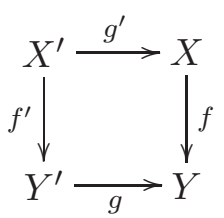

Since $f$ is equidimensional of dimension $r$, it is locally $r$-compactifiable. In other words, $X$ can be covered by open subsets $\left\{U_{\alpha}\right\}$ such that each map $f_{\alpha}:=f \mid U_{\alpha}: U_{\alpha} \rightarrow Y$ has an $r$-compactification $\bar{f}_{\alpha}$. By part a of the previous theorem, the maps $\theta_{g}^{f_{\alpha}}$ glue together to give a global $\mathcal{O}_{X^{\prime}}$-map

$$
\theta_{g}^{f}: g^{\prime *} \omega_{f} \longrightarrow \omega_{f^{\prime}}
$$

This map (again from part a of Theorem 2.3.3) is independent of the cover $\left\{U_{\alpha}\right\}$. Part b of the theorem then implies that $\theta_{g}^{f}$ is an isomorphism. Therefore Theorem 2.3.3 has the following, seemingly more general, reformulation.

THEOREM 2.3.5. Let

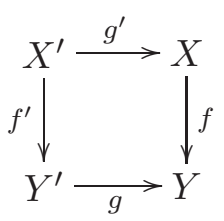

be a cartesian square of schemes, with $f$ Cohen-Macaulay of relative dimension $r$. Then the following hold.

a) There exists an isomorphism

$$
\theta_{g}^{f}: g^{\prime *} \omega_{f} \stackrel{\sim}{\longrightarrow} \omega_{f^{\prime}}
$$

characterized by the property that if $U \subset X$ is an open set admitting an $r$-compactification over $Y$ and $\theta_{g}^{f \mid U}$ is the map in Theorem 2.3.3, part a, then

$$
\theta_{g}^{f} \mid g^{\prime-1}(U)=\theta_{g}^{f \mid U}
$$

b) If $f$ is smooth and $\omega_{f}, \omega_{f^{\prime}}$ are identified with $\Omega_{f}^{r}, \Omega_{f^{\prime}}^{r}$ via $(2.2)$, then $\theta_{g}^{f}$ is the canonical identification of differential forms $g^{\prime *} \Omega_{f}^{r}=\Omega_{f^{\prime}}^{r}$.

Our final result is concerned with question d raised in $\S 1.5$. (See the discussion on Conrad's work at the end of $\S$ 1.4.) To that end, suppose $f: X \rightarrow Y$ is Cohen-Macaulay of relative dimension $r$ and $f$ factors as $X \stackrel{\imath}{\rightarrow} P \stackrel{p}{\rightarrow} Y$ where $\imath$ is a closed immersion and $p$ is a smooth map of relative 
dimension $r+d$. Let $g: Y^{\prime} \rightarrow Y$ be a map of schemes and

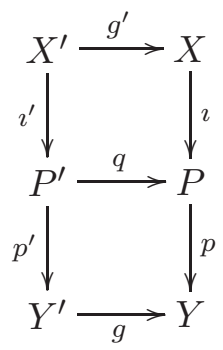

the resulting composite cartesian diagram. Let $\tilde{\omega}_{f}$ be the coherent $\mathcal{O}_{X}$-module associated to the $\imath_{*} \mathcal{O}_{X}$-module $\mathcal{E x t}_{\mathcal{O}_{P}}^{d}\left(\imath_{*} \mathcal{O}_{X}, \Omega_{P / Y}^{r+d}\right)$ and $\tilde{\omega}_{f^{\prime}}$ the coherent $\mathcal{O}_{X^{\prime}}$-module associated to $\mathcal{E} t_{\mathcal{O}_{P^{\prime}}}^{d}\left(\imath^{\prime}{ }_{*} \mathcal{O}_{X^{\prime}}\right.$, $\left.\Omega_{P^{\prime} / Y^{\prime}}^{r+d}\right)$. It is well known (adjunction) that $\tilde{\omega}_{f}$ and $\tilde{\omega}_{f^{\prime}}$ are explicit representations of $\omega_{f}$ and $\omega_{f^{\prime}}$. In $\S 8.1$ we spell out these identifications. Next, there is an obvious isomorphism between $g^{\prime *} \tilde{\omega}_{f}$ and $\tilde{\omega}_{f^{\prime}}$. One wants to know if this isomorphism is $\theta_{g}^{f}$. In greater detail let $\varphi: \omega_{f} \stackrel{\sim}{\longrightarrow} \tilde{\omega}_{f}$ and $\varphi^{\prime}: \omega_{f^{\prime}} \stackrel{\sim}{\longrightarrow} \tilde{\omega}_{f^{\prime}}$ be the isomorphisms described in (8.4) and (8.7) and $\psi_{g}^{f}: g^{\prime *} \tilde{\omega}_{f} \stackrel{\sim}{\longrightarrow} \tilde{\omega}_{f^{\prime}}$ the isomorphism (8.9). Our third main result, to be proven in $\S 8$, is as follows (cf. [Con00, pp. 164-165, Theorem 3.6.1]).

Theorem 2.3.6. The following diagram commutes.

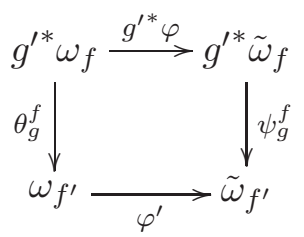

Remark 2.3.7. If $d=0$ in the above theorem, then we recover part b of Theorem 2.3.5.

Remark 2.3.8. We would like flexibility in choosing dualizing pairs when proving our theorems. To this end let $f: X \rightarrow Y$ be $r$-proper and consider the base change diagram (2.4). Suppose $\left(\tilde{\omega}_{f}, \tilde{\int}_{f}\right)$ (respectively $\left(\tilde{\omega}_{f^{\prime}}, \tilde{\int}_{f^{\prime}}\right)$ ) is another $r$-dualizing pair for $f$ (respectively $f^{\prime}$ ). Let $\tilde{\theta}_{g}^{f}: g^{\prime *} \tilde{\omega}_{f} \rightarrow \tilde{\omega}_{f^{\prime}}$ be the map defined in the way that $\theta_{g}^{f}$ was defined in (2.5). Then

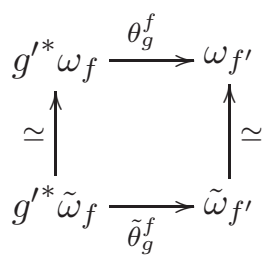

commutes, where the vertical isomorphisms arise from uniqueness (up to unique isomorphism) of dualizing pairs. Indeed, if $\eta: \tilde{\omega}_{f} \rightarrow \omega_{f}$ and $\eta^{\prime}: \tilde{\omega}_{f^{\prime}} \rightarrow \omega_{f^{\prime}}$ are these unique isomorphisms, then

$$
\begin{aligned}
\int_{f^{\prime}} \circ R^{r} f_{*}^{\prime}\left(\theta_{g}^{f} \circ g^{\prime *} \eta\right) & =\int_{f^{\prime}} \circ R^{r} f_{*}^{\prime}\left(\theta_{g}^{f}\right) \circ R^{r} f_{*}^{\prime}\left(g^{\prime *} \eta\right) \\
& =g^{\#} \int_{f} \circ R^{r} f_{*}^{\prime}\left(g^{\prime *} \eta\right) \\
& =g^{\#} \int_{f}^{\tilde{r}}
\end{aligned}
$$




\section{P. SASTRY}

and

$$
\begin{aligned}
\int_{f^{\prime}} \circ R^{r} f_{*}^{\prime}\left(\eta^{\prime} \circ \tilde{\theta}_{g}^{f}\right) & =\int_{f^{\prime}} \circ R^{r} f_{*}^{\prime}\left(\eta^{\prime}\right) \circ R^{r} f_{*}^{\prime}\left(\tilde{\theta}_{g}^{f}\right) \\
& =\int_{f^{\prime}} \circ R^{r} f_{*}^{\prime}\left(\tilde{\theta}_{g}^{f}\right) \\
& =g^{\#} \int_{f} .
\end{aligned}
$$

Thus by the universal property of $\left(\omega_{f^{\prime}}, \int_{f^{\prime}}\right), \eta^{\prime} \circ \tilde{\theta}_{g}^{f}=\theta_{g}^{f} \circ g^{\prime *} \eta$. Note, in particular, that $\theta_{g}^{f}$ is an isomorphism if and only if $\tilde{\theta}_{g}^{f}$ is.

\section{Main ideas}

The key idea is this: we define a residue map $\operatorname{res}_{Z}: R_{Z}^{r} f_{*} \omega_{f} \rightarrow \mathcal{O}_{Y}$ for appropriate closed subschemes $Z$ of $X$. The residue map is a formal analog ${ }^{1}$ of the integral $\int_{f}$. The idea of using residues and so working with formal schemes (in perhaps a disguised way) has many precedents and we mention [Ver69, Lip84, HK90a, HK90b, HS93, LS92, Con00] in passing. In this paper, we will show, in a fairly elementary way, that for special $Z$ (we call such $Z$ 's good) res $Z$ has a local duality property and is well behaved with respect to base change. Recall that if $Z \stackrel{J}{\hookrightarrow} X$ is a closed subscheme of $X$, then $R_{Z}^{p} f_{*}$ denotes the $p$ th right derived functor of $f_{*} \underline{\Gamma}_{Z}$, where $\underline{\Gamma}_{Z}$ is the sheafified version of 'sections with support in $Z$ '. The corresponding derived functor $D_{\mathrm{qc}}^{+}(X) \rightarrow D_{\mathrm{qc}}^{+}(Y)$ is denoted $R_{Z} f_{*}$.

\subsection{Residues}

Let $f: X \rightarrow Y$ be a separated Cohen-Macaulay map of relative dimension $r, Z \stackrel{\jmath}{\hookrightarrow} X$ a closed immersion such that $h=j \circ f: Z \rightarrow Y$ is proper. Suppose

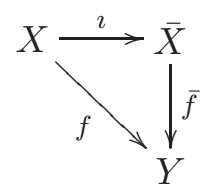

is a compactification of $f$ (not necessarily $r$-proper). In $D_{\mathrm{qc}}^{+}$we have a sequence of maps (the second one arising from excision)

$$
R_{Z} f_{*} \omega_{f}[r] \stackrel{\sim}{\longrightarrow} R_{Z} f_{*} v^{*} \bar{f}^{!} \mathcal{O}_{Y} \stackrel{\sim}{\longrightarrow} R_{Z} \bar{f}_{*} \bar{f}^{!} \mathcal{O}_{Y} \longrightarrow R \bar{f}_{*} \bar{f}^{!} \mathcal{O}_{Y} \stackrel{T_{\bar{f}}}{\longrightarrow} \mathcal{O}_{Y} .
$$

Taking the 0th cohomology of the above composite (and using $(1.3)$ ) we get the $\left(\mathcal{O}_{Y}\right.$-linear) residue map:

$$
\operatorname{res}_{Z}: R_{Z}^{r} f_{*} \omega_{f} \longrightarrow \mathcal{O}_{Y}
$$

Proposition 3.1.1. The residue map $\operatorname{res}_{Z}: R_{Z}^{r} f_{*} \omega_{f} \rightarrow \mathcal{O}_{Y}$ does not depend on the compactification $(\imath, \bar{f})$ of $f$.

Proof. Let $\left(\imath_{k}, f_{k}: X_{k} \rightarrow Y\right), k=1,2$, be two compactifications of $f$. By taking the closure of the diagonal embedding of $X$ in $X_{1} \times_{Y} X_{2}$ if necessary, we may assume that we have a commutative

\footnotetext{
${ }^{1}$ This philosophy is made precise in [AJL99]. However, we will not appeal to that work for residues.
} 
diagram

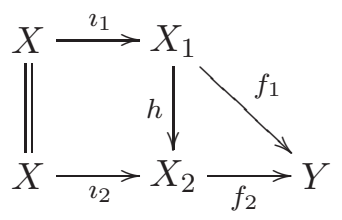

with the square being cartesian. The proposition follows from the commutativity of

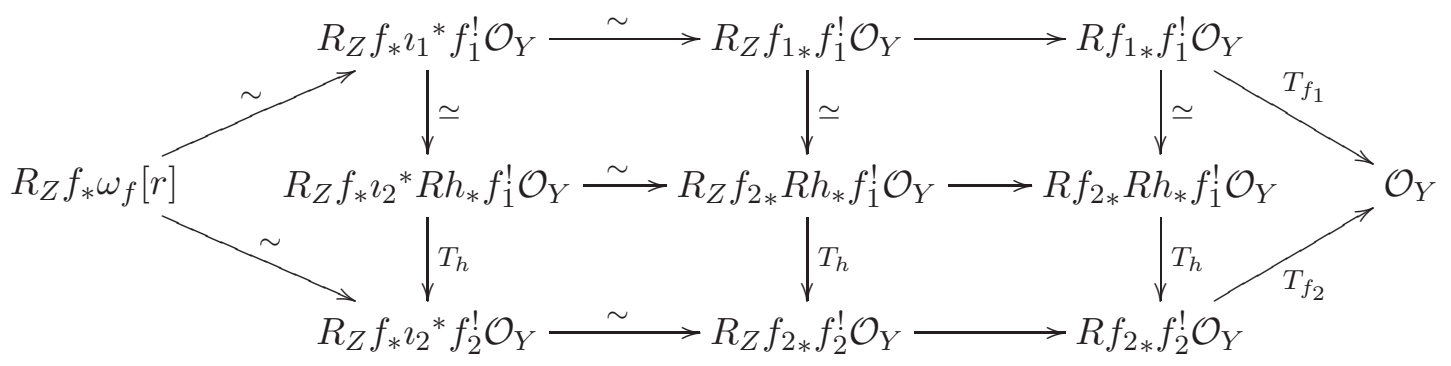

We point out that the triangle on the left commutes since it does so before applying the functor $R_{Z} f_{*}$ (see Remark 1.3.1, especially the isomorphism (1.8)).

Remark 3.1.2. If $(\imath, \bar{f}: \bar{X} \rightarrow Y)$ is an $r$-compactification of $f$ (so that $\bar{f}^{K}: Y_{\mathrm{qc}} \rightarrow \bar{X}_{\mathrm{qc}}$ is defined), then with $\omega_{\bar{f}}=\bar{f}^{K} \mathcal{O}_{Y}$ we see easily that $\operatorname{res}_{Z}$ can be defined by the commutativity of the following diagram.

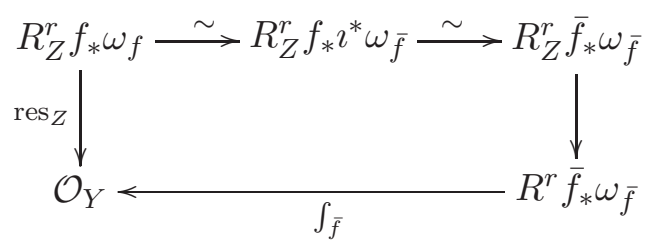

The following result is a way of saying that residues behave well with respect to certain base changes.

Proposition 3.1.3. Let

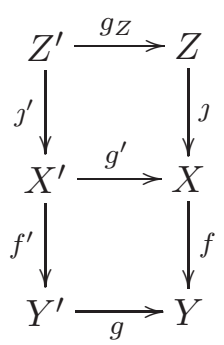

be a commutative diagram of cartesian squares with $f$ a separated Cohen-Macaulay map of relative dimension $r$ and $\jmath: Z \hookrightarrow X$ a closed immersion such that $Z$ is proper over $Y$. Suppose $(X \stackrel{\imath}{\hookrightarrow}$ $\bar{X}, \bar{X} \stackrel{\bar{f}}{\longrightarrow} Y)$ is a compactification of $f$ and $\left(X^{\prime} \stackrel{\imath^{\prime}}{\hookrightarrow} \bar{X}^{\prime}, \bar{X}^{\prime} \stackrel{\bar{f}^{\prime}}{\longrightarrow} Y^{\prime}\right)$ its base change by $g: Y \rightarrow Y^{\prime}$. Let $\bar{g}: \bar{X}^{\prime} \rightarrow \bar{X}$ be the resulting projection map. Assume that one of the following conditions holds:

a) The map $\bar{f}$ is r-compact.

b) The map $g$ is flat. 


\section{P. SASTRY}

Then the diagram

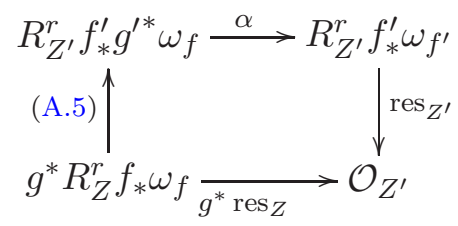

commutes where $\alpha=\imath^{\prime *} \theta_{g}^{\bar{f}}$ in case a and $\alpha=\theta_{g}^{f}$ in case b.

Proof. Case a is dealt with as follows. Consider the diagram below.

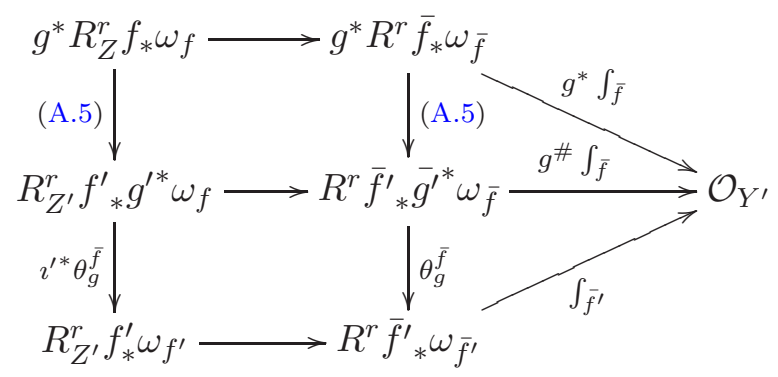

The top subrectangle commutes by Proposition A.1.1, part a. The remaining subdiagrams commute by the definitions of $g^{\#} \int_{\bar{f}}, \theta_{g}^{\bar{f}}$, and the functoriality of the map $R_{Z^{\prime}}^{r} f_{*}^{\prime} g^{\prime *} \rightarrow R^{r} \bar{f}^{\prime}{ }_{*} \bar{g}^{\prime *}$.

Case b is proved in essentially the same way. First apply $L g^{*}$ to the composite in (3.1) and use Remark B.2.2 along with the map $\Theta_{g}^{f}$ (see (1.6) and Remark 1.3.2) to compare with the composite analogous to $(3.1)$ for the triple $\left(Z^{\prime}, f^{\prime}, \bar{f}^{\prime}\right)$.

We are not interested in arbitrary $Z$. Our interest is in 'good' immersions, which we define in a moment. Recall that a sequence $\boldsymbol{t}=\left(t_{1}, \ldots, t_{r}\right)$ in a ring $R$ is said to be Koszul regular if the Koszul homology complex built from $\boldsymbol{t}$ resolves $R / \boldsymbol{t} R$.

Definition 3.1.4. Let $f, Z$ be as at the start of $\S 3.1$. The closed immersion $\jmath: Z \hookrightarrow X$ is said to be good if it satisfies the following hypotheses (cf. also [HK90a, (4.3)]):

- There is an affine open covering $\mathcal{U}=\left\{U_{\alpha}=\operatorname{Spec} A_{\alpha}\right\}$ of $Y$, and for each index $\alpha$ there is an affine open subscheme $V_{\alpha}=\operatorname{Spec} R_{\alpha}$ of $f^{-1}\left(U_{\alpha}\right)$ such that $Z \cap f^{-1}\left(U_{\alpha}\right) \subset V_{\alpha}$.

- The closed immersion $\jmath$ is given in $V_{\alpha}$ by a Koszul-regular $R_{\alpha}$-sequence.

Note that since $Z$ is proper and affine over $Y$, therefore $h=f \circ \jmath: Z \rightarrow Y$ is finite.

Lemma 3.1.5. Let $f: X \rightarrow Y$ be Cohen-Macaulay of relative dimension $r$, and $\jmath: Z \hookrightarrow X$ a good immersion with respect to $f$.

a) [GD64, Chapitre 0, 15.1.16] The map $h=f \circ \jmath: Z \rightarrow Y$ is flat.

b) If

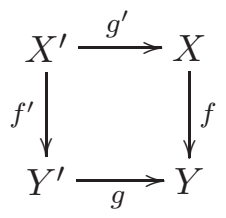

is a cartesian square then $\jmath^{\prime}: Z^{\prime}=g^{-1}(Z) \hookrightarrow X^{\prime}$ is a good immersion with respect to $f^{\prime}$.

Proof. Part a is proved in the indicated reference in [GD64] and also in [Mat86, p. 177, Corollary to 22.5 and Theorem 22.6]. Part b follows from the discussion in [GD64, Chapitre 4, $\S 19.2$ ], but the proof is simple enough (in our situation) for us to give it again. Our definition of good immersion 


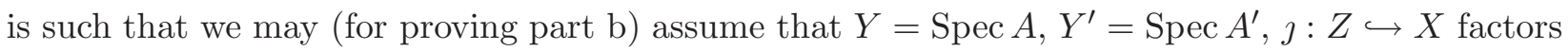
through an affine open set $U=\operatorname{Spec} R$ of $X$, and $Z$ is defined by an ideal $I$ of $R$ generated by a Koszul-regular $R$-sequence $\boldsymbol{t}=\left(t_{1}, \ldots, t_{r}\right)$. Since $R$ and $B=R / I$ are flat over $A$, the extension of $\boldsymbol{t}$ to $R^{\prime}=R \otimes_{A} A^{\prime}$ is Koszul-regular (indeed the Koszul complex on $\boldsymbol{t}$ augmented by $B$ on the right is an exact complex of flat $A$ modules, and hence retains its exactness on tensoring with $A^{\prime}$ over $A$ ). This proves that $\jmath^{\prime}: Z^{\prime} \hookrightarrow X^{\prime}$ is a good immersion for $f^{\prime}$.

\subsection{Two key propositions}

For proving Theorem 2.3.3, parts a and b, the crucial ingredients are (a) local duality (Proposition 3.2.1 below) and (b) compatibility of local duality with base change (Proposition 3.2.2 below). The proofs of these two propositions will be given later, after we show (in $\S 3.3$ ) how a substantial part of the main result, Theorem 2.3.3, is proved using these propositions.

For good immersions $Z \stackrel{\jmath}{\hookrightarrow} X$ with respect to a separated Cohen-Macaulay map $f: X \rightarrow Y$ we have a version of local duality. To fix ideas we will first describe it in the 'affine situation', i.e. $Y=\operatorname{Spec} A, X=\operatorname{Spec} R, Z=\operatorname{Spec} B$, and $B=R / I$, where $I$ is generated by a Koszulregular $R$-sequence $t_{1}, \ldots, t_{r}$. Let $\omega_{R / A}=\Gamma\left(X, \omega_{f}\right)$ and for any $R$-module $N$ let $\widehat{N}$ denote the completion of $M$ with respect to the ideal $I$ of $R$. For a finitely generated $\widehat{R}$-module $M$, set $D_{I}(M)=$ $\operatorname{Hom}_{A}\left(H_{\widehat{I}}^{r}(M), A\right)$. Note that $D_{I}$ is a contravariant functor. Now set $\operatorname{res}_{I}=\Gamma(Y, \operatorname{res} Z): H_{I}^{r}\left(\omega_{R / A}\right) \rightarrow$ A. By Remarks C.2.2 and C.4.2, $\operatorname{res}_{I} \in D_{I}\left(\widehat{\omega}_{R / A}\right)$. Our local duality statement is that the functor $D_{I}$ is represented by the pair $\left(\widehat{\omega}_{R / A}, \operatorname{res}_{I}\right)$. We can upgrade this to the general situation, where we no longer assume that $X, Y$ and $Z$ are affine. More precisely, let $\jmath: Z \rightarrow X$ be a good immersion with respect to the Cohen-Macaulay map $f$. Let $\widehat{X}$ be the formal scheme obtained by completing $X$ along $Z$, and $\widehat{f}: \widehat{X} \rightarrow Y$ the resulting morphism. Let $\widehat{X}_{c}$ denote the category of coherent $\mathcal{O}_{\widehat{X}}$-modules. For any coherent sheaf $\mathcal{F}$ defined in an open neighborhood of $Z$ in $X$, let $\widehat{\mathcal{F}}_{Z}$ denote the completion of $\mathcal{F}$ along $Z$. Since $Z \hookrightarrow X$ is a good immersion for $f$, we can define a functor $R_{Z}^{r} \widehat{f}_{*}: \widehat{X}_{c} \rightarrow Y_{\mathrm{qc}}$ in such a way that $R_{Z}^{r} \widehat{f}_{*}\left(\widehat{\mathcal{F}}_{Z}\right)=R_{Z}^{r} f_{*}(\mathcal{F})$ for $\mathcal{F}$ in $X_{c}$. In greater detail suppose $\mathcal{G} \in \widehat{X}_{c}$. Let $U_{\alpha}=\operatorname{Spec} A$ be a member of the affine open covering $\mathcal{U}$ of $Y$ required in the definition of good immersion (3.1.4). Let $V_{\alpha}=\operatorname{Spec} R \subset f^{-1}\left(U_{\alpha}\right)$ be as in Definition 3.1.4 and let $I \subset R$ be the ideal corresponding to the closed subscheme $Z \cap f^{-1}\left(U_{\alpha}\right)$ of $V_{\alpha}$. If $\widehat{R}$ is the completion of $R$ in the $I$-adic topology, one checks that $\widehat{f}^{-1}\left(U_{\alpha}\right)=\operatorname{Spf}(\widehat{R})$ and that $\mathcal{G} \mid \widehat{f}^{-1}\left(U_{\alpha}\right)$ is the sheaf corresponding to a finitely generated $\widehat{R}$-module $N$ (cf. [Har77, Theorem (9.7), p. 198]). The $\widehat{R}$-module $H_{\widehat{I}}^{r}(N)$ is an $A$-module and one checks that it localizes well with respect to affine open localizations of $U_{\alpha}=\operatorname{Spec} A$. Varying $U_{\alpha}$ over the affine open cover $\mathcal{U}$ of $Y$, one checks that these modules glue and we get an $\mathcal{O}_{Y}$-module which we denote $R_{Z}^{r} \widehat{f}_{*} \mathcal{G}$. Note that if $\mathcal{F} \in X_{c}$ then $R_{Z}^{r} \widehat{f}_{*}\left(\widehat{\mathcal{F}}_{Z}\right)=R_{Z}^{r} f_{*}(\mathcal{F})$. In affine terms this corresponds to the equality $H_{I}^{r}(N)=H_{\widehat{I}}^{r}(\widehat{N})$ (see Remark C.2.2). Let $D_{Z}$ be the functor on $\widehat{X}_{c}$ given by $D_{Z}=\operatorname{Hom}_{\mathcal{O}_{Y}}\left(R_{Z}^{r} \widehat{f}_{*}(\cdot), \mathcal{O}_{Y}\right)$. Making the identification $R_{Z}^{r} \widehat{f}_{*}\left(\widehat{\omega}_{f}\right)_{Z}=R_{Z}^{r} f_{*} \omega_{f}$, we have the following local duality assertion (see $\S 5.2$ ).

Proposition 3.2.1 (Local duality). The pair $\left(\left(\widehat{\omega_{f}}\right)_{Z}, \operatorname{res}_{Z}\right)$ represents $D_{Z}$.

Next suppose

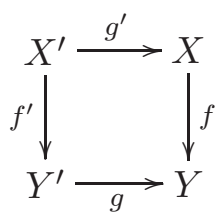

is a cartesian square ( $f$ as before, Cohen-Macaulay of relative dimension $r$ and separated). Suppose $\jmath: Z \hookrightarrow X$ is a good immersion for $f$. Let $\jmath^{\prime}: Z^{\prime} \hookrightarrow X^{\prime}$ and $g^{\prime \prime}: Z^{\prime} \rightarrow Z$ be the corresponding base 


\section{P. SASTRY}

change maps. Note that by Lemma 3.1.5 $\jmath^{\prime}: Z^{\prime} \hookrightarrow X^{\prime}$ is a good immersion for $f^{\prime}$. Define

$$
g^{\#} \operatorname{res}_{Z}: R_{Z^{\prime}}^{r} f_{*}^{\prime} g^{\prime *} \omega_{f} \longrightarrow \mathcal{O}_{Y}
$$

by the commutativity of the following diagram.

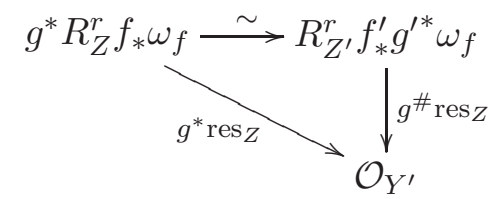

The horizontal map is the canonical base change map defined in (A.5) in the appendix which is an isomorphism (see Proposition B.1.3).

Our second key proposition is now given.

Proposition 3.2.2. The pair $\left(\left(\widehat{g^{\prime *} \omega_{f}}\right)_{Z^{\prime}}, g^{\#} \operatorname{res}_{Z}\right)$ represents $D_{Z^{\prime}}$.

\subsection{Proof of Theorem 2.3.3, parts a and $b$}

In this subsection we assume Propositions 3.2.1 and 3.2.2 whose proofs are given in $\S \S 5.2$ and 6.2 respectively. We first make a few remarks whose main aim is to show that the comparison map $\theta_{g}^{f}$ is compatible with Zariski localization on either base.

Remark 3.3.1. Consider the following diagram of cartesian squares with $f r$-proper.

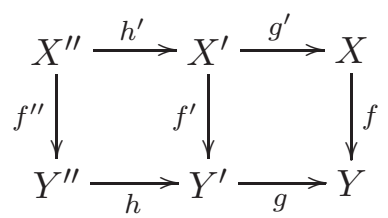

We can show that

$$
\theta_{g h}^{f}=\theta_{h}^{f^{\prime}} \circ h^{\prime *} \theta_{g}^{f} .
$$

The strategy is as follows. Let $\theta_{1}$ be the left side and $\theta_{2}$ be the right side. Then one checks (using the definition of the various $\theta^{\prime}$ s) that $\int_{f^{\prime \prime}} \circ R^{r} f_{*}^{\prime \prime}\left(\theta_{1}\right)=\int_{f^{\prime \prime}} \circ R^{r} f_{*}^{\prime \prime}\left(\theta_{2}\right)$. One needs the commutativity of the diagram

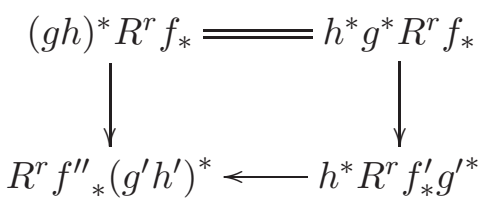

where all arrows arise from (A.5). One can prove this in the following (inelegant) way: the map arising from (A.5) is compatible with Zariski localization of either base, and so we may assume $Y$, $Y^{\prime}$ and $Y^{\prime \prime}$ are affine. Now use the commutative diagram (B.1). The more elegant approach is of course to prove that the diagram

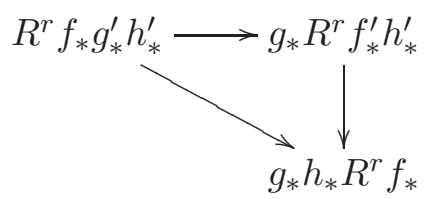

commutes. This follows from [Lip03, pp. 112-113, Proposition (3.7.2)(iii)]. 
Remark 3.3.2. Suppose

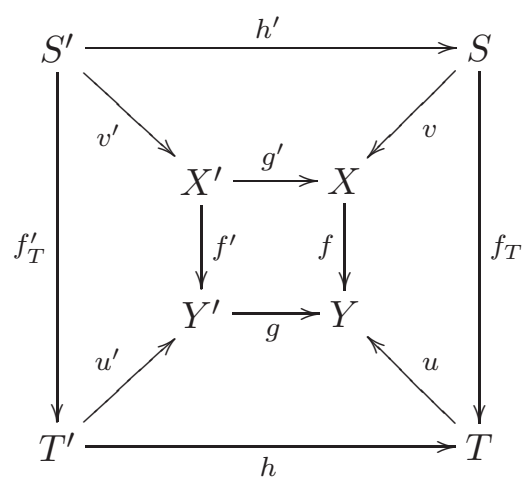

is a commutative diagram with $f$ locally $r$-compactifiable and such that the inner square, outer square, and the four trapeziums squeezed between them are all cartesian. Note that if the inner cartesian square is given, then the rest of the diagram is completely determined by the single map $u: T \rightarrow Y$. If $f$ is $r$-proper then from Remark 3.3.1 we easily see that the following diagram commutes

$$
\theta_{h}^{f_{T} \circ h^{\prime *}} \theta_{u}^{f}=\theta_{u^{\prime}}^{f^{\prime}} v^{\prime *} \theta_{g}^{f} .
$$

In fact both sides equal $\theta_{u h}^{f}=\theta_{g u^{\prime}}^{f}$. This theme recurs in different contexts in this paper (cf. also $\S 8.2$, and Remark B.2.3 and B.2.4).

Remark 3.3.3. In view of Remark 2.3.4, if $g: Y^{\prime} \rightarrow Y$ is an open immersion we can set $\omega_{f^{\prime}}=g^{\prime *} \omega_{f}$ and $\theta_{g}^{f}$ equal to the identity without affecting compatibilities. This coupled with Remark 3.3.2 means that for parts a and b of Theorem 2.3.3 the question is local on $Y$. Similarly, Remark 3.3.1 says that these questions are local on $Y^{\prime}$ also. We may sometimes implicitly make these reductions. In all such instances, this remark underlies these reductions.

Consider the situation in Theorem 2.3.3. We are about to prove parts a and $\mathrm{b}$ of the Theorem (assuming Propositions 3.2.1 and 3.2.2). Suppose first that we have a good immersion $\jmath: Z \hookrightarrow X$ for $f$ (a strong assumption, and in general there is no guarantee that such an immersion exists). Let $Z^{\prime}=g^{\prime-1}(Z)$. Using Proposition 3.1.3 and the definition of $g^{\#} \operatorname{res}_{Z}$ (cf. (3.4)) we have

$$
g^{\#} \operatorname{res}_{Z}=\operatorname{res}_{Z^{\prime}} \circ R_{Z^{\prime}}^{r} f_{*}^{\prime}\left(l^{\prime *} \theta_{g}^{\bar{f}}\right) .
$$

Since $\left(\widehat{g^{\prime *} \omega_{f, Z^{\prime}}}, g^{\#} \operatorname{res} Z\right)$ and $\left(\widehat{\omega_{f^{\prime}, Z^{\prime}}}\right.$, res $\left._{Z^{\prime}}\right)$ represent the same functor (here it is being used that we assume Propositions 3.2.1 and 3.2.2 are true), it follows that:

- with obvious notation $\widehat{\theta}_{g, Z^{\prime}}^{\bar{f}}$, does not depend on the compactification $\bar{f}$ indeed $\operatorname{res}_{Z}, g^{\#} \operatorname{res}_{Z}$, and $\operatorname{res}_{Z^{\prime}}$ are all independent of $\bar{f}$, giving the conclusion;

- $\widehat{\theta}_{g, Z^{\prime}}^{\bar{f}}$ is an isomorphism.

As a consequence, for every $x^{\prime} \in Z^{\prime}$, we have

a) $\theta_{g, x^{\prime}}^{\bar{f}}$ does not depend on $\bar{f}$;

b) $\theta_{g, x^{\prime}}^{\bar{f}}$ is an isomorphism.

The difficulty is in finding enough good immersions in $X$. This is where the Cohen-Macaulay property helps. In the flat topology on $X$ we have a plentiful supply of good immersions, and then faithful flat descent gives the rest. We bring the above ideas down to earth as follows. 


\section{P. SASTRY}

Let $x \in X$ be a point closed in its fiber over $Y$. Let

- $y=f(x), k=\mathcal{O}_{Y, y} / \mathfrak{m}_{y}$,

- $X_{k}=X \times_{Y} \operatorname{Spec} k, \bar{x} \in X_{k}$ the closed point corresponding to $x \in X$,

- $A=\widehat{\mathcal{O}}_{Y, y}, T=\operatorname{Spec} A$,

- $u: T \rightarrow Y$ the natural map.

The map $u: T \rightarrow Y$ induces the diagram in Remark 3.3.2 as well as a 'compactified' version of that diagram

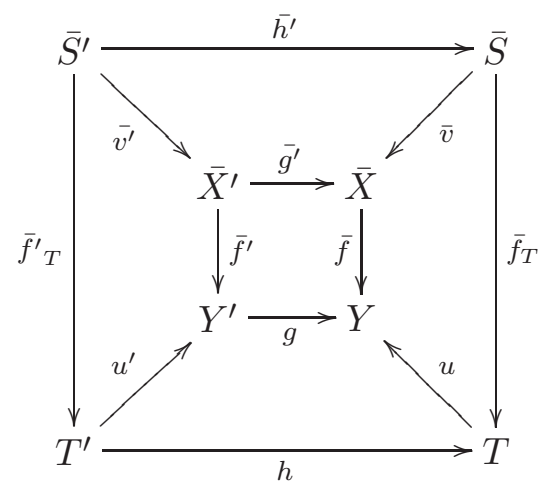

with $\bar{f}^{\prime}, \bar{f}_{T}^{\prime}, \bar{g}^{\prime}, \bar{h}^{\prime}, \bar{v}$ and $\bar{v}^{\prime}$ being the compactifications of $f^{\prime}, f_{T}^{\prime}, g^{\prime}, h^{\prime}, v$ and $v^{\prime}$ induced by the compactification $\bar{f}$ of $f$.

Let $s \in S$ be the point corresponding to $\bar{x} \in X_{k}$. Since $X_{k}$ is Cohen-Macaulay, we can find an $\mathcal{O}_{X_{k}, \bar{x}}$-sequence $\bar{t}_{1} \ldots, \bar{t}_{r} \in \mathfrak{m}_{\bar{x}}$. There is an affine open neighborhood $U=\operatorname{Spec} R$ of $s \in S$ such that the closed subscheme of $U \times_{T}$ Spec $k$ given by the vanishing of the $\bar{t}_{i}$ is supported only on $\bar{x}$. According to [Mat86, p. 177, Corollary to Theorem 22.5], we may choose $U$ to be such that we can lift $\bar{t}_{1}, \ldots, \bar{t}_{r}$ to an $R$-sequence $t_{1}, \ldots, t_{r}$. By shrinking the affine neighborhood $U$ of $s$ if necessary, we may also assume that, if $Z$ is the closed subscheme of $U$ defined by the $t$ 's, then $Z$ has only one connected component. We claim that the map $Z \hookrightarrow \bar{S}$ is also a closed immersion. This would follow if $Z$ is shown to be finite (and hence proper) over $Y$. Now $T$ is the spectrum of a complete local ring and the fiber of $Z \rightarrow T$ over the closed point of $T$ has only one point (by choice of $U$ ). Since $Z$ has only one connected component, it follows that $Z$ is finite over $Y$ (cf. [GD61a, (6.2.5) and (6.2.6)]). Clearly $Z \stackrel{\jmath}{\hookrightarrow} S$ is a good immersion for $f_{T}$. By Remark 3.3.2, especially (3.7), we have

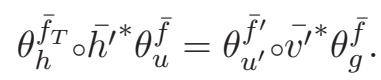

Now $g^{\prime-1}(x)=h^{\prime-1}(s)$. For $s^{\prime} \in h^{\prime-1}(s)$, let $x^{\prime}$ denote the corresponding point in $g^{\prime-1}(x)$. We then have that the completion of ${\overline{v^{\prime}}}^{*} \theta_{g}^{\bar{f}}$ at $s^{\prime} \in S^{\prime}$ is equal to the completion of $\theta_{g}^{\bar{f}}$ at $x^{\prime} \in X^{\prime}$. Since $u$ and $u^{\prime}$ are flat, therefore $\theta_{u}^{\bar{f}}$ and $\theta_{u^{\prime}}^{\bar{f}^{\prime}}$ are isomorphisms by the flat base change theorem for the twisted inverse image functor (cf. [Lip99, p. 120, Corollary (4.3)] and [Ver69, pp. 394-395, Theorem 2]). Moreover, by Remark 2.3.4, they are also independent of the compactification $\bar{f}$. Now, from arguments we gave earlier in this proof, for every $s^{\prime} \in h^{\prime-1}(s), \theta_{h, s^{\prime}}^{\bar{f}_{T}}$ is independent of $\bar{f}$ and is an isomorphism. Equation (3.8) together with these facts imply that $\theta_{g, x^{\prime}}^{\bar{f}}$ is independent of $\bar{f}$ and is an isomorphism for every $x^{\prime} \in g^{\prime-1}(x)$. Since $x \in X$ was an arbitrary point closed in its fiber, therefore (as $x$ varies) such $x^{\prime}$ form a collection that contains all points of $X^{\prime}$ which are closed in their fibers with respect to $f^{\prime}$. Parts a and b of Theorem 2.3.3 are immediate.

Remark 3.3.4. The Cohen-Macaulay hypothesis has been used in finding a good immersion $Z \hookrightarrow S$ over $T$. We will see later that the hypothesis is also used for getting the various local duality properties of $\operatorname{res}_{Z}, g^{\#} \operatorname{res}_{Z^{\prime}}$, etc. 


\section{Base Change For COHEN-MaCAulay MaPs}

\section{The fundamental local isomorphism; adjunction}

One of the technical devices we use in this paper is the compatibility of local and global duality. In greater detail, if $Z \rightarrow Y$ is a proper map which factors as a closed immersion $Z \hookrightarrow X$ followed by a proper map $X \rightarrow Y$, then we wish to understand duality for $Z$ (i.e., for $Z \rightarrow Y$ ) and (in essence) all its thickenings in $X$ in terms of duality for $X \rightarrow Y$. For this paper the most important case is that when $Z \hookrightarrow X$ is a regular immersion. Roughly speaking, $\S 4.1$ discusses duality for a regular immersion and $\S 4.2$ brings in the other piece, namely, the map $X \rightarrow Y$.

\subsection{The fundamental local isomorphism}

The fundamental local isomorphism (4.3) can be interpreted as giving an explicit description of $\jmath \mathcal{O}_{X}$ for a closed regular immersion $\jmath: Z \hookrightarrow X$ in terms of the top exterior power of the normal bundle of $Z$ in $X$. In this section we give a local description of (4.3) when $X$ is affine and $Z$ is given globally by the vanishing of a Koszul-regular sequence. Let $R$ be a ring and $I$ an ideal of $R$ generated by a Koszul-regular $R$-sequence $\boldsymbol{t}=\left(t_{1}, \ldots, t_{r}\right)$. Let $B=R / I$, and $N_{B / R}=\bigwedge_{B}^{r} \operatorname{Hom}_{B}\left(I / I^{2}, B\right)=$ $\operatorname{Hom}_{B}\left(\bigwedge_{B}^{r} I / I^{2}, B\right)$. For $t \in I$, let $\bar{t}$ denote its image in $I / I^{2}$. Now, $\bigwedge_{B}^{r} I / I^{2}$ is a free $B$-module of rank one with $\bar{t}_{1} \wedge \cdots \wedge \bar{t}_{r}$ a generator. Denote by

$$
\frac{1}{\left(t_{1}, \ldots, t_{r}\right)} \in N_{B / R}
$$

the dual generator (which sends $\bar{t}_{1} \wedge \cdots \wedge \bar{t}_{r}$ to $1 \in B$ ). Let $K^{\bullet}=K^{\bullet}(\boldsymbol{t}, R$ ) denote the Koszul cohomology complex on $\boldsymbol{t}$. There is (from comments in the previous subsection), a complex of free $R$-modules $C^{\bullet}$, concentrated in degrees $-r$ to 0 , such that

$$
K^{\bullet}=\operatorname{Hom}_{R}^{\bullet}\left(C^{\bullet}, R\right) .
$$

In view of the sign conventions for Hom $\bullet$, the complex $C^{\bullet}$ is not the Koszul homology complex (concentrated in degrees $-r$ to 0 ) on $\boldsymbol{t}$, though it is canonically isomorphic to it, and as such it resolves the $R$-module $B$. It is well known that $K^{\bullet}$ resolves $N_{B / R}[-r]$, the map $K^{r}=R \rightarrow$ $N_{B / R}$ being the one which sends 1 to $1 /\left(t_{1}, \ldots, t_{r}\right)$. In the category $D^{+}\left(\operatorname{Mod}_{R}\right)$ we thus have two isomorphisms,

$$
\begin{aligned}
& B \stackrel{\sim}{\longrightarrow} C^{\bullet}, \\
& N_{B / R}[-r] \stackrel{\sim}{\longrightarrow} K^{\bullet} .
\end{aligned}
$$

For $M^{\bullet} \in D^{+}\left(\operatorname{Mod}_{R}\right)$ we have functorial isomorphisms

$$
\begin{aligned}
& M^{\bullet} \stackrel{L}{\otimes} N_{B / R}[-r] \stackrel{\sim}{\longrightarrow} M^{\bullet} \otimes K^{\bullet} \\
& \stackrel{\sim}{\longrightarrow} \operatorname{Hom}_{R}^{\bullet}\left(C^{\bullet}, M^{\bullet}\right) \\
& \stackrel{\sim}{\longrightarrow} R \operatorname{Hom}_{R}^{\bullet}\left(B, M^{\bullet}\right) \text {. }
\end{aligned}
$$

The resulting isomorphism between the complexes $M^{\bullet} \otimes_{R}^{L} N_{B / R}[-r]$ and $R \operatorname{Hom}_{R}^{\bullet}\left(B, M^{\bullet}\right.$ ) (obtained by composing the above isomorphisms together) is well known to be independent of the Koszulregular $R$-sequence generating $I$ (even though the intermediate steps do depend on $\boldsymbol{t}$ ). The above is the local aspect of a more global isomorphism which we now describe. Let $Z \stackrel{\jmath}{\hookrightarrow} X$ be a (regular) closed immersion, i.e. the ideal $\mathcal{I}$ of $\mathcal{O}_{X}$ giving the immersion $\mathrm{\jmath}$ is locally generated by an $\mathcal{O}_{X^{-}}$ sequence. Let $\mathcal{N}_{\jmath}$ denote the top wedge product of the normal bundle of the immersion $\jmath: Z \hookrightarrow X$. Then, using [Har66, p. 74, Proposition I.7.4], Hartshorne proves in [Har66, p. 180, Corollary III.7.3] that in $D_{\mathrm{qc}}^{+}(X)$ we have a functorial isomorphism, the fundamental local isomorphism

$$
\mathcal{G}^{\bullet} \stackrel{L}{\otimes}_{\mathcal{O}_{X}} J_{*} \mathcal{N}_{\jmath}[-r] \stackrel{\sim}{\longrightarrow} R \mathcal{H o m}_{\mathcal{O}_{X}}\left(\jmath_{*} \mathcal{O}_{Z}, \mathcal{G}^{\bullet}\right)
$$




\section{P. SASTRY}

which, when restricted to the case considered above, gives (4.2). Our sign conventions are different from Hartshorne's but the proof given in [Har66] works in our situation also.

\subsection{Adjunction}

The principal result of this section is Proposition 4.2.1. To motivate it, consider the situation described in the beginning of $\S 4$ and let $h$ and $f$ be the maps $Z \rightarrow Y$ and $X \rightarrow Y$. Assume $Z \hookrightarrow X$ is a regular immersion. The fundamental local isomorphism (4.3) allows us to describe $h$ ! in terms of $f^{!}$and (the top exterior power of) the normal bundle to $Z$ in $X$. In broad terms, in view of the local nature of $f^{!}$and the nature of the normal bundle, this description of $h^{!}$does not depend on the nature of $X$ 'away from $Z$ ', but only on how it 'looks' near $Z$. However, it is not a priori clear that the resulting trace $h_{*} h^{!} \rightarrow 1_{D_{\mathrm{qc}}^{+}(Y)}$ depends only on the description of $X$ 'around $Z$ ', although it is reasonable to expect this. Roughly speaking, Proposition 4.2.1 justifies this expectation. In $\S 5$ we make this rough philosophy more precise by taking (essentially) all possible thickenings of $Z$ in $X$ to get local duality in the special case where $X \rightarrow Y$ is Cohen-Macaulay in a (Zariski) neighborhood of $Z$ and $Z \hookrightarrow X$ is a good immersion for $X \rightarrow Y$.

Let $\jmath: Z \hookrightarrow X$ be a closed immersion of schemes. We recall the explicit description of duality for the map J. Let $\mathcal{E}^{\bullet}$ be a bounded below complex of quasi-coherent, injective $\mathcal{O}_{X}$-modules, and $\mathcal{J}^{\bullet}$ the injective $\mathcal{O}_{Z}$-complex satisfying $\jmath_{*} \mathcal{J}^{\bullet}=\mathcal{H}_{o} m_{\mathcal{O}_{X}}\left(\jmath_{*} \mathcal{O}_{Z}, \mathcal{E}^{\bullet}\right)$. The adjoint properties of Hom and $\otimes$ give, for any bounded below complex $\mathcal{F}^{\bullet}$, a functorial isomorphism of $\mathcal{O}_{Z}$-modules, $\mathcal{H o m}_{\mathcal{O}_{Z}}\left(\mathcal{F}^{\bullet}, \mathcal{J}^{\bullet}\right) \stackrel{\sim}{\longrightarrow} \mathcal{H o m}_{\mathcal{O}_{X}}\left(\jmath_{*} \mathcal{F}^{\bullet}, \mathcal{E}^{\bullet}\right)$. Since $\jmath_{*} \mathcal{J}^{\bullet}$ is a complex of injective $\mathcal{O}_{X}$-modules, we have that $\jmath^{\prime} \mathcal{E}^{\bullet} \simeq \mathcal{J}^{\bullet}$, and under this identification, the trace map $\jmath_{*} J^{!} \mathcal{E}^{\bullet} \rightarrow \mathcal{E}^{\bullet}$ is the natural inclusion $\jmath_{*} \mathcal{J}^{\bullet}=\mathcal{H o m}_{\mathcal{O}_{X}}\left(\jmath_{*} \mathcal{O}_{Z}, \mathcal{E}^{\bullet}\right) \hookrightarrow \mathcal{E}^{\bullet}$.

Now suppose $\jmath: Z \hookrightarrow X$ is a regular immersion. For $\mathcal{G}^{\bullet} \in D_{\mathrm{qc}}^{+}(X)$, set $\jmath^{\dagger} \mathcal{G}^{\bullet}=L \jmath^{*} \mathcal{G}^{\bullet} \otimes^{L} \mathcal{O}_{Z} \mathcal{N}_{\jmath}[-r]$. In view of the fundamental local isomorphism (4.3) and our description of duality for $\jmath$ we have the adjunction isomorphism:

$$
j^{!} \stackrel{\sim}{\longrightarrow} j^{\dagger} .
$$

If $f: X \rightarrow Y$ is a finite type separated map such that $h=f \circ \jmath: Z \rightarrow Y$ is proper, then we have a map

$$
\tau_{Z}: R h_{*} J^{\dagger} \longrightarrow R_{Z} f_{*}
$$

arising from isomorphism (4.3) and the fact that $R \mathcal{H o m}_{\mathcal{O}_{X}}\left(\jmath_{*} \mathcal{O}_{Z}, \cdot\right)$ is a 'subfunctor' of $R \underline{\Gamma}_{Z}$ (and the fact that $\left.R h_{*}=R f_{* \circ} J_{*}\right)$. Note also that the isomorphism (4.4) gives us

$$
h^{!} \stackrel{\sim}{\longrightarrow} j^{\dagger} f^{!} .
$$

We would like to explicate the map $R h_{*} j^{\dagger} f^{!} \rightarrow 1_{D_{\mathrm{qc}}^{+}(Y)}$ arising from the trace map $T_{h}: R h_{*} h^{!} \rightarrow$ $1_{D_{\mathrm{qc}}^{+}(Y)}$ and (4.6). To that end, let $(\imath, \bar{f})$ be a compactification of the (not necessarily proper) map $f$. For $\mathcal{G}^{\bullet} \in D_{\mathrm{qc}}^{+}(Y)$ define

$$
T_{h}^{\prime}\left(\mathcal{G}^{\bullet}\right): R h_{*} J^{\dagger} f^{!} \mathcal{G}^{\bullet} \longrightarrow \mathcal{G}^{\bullet}
$$

by the composite

$$
R h_{*} \jmath^{\dagger} f^{!} \mathcal{G}^{\bullet} \stackrel{\tau_{Z}}{\longrightarrow} R_{Z} f_{*} f^{!} \mathcal{G}^{\bullet} \stackrel{\sim}{\longrightarrow} R_{Z} \bar{f}_{*} \bar{f}^{!} \mathcal{G}^{\bullet} \longrightarrow R \bar{f}_{*} \bar{f}^{\prime} \mathcal{G}^{\bullet} \stackrel{T_{\bar{f}}}{\longrightarrow} \mathcal{G}^{\bullet}
$$

We now come to the main point of this section.

Proposition 4.2.1.

a) $T_{h}^{\prime}$ does not depend on the compactification $(\imath, \bar{f})$. 
b) The composite

is the trace map $T_{h}$.

$$
R h_{*} h^{!} \stackrel{(4.6)}{\longrightarrow} R h_{*} j^{\dagger} f^{!} \stackrel{T_{h}^{\prime}}{\longrightarrow} 1_{D_{\mathrm{qc}}^{+}(Y)}
$$

Proof. Part a is proved in exactly the same way in which Proposition 3.1.1 is proved. Part b follows from part a and the fact that (4.6) is an isomorphism, or alternatively from the identity $T_{h}=$ $T_{\bar{f}} \circ R \bar{f}_{*}\left(T_{\imath \jmath}\right)$. The functors can be composed only because we have implicitly made the identification $h^{!}=(\imath \jmath) ! f^{!}$as in (1.10).

\section{Local duality}

\subsection{A perfect pairing}

For this subsection, we assume $f: X \rightarrow Y$ is a separated Cohen-Macaulay map of relative dimension $r$. We also assume that we are given a good immersion $\jmath: Z \hookrightarrow X$ for $f$. Set $h=f \circ \jmath: Z \rightarrow Y$.

Now by Lemma 3.1.5 $h: Z \rightarrow Y$ is flat. Since $h$ is finite, it then follows that $h$ is Cohen-Macaulay of relative dimension 0 . This means $H^{i}\left(h ! \mathcal{O}_{Y}\right)=0$ for $i \neq 0$. This gives a canonical isomorphism $h^{!} \mathcal{O}_{Y} \simeq Q\left(H^{0}\left(h^{!} \mathcal{O}_{Y}\right)\right)\left(Q=Q_{Z}: K_{\mathrm{qc}}^{+}(Z) \rightarrow D_{\mathrm{qc}}^{+}(Z)\right.$ is the 'localization' functor $)$. We claim, on the other hand, that there is a natural map

$$
L \jmath^{*} \omega_{f} \stackrel{L}{\otimes} \mathcal{O}_{Z} \mathcal{N}_{\jmath}[-r] \rightarrow Q\left(\jmath^{*} \omega_{f}[r] \otimes_{\mathcal{O}_{Z}} \mathcal{N}_{\jmath}[-r]\right)
$$

whose 0th cohomology is an isomorphism. This claim, in conjunction with (4.6) and the isomorphism $h^{!} \mathcal{O}_{Y} \simeq Q\left(H^{0}\left(h ! \mathcal{O}_{Y}\right)\right)$, allows us to make the identification

$$
h^{!} \mathcal{O}_{Y}=Q\left(\jmath^{*} \omega_{f}[r] \otimes \mathcal{O}_{Z} \mathcal{N}_{\jmath}[-r]\right) .
$$

In order to prove our claim, let $F^{\bullet} \rightarrow \omega_{f}$ be an $\mathcal{O}_{X}$-flat resolution of $\omega_{f}$ (we may temporarily have to leave the category of quasi-coherent modules). This induces a map $\jmath^{*} F^{\bullet}[r] \otimes \mathcal{N}_{\jmath}[-r] \rightarrow$ $\jmath^{*} \omega_{f}[r] \otimes \mathcal{N}_{\jmath}[-r]$. Since tensor product is right exact, the last map gives an isomorphism on applying $H^{0}$. Since $\mathcal{N}_{\jmath}$ is locally free, we can make the identification

$$
Q\left(\jmath^{*} F^{\bullet}[r] \otimes_{\mathcal{O}_{Z}} \mathcal{N}_{\jmath}[-r]\right)=L \jmath^{*} \omega_{f}[r] \stackrel{L}{\otimes_{\mathcal{O}_{Z}}} \mathcal{N}_{\jmath}[-r]
$$

giving the claim.

Now set

$$
\stackrel{\circ}{\omega}_{h}=\jmath^{*} \omega_{f} \otimes \mathcal{O}_{Z} \mathcal{N}_{\jmath}
$$

and define

$$
\int_{h}^{\circ}: h_{*} \stackrel{\circ}{\omega}_{h} \longrightarrow \mathcal{O}_{Y}
$$

to be the composite

$$
h_{*} \stackrel{\circ}{\omega} \stackrel{h_{*} \theta_{r,-r}^{-1}}{\longrightarrow} h_{*}\left(\omega_{f}[r] \otimes \mathcal{N}_{\jmath}[-r]\right) \stackrel{T_{h}^{\prime}}{\longrightarrow} \mathcal{O}_{Y} .
$$

Here $\theta_{r,-r}$ is 'multiplication by $(-1)^{r}$ ' (see the definition of the map $\theta_{i j}$ in $\S 1.2$ ). The map $\int_{h}^{\circ}$ is $a$ priori a map in $D_{\mathrm{qc}}^{+}(Y)$, but since the source and target are concentrated in degree $0, \int_{h}^{\circ}$ is a map in $Y_{\mathrm{qc}}$. In the definition of $\int_{h}^{\circ}$ we have implicitly used the equality $R h_{*}=h_{*}$ ( $h$ is an affine map). The integral has another description. Taking the 0 th cohomology of the map $\tau_{Z}\left(\mathcal{O}_{Y}\right) \circ h_{*} \theta_{r,-r}^{-1}$ we get a map

$$
r_{Z}: \stackrel{\circ}{*}_{h} \rightarrow R_{Z}^{r} f_{*} \omega_{f}
$$




\section{P. SASTRY}

(Cf. also (C.11).) Then clearly

$$
\int_{h}^{\circ}=\operatorname{res}_{Z} \circ r_{Z}
$$

Proposition 5.1.1. The pair $\left(\stackrel{\circ}{\omega}_{h}, \int_{h}^{\circ}\right)$ defined by $(5.2)$ and $(5.3)$ is a dualizing pair for the map $h: Z \rightarrow Y$.

Proof. This is a consequence of Proposition 4.2 .1 and the definitions of $\stackrel{\circ}{\omega}_{h}$ and $\int_{h}^{\circ}$.

The following is a version of local duality

Proposition 5.1.2. The pairing given by the composite

$$
h_{*} \jmath^{*} \omega_{f} \otimes_{\mathcal{O}_{Y}} h_{*} \mathcal{N}_{\jmath} \longrightarrow h_{*}\left(\jmath^{*} \omega_{f} \otimes_{Z} \mathcal{N}_{\jmath}\right)=h_{*} \stackrel{\circ}{\omega}_{h} \stackrel{\int_{h}^{\circ}}{\longrightarrow} \mathcal{O}_{Z}
$$

is a perfect pairing of the $\mathcal{O}_{Y}$-modules $h_{*} \jmath^{*} \omega_{f}$ and $h_{*} \mathcal{N}_{\jmath}$.

Proof. From the definition of a good immersion, we may assume without loss of generality that $Y=\operatorname{Spec} A, X=\operatorname{Spec} R, Z=\operatorname{Spec} B$ and $B=R / I$, where $I$ is generated by an $R$-sequence $t_{1}, \ldots, t_{r}$. Our intent (clearly!) is to work with rings and modules, and we use the following dictionary $\stackrel{\circ}{\omega}_{h} \longleftrightarrow \stackrel{\circ}{\omega}_{B / A}, \int_{h}^{\circ} \longleftrightarrow \int_{B / A}^{\circ}, \omega_{f} \longleftrightarrow \omega_{R / A}$ and $\mathcal{N}_{\jmath} \longleftrightarrow N_{B / R}=N$. We have to show that the following composite arrow

$$
\left(B \otimes_{R} \omega_{R / A}\right) \otimes_{A} N \longrightarrow \stackrel{\circ}{\omega}_{B / A}=\left(B \otimes_{R} \omega_{R / A}\right) \otimes_{B} N \stackrel{\int_{B / A}^{\circ}}{\longrightarrow} A
$$

gives a perfect pairing between the $A$-modules $B \otimes_{R} \omega_{R / A}$ and $N$.

Since $B$ is flat and finite over $A$ (i.e. $B$ is a projective $A$-module), the composite (with $e=$ 'evaluation at 1 ')

$$
\operatorname{Hom}_{A}(B, A) \otimes_{A} B \longrightarrow \operatorname{Hom}_{A}(B, A) \otimes_{B} B=\operatorname{Hom}_{A}(B, A) \stackrel{e}{\longrightarrow} A
$$

is a perfect pairing of the $A$-modules $\operatorname{Hom}_{A}(B, A)$ and $B$. We will relate this pairing to the pairing stated in the proposition to reach the desired conclusion. We have a $B$-isomorphism

$$
\begin{gathered}
\varphi: N \stackrel{\sim}{\longrightarrow} B, \\
1 /\left(t_{1}, \ldots, t_{r}\right) \mapsto 1 .
\end{gathered}
$$

By the adjoint properties of Hom and $\otimes$, we see that the $B$-module functor $\operatorname{Hom}_{A}(\cdot, A)$ is represented by the pair $\left(\operatorname{Hom}_{A}(B, A), e\right)$. But it is also represented by $\left(\stackrel{\circ}{\omega}_{B / A}, \int_{B / A}^{\circ}\right)$ (for the pair $\left(\stackrel{\circ}{\omega}_{h}, \int_{h}^{\circ}\right)$ is dualizing). We therefore have an isomorphism

$$
\tilde{\psi}: \stackrel{\circ}{\omega}_{B / A}=\left(B \otimes_{R} \omega_{R / A}\right) \otimes_{B} N \stackrel{\sim}{\longrightarrow} \operatorname{Hom}_{A}(B, A)
$$

such that $e \circ \tilde{\psi}=\int_{B / A}^{\circ}$. Let

$$
\psi: B \otimes_{R} \omega_{R / A} \stackrel{\sim}{\longrightarrow} \operatorname{Hom}_{A}(B, A)
$$

be the $B$-isomorphism induced by $\tilde{\psi}$ and $\varphi$. Clearly $\tilde{\psi}=\psi \otimes_{B} \varphi$. We have a commutative diagram as follows.

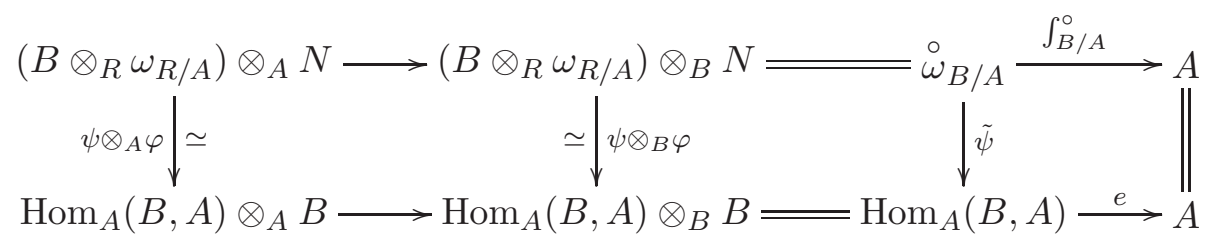

The bottom row is (5.6) which is a perfect pairing. The proposition follows. 


\subsection{Proof of Proposition 3.2.1}

Note that it is enough to prove Proposition 3.2.1 when $Y=\operatorname{Spec} A, X=\operatorname{Spec} R, Z=\operatorname{Spec} B, B=$ $R / I$, where $I$ is generated by a Koszul-regular $R$-sequence. This is from excision, the definition of a good immersion, and the fact that the residue map localizes well on the base (see Proposition 3.1.3, case b). We use the notations used in $\S 5.1$. The proof we give is the standard proof given, for example, in [Lip84, p. 68, Theorem (7.4)].

Let $\operatorname{res}_{I}: H_{I}^{r}\left(\omega_{R / A}\right) \rightarrow A$ be the $A$-map corresponding to the residue map res $Z$ (in other words $\operatorname{res}_{I}=\Gamma\left(Y, \operatorname{res}_{Z}\right)$ ). By Proposition 5.1.2 we have an isomorphism (of projective systems of $R$-modules)

$$
\omega_{R / A} / \boldsymbol{t}^{\alpha} \omega_{R / A} \stackrel{\sim}{\longrightarrow} \operatorname{Hom}_{A}\left(N_{\alpha}, A\right) .
$$

Taking projective limits, and using the isomorphism (C.12), we get

$$
\widehat{\omega_{R / A}} \stackrel{\sim}{\longrightarrow} \operatorname{Hom}_{A}\left(H_{I}^{r}(R), A\right) .
$$

By Remark C.2.2 (or Remark C.4.2) we have $H_{I}^{r}(R)=H_{\widehat{I}}^{r}(\widehat{R})$ and hence by the isomorphism (C.7) we have, for any finitely generated $\widehat{R}$-module $M$,

$$
H_{I}^{r}(R) \otimes_{\widehat{R}} M=H_{\widehat{I}}^{r}(M),
$$

whence a functorial isomorphism

$$
\operatorname{Hom}_{\widehat{R}}\left(M, \operatorname{Hom}_{A}\left(H_{I}^{r}(R), A\right)\right) \stackrel{\sim}{\longrightarrow} \operatorname{Hom}_{A}\left(H_{\widehat{I}}^{r}(M), A\right) .
$$

According to the isomorphism (5.7) (see also Remark C.4.2) this translates to a functorial isomorphism

$$
\operatorname{Hom}_{\widehat{R}}\left(M, \widehat{\omega}_{R / A}\right) \stackrel{\sim}{\longrightarrow} \operatorname{Hom}_{A}\left(H_{\widehat{I}}^{r}(M), A\right) .
$$

Now applying Proposition C.4.1, we see that if $M=\widehat{\omega}_{R / A}$ then the identity map on the left corresponds to $\operatorname{res}_{I}: H_{I}^{r}\left(\omega_{R / A}\right) \rightarrow A$, i.e. $\left(\widehat{\omega}_{R / A}, \operatorname{res}_{I}\right)$ represents the functor $\operatorname{Hom}_{A}\left(H_{\widehat{I}}^{r}(M), A\right)$ of finitely generated $\widehat{R}$-modules $M$. This completes the proof of Proposition 3.2.1.

\section{Base change for residues}

In this section we prove Proposition 3.2.2, thereby settling parts a and b of Theorem 2.3 .3 and hence also part a of Theorem 2.3.5. However, first we need some auxiliary constructions.

\subsection{Finite maps and base change}

Suppose $f: X \rightarrow Y$ is a finite Cohen-Macaulay map (or, what is the same thing, a finite flat map). Suppose further that we have a base change diagram.

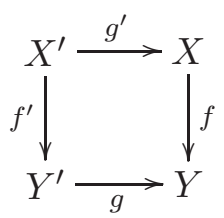

Lemma 6.1.1. The map $\theta_{g}^{f}: g^{\prime *} \omega_{f} \rightarrow \omega_{f^{\prime}}$ is an isomorphism.

Proof. By Remark 3.3.3, without loss of generality, we may assume that $Y=\operatorname{Spec} A, X=\operatorname{Spec} B$, $Y^{\prime}=\operatorname{Spec} A^{\prime}$ and $X^{\prime}=\operatorname{Spec} B^{\prime}\left(B^{\prime}=B \otimes_{A} A^{\prime}\right)$. In view of Remark 2.3.8, we may choose any convenient dualizing pairs for $f$ and $f^{\prime}$. We have very simple descriptions of $\left(\omega_{B / A}, \int_{B / A}\right)$ and $\left(\omega_{B^{\prime} / A^{\prime}}, \int_{B^{\prime} / A^{\prime}}\right)$ in this case (the notations are self-explanatory). Since Hom and $\otimes$ are adjoint 


\section{P. SASTRY}

functors,

$$
\begin{gathered}
\omega_{B / A}=\operatorname{Hom}_{A}(B, A), \\
\int_{B / A}: \omega_{B / A} \rightarrow A \quad(\varphi \mapsto \varphi(1)),
\end{gathered}
$$

have the necessary dualizing property for the map $f$. Similarly $\omega_{B^{\prime}} / A^{\prime}$ can be identified with $\operatorname{Hom}_{A^{\prime}}\left(B^{\prime}, A^{\prime}\right)$ and $\int_{B^{\prime} / A^{\prime}}$ with 'evaluation at $1^{\prime}$ '. The assertion that $\theta_{g}^{f}$ is an isomorphism reduces to checking that the natural map

$$
\omega_{B / A} \otimes_{A} A^{\prime}=\operatorname{Hom}_{A}(B, A) \otimes_{A} A^{\prime} \rightarrow \operatorname{Hom}_{A^{\prime}}\left(B \otimes_{A} A^{\prime}, A \otimes_{A} A^{\prime}\right)=\omega_{B^{\prime} / A^{\prime}}
$$

is an isomorphism. This reduction is made by noting that $\int_{B / A} \otimes_{A} A^{\prime}$ maps to $\int_{B^{\prime} / A^{\prime}}$ under this map and hence (6.1) is (the global section of) $\theta_{g}^{f}$. To check that the map is an isomorphism, we only have to note that $B$ is finite and flat over $A$, and hence it is a projective $A$-module.

Remark 6.1.2. It is worth pointing out that, in this case ( $f$ finite), $g^{*} f_{*}=f_{*}^{\prime} g^{\prime *}$, and hence we have an equality $g^{\#} \int_{f}=g^{*} \int_{f}$.

\subsection{Proof of Proposition 3.2.2}

By Remark 3.3.3 and the definition of good immersion, we may assume that $Y=\operatorname{Spec} A, Y^{\prime}=$ $\operatorname{Spec} A^{\prime}, X=\operatorname{Spec} R, X^{\prime}=\operatorname{Spec} R^{\prime}$ and $Z$ is defined by an ideal $I$ of $R$ generated by a Koszul-regular $R$-sequence $\boldsymbol{t}=\left(t_{1}, \ldots, t_{r}\right)$. Note that since $R$ and $B=R / I$ are flat over $A$, the extension of $\boldsymbol{t}$ to $R^{\prime}$ is a Koszul-regular $R^{\prime}$-sequence. Let $I^{\prime}=I R^{\prime}$ and $B^{\prime}=R^{\prime} / I^{\prime}$. Let $Z^{\prime}=\operatorname{Spec} B^{\prime}$. For a sequence of positive integers $\alpha=\left(\alpha_{1}, \ldots, \alpha_{r}\right)$, let $B_{\alpha}=R / \boldsymbol{t}^{\alpha} R$ and $B_{\alpha}^{\prime}=B_{\alpha} \otimes_{A} A^{\prime}$. Let $N_{\alpha}=N_{B_{\alpha} / R}$ and $N_{\alpha}^{\prime}=N_{B_{\alpha}^{\prime} / R^{\prime}}$. Note that $N_{\alpha}^{\prime}=N_{\alpha} \otimes_{A} A^{\prime}$.

For typographical convenience set $\bar{\omega}_{\alpha}=\omega_{R / A} \otimes_{R} B_{\alpha}$ and $\bar{\omega}_{\alpha}^{\prime}=\omega_{R^{\prime} / A^{\prime}} \otimes_{R^{\prime}} B_{\alpha}^{\prime}$. According to Lemma 6.1.1 and (5.1) we have an isomorphism (corresponding to $\theta_{g}^{h_{\alpha}}$ where $h_{\alpha}:$ Spec $B_{\alpha} \rightarrow Y$ is the structural morphism)

$$
\left(\bar{\omega}_{\alpha} \otimes_{B_{\alpha}} B_{\alpha}^{\prime}\right) \otimes_{B_{\alpha}^{\prime}} N_{\alpha}^{\prime}=\left(\bar{\omega}_{\alpha} \otimes_{B_{\alpha}} N_{\alpha}\right) \otimes_{B_{\alpha}} B_{\alpha}^{\prime} \stackrel{\sim}{\longrightarrow} \bar{\omega}_{\alpha}^{\prime} \otimes_{B_{\alpha}^{\prime}} N_{\alpha}^{\prime} .
$$

Since $N_{\alpha}^{\prime}$ is a free $B_{\alpha}^{\prime}$-module of rank $1,(6.2)$ induces an isomorphism

$$
\bar{\omega}_{\alpha} \otimes_{B_{\alpha}} B_{\alpha}^{\prime} \stackrel{\sim}{\longrightarrow} \bar{\omega}_{\alpha}^{\prime}
$$

such that

We have a commutative diagram

$$
(6.2)=(6.3) \otimes_{B_{\alpha}^{\prime}} N_{\alpha}^{\prime}
$$

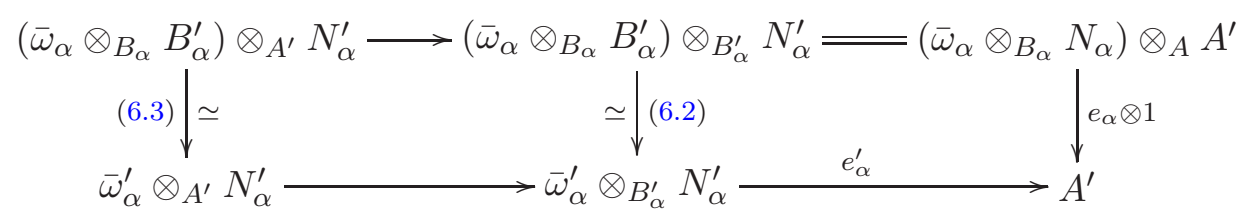

where $e_{\alpha}: \bar{\omega}_{\alpha} \otimes_{B_{\alpha}} N_{\alpha} \rightarrow A$ and $e_{\alpha}^{\prime}: \bar{\omega}_{\alpha}^{\prime} \otimes_{B_{\alpha}^{\prime}} N_{\alpha}^{\prime} \rightarrow A^{\prime}$ are the maps given by (5.3). By Proposition 5.1.2, the bottom row is a perfect pairing between the $A^{\prime}$-modules $\bar{\omega}_{\alpha}^{\prime}$ and $N_{\alpha}^{\prime}$. It follows that the composite

$$
\begin{aligned}
\left(\bar{\omega}_{\alpha} \otimes_{A} A^{\prime}\right) \otimes_{A^{\prime}} N_{\alpha}^{\prime} & \longrightarrow\left(\bar{\omega}_{\alpha} \otimes_{A} A^{\prime}\right) \otimes_{B_{\alpha}^{\prime}} N_{\alpha}^{\prime} \\
& =\left(\bar{\omega}_{\alpha} \otimes_{B_{\alpha}} N_{\alpha}\right) \otimes_{A} A^{\prime} \\
& \stackrel{e_{\alpha} \otimes 1}{\longrightarrow} A^{\prime}
\end{aligned}
$$

is a perfect pairing between the $A^{\prime}$-modules $\bar{\omega}_{\alpha} \otimes_{A} A^{\prime}$ and $N_{\alpha}^{\prime}$. 


\section{Base Change for Cohen-Macaulay maps}

To reduce notational clutter, let $\omega=\omega_{R / A}$ and $\omega^{\prime}=\omega_{R / A} \otimes_{A} A^{\prime}$ (not to be confused with $\omega_{R^{\prime} / A^{\prime}}$ ) and let $\widehat{(\cdot)}$ denote $I$-adic completion (which for $R^{\prime}$ modules is the same as $I^{\prime}=I R^{\prime}$-adic completion). If $M$ (respectively $N$ ) is a finitely generated $R$ (respectively $R^{\prime}$ ) module, then we will make the identifications $H_{I}^{r}(M)=H_{\widehat{I}}^{r}(\widehat{M})$ and $H_{I^{\prime}}^{r}(N)=H_{\bar{I}^{\prime}}^{r}(\widehat{N})$ without comment (cf. Remarks C.2.2 and C.4.2). As in the proof of Proposition 3.2.1 in $\S 5.2$, the perfect pairing induced by (6.5) gives, via (C.12), an isomorphism of $\widehat{R}^{\prime}$-modules

$$
\widehat{\omega}^{\prime} \stackrel{\sim}{\longrightarrow} \operatorname{Hom}_{A^{\prime}}\left(H_{I^{\prime}}^{r}\left(R^{\prime}\right), A^{\prime}\right)
$$

Moreover, if res* $: H_{I^{\prime}}^{r}\left(\omega^{\prime}\right) \rightarrow A^{\prime}$ is the composite $H_{I^{\prime}}^{r}\left(\omega^{\prime}\right) \simeq H_{I^{\prime}}^{r}\left(R^{\prime}\right) \otimes{\widehat{R^{\prime}}}_{\widehat{\omega}^{\prime}} \rightarrow A^{\prime}$ where the first isomorphism is (C.7) and the second map is the contraction induced by (6.6), then $\left(\widehat{\omega}^{\prime}\right.$, res $\left.{ }^{*}\right)$ represents the functor $\operatorname{Hom}_{A^{\prime}}\left(H_{\widehat{I}^{\prime}}^{r}(M), A^{\prime}\right)$ of finitely generated $\widehat{R}^{\prime}$-modules $M$. The proof of Proposition 3.2.2 will be complete once we show that the following diagram

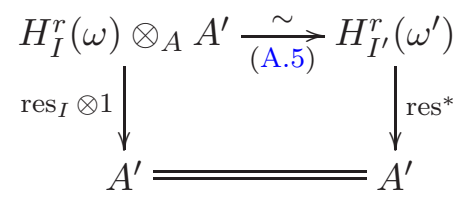

commutes. According to Proposition C.4.1, part b, we have a commutative diagram as follows.

$$
\begin{aligned}
& \underset{\alpha}{\lim }\left(\omega \otimes_{R} N_{\alpha}\right) \otimes_{A} A^{\prime}=\underset{\alpha}{\lim }\left(\omega^{\prime} \otimes_{R^{\prime}} N_{\alpha}^{\prime}\right) \\
& (\mathrm{C} .11) \downarrow \simeq \quad \simeq \downarrow(\mathrm{C} .11) \\
& H_{I}^{r}(\omega) \otimes_{A} A^{\prime} \underset{(\mathrm{A} .5)}{\sim} H_{I^{\prime}}^{r}\left(\omega^{\prime}\right)
\end{aligned}
$$

We check, from the definition of res*, that (6.8) implies the commutativity of (6.7). This concludes the proof of Proposition 3.2.2.

Remark 6.2.1. At this point we have proved completely parts a and b of Theorem 2.3.3 and hence also part a of Theorem 2.3.5 (see $\S 3.3$ ). Consequently, the assertion in Remark 3.3.2 remains true even if $f$ is not proper (or even separated), but under the added hypothesis that $f$ is Cohen-Macaulay. This is seen by locally compactifying $f$ in an $r$-proper (and possibly non-Cohen-Macaulay) way.

We prove two more results about the map $\theta_{g}^{f}$. The first result, that is to say Proposition 6.2.2, is a routine extension of Proposition 3.1.3 which we use a few times in this paper. The second result (Proposition 6.2.3) is a way of saying that the map (6.3) is $\Gamma\left(X^{\prime}, \theta_{g}^{f}\right) \otimes_{R^{\prime}} B_{\alpha}^{\prime}$. It is used in the proof of part c of Theorem 2.3.3.

For the rest of the section, let

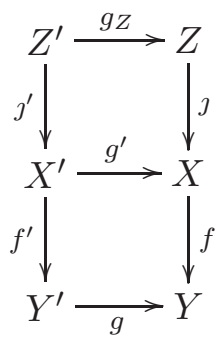

be a commutative diagram of cartesian squares with $f$ a separated Cohen-Macaulay map of relative dimension $r$ and $\jmath: Z \hookrightarrow X$ a closed immersion such that $Z$ is proper over $Y$.

Proposition 6.2.2. Suppose that one of the following conditions is satisfied:

a) the map $f$ is $r$-compactifiable; 


\section{P. SASTRY}

b) the map $g$ is flat;

c) the map $\mathrm{J}$ is a good immersion for $f$.

Then the diagram

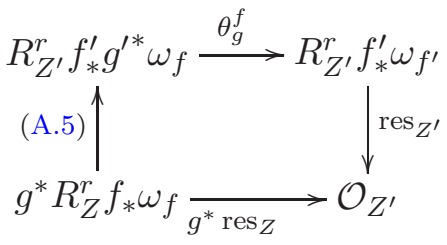

commutes.

Proof. Conditons a and b have already been dealt with in Proposition 3.1.3. Condition c will involve a number of base extensions of $Y$ and therefore, before moving on to condition c, we lay down some conventions and terminology which will help us keep track of the changing nature of the data $(f, g, Z)$. All triples $(p, q, W)$ which occur in the proof will be such that $p: P \rightarrow B$ is a separated Cohen-Macaulay map of relative dimension $r, q: B^{\prime} \rightarrow B$ is a map of schemes and $W$ is a closed subscheme of $P$ which is proper over $B$. We say that a triple $(p, q, W)$ has property $(\mathrm{R})$ if the conclusion of the proposition holds for $(p, q, W)$, i.e. if $q^{*} \operatorname{res}_{W} \circ(\mathrm{A} .5)=\operatorname{res}_{W^{\prime} \circ} \circ R_{W^{\prime}}^{r} p^{\prime}{ }_{*}\left(\theta_{q}^{p}\right)$ where $W^{\prime}=W \times_{B} B^{\prime}$ and $p^{\prime}$ is the projection $P \times_{B} B^{\prime} \rightarrow B^{\prime}$. Note that if $(p \mid U, q, W)$ has property (R) in some open neighborhood $U$ of $W$ in $P$ then so does $(p, q, W)$. We say two triples $(p, q, W)$ and $\left(p^{\prime}, \tilde{q}, W^{\prime}\right)$ are composable if $\tilde{q}$ has $B^{\prime}$ as its target (say $\left.\tilde{q}: B^{\prime \prime} \rightarrow B^{\prime}\right), p^{\prime}$ is the projection $P \times{ }_{B} B^{\prime} \rightarrow B^{\prime}$ and $W^{\prime}=W \times_{B} B^{\prime}$. In such a case the composite is defined as $(p, q, W) \circ\left(p^{\prime}, \tilde{q}, W^{\prime}\right)=(p, q \tilde{q}, W)$.

Now suppose that $W \hookrightarrow P$ is a good immersion and suppose further that the composite $(p, q, W) \circ\left(p^{\prime}, \tilde{q}, W^{\prime}\right)$ is defined. Let $p^{\prime \prime}, q^{\prime}$ and $\tilde{q}^{\prime}$ be respectively the projections $P \times_{B} B^{\prime \prime} \rightarrow B^{\prime \prime}$, $P \times_{B} B^{\prime} \rightarrow P, P \times_{B} B^{\prime \prime}=\left(P \times_{B} B^{\prime}\right) \times_{B^{\prime}} B^{\prime \prime} \rightarrow P \times_{B} B^{\prime}$ and let $W^{\prime \prime}=W \times_{B} B^{\prime \prime}$. Let $\rho_{q}: q^{*} R^{r} p_{*} \rightarrow R^{r} p_{*}^{\prime} q^{\prime *}, \rho_{\tilde{q}}: \tilde{q}^{*} R^{r} p_{*}^{\prime} \rightarrow R^{r} p^{\prime \prime}{ }_{*} \tilde{q}^{\prime *}$ and $\rho_{q \tilde{q}}:(q \tilde{q})^{*} R^{r} p_{*} \rightarrow R^{r} p^{\prime \prime}{ }_{*}\left(q^{\prime} \tilde{q}^{\prime}\right)^{*}$ be the maps arising from (A.5). We check using the generalized fraction description of (A.5) in Proposition C.3.1 that

$$
\rho_{q \tilde{q}}=\rho_{\tilde{q}} \circ \tilde{q}^{*} \rho_{q} .
$$

Moreover from the functoriality of $\rho_{\tilde{q}}$ we get the relation

$$
R^{r} p^{\prime \prime}{ }_{*}\left({\tilde{q^{\prime}}}^{*} \theta_{p}^{q}\right) \circ \rho_{\tilde{q}}=\rho_{\tilde{q}^{\circ}} \tilde{q}^{*} R^{r} p_{*}^{\prime}\left(\theta_{q}^{p}\right) .
$$

Using (6.10) and (6.11) and the relation $\theta_{\tilde{q}}^{p^{\prime}} \circ{\tilde{q^{\prime}}}^{*} \theta_{q}^{p}=\theta_{q \tilde{q}}^{p}$ (see (3.5)) we obtain the following two facts.

A) If $(p, q, W)$ and $\left(p^{\prime}, \tilde{q}, W^{\prime}\right)$ have property $(\mathrm{R})$ then so does the composite $(p, q, W) \circ\left(p^{\prime} \tilde{q}, W^{\prime}\right)$.

B) If $\left(p^{\prime}, \tilde{q}, W^{\prime}\right)$ and $(p, q, W) \circ\left(p^{\prime}, \tilde{q}, W^{\prime}\right)$ have property $(\mathrm{R})$ then

$$
\tilde{q}^{*}\left(q^{*} \operatorname{res}_{W} \circ \rho_{q}\right)=\tilde{q}^{*}\left(\operatorname{res}_{W^{\prime}} \circ R^{r} p_{*}^{\prime}\left(\theta_{q}^{p}\right)\right) .
$$

In other words $(p, q, W)$ has $(\mathrm{R})$ after applying $\tilde{q}^{*}$.

Now suppose condition c is met by $(f, g, Z)$. Then $g^{*} R_{Z}^{r} f_{*} \rightarrow R_{Z^{\prime}}^{r} f_{*}^{\prime} g^{\prime *}$ is an isomorphism (Proposition B.1.3) and hence by the definition of $g^{\#} \operatorname{res}_{Z}$ (see (3.4)), proving the proposition is equivalent to showing

$$
g^{\#} \operatorname{res}_{Z}=\operatorname{res}_{Z^{\prime}} \circ R_{Z^{\prime}}^{r} f_{*}^{\prime}\left(\theta_{g}^{f}\right) .
$$

Suppose first that $Y$ is the spectrum of a complete local ring $A$. Then by [GD61a, (6.2.5) and (6.2.6)], $Z$ is the disjoint union of closed subschemes, $Z=\coprod_{k=1}^{n} Z_{k}$, such that each $Z_{k}$ has a unique closed point $z_{k}$. Setting $Z_{k}^{\prime}=Z_{k} \times_{Y} Y^{\prime}$ we have $R_{Z}^{r} f_{*}=\oplus_{k=1}^{n} R_{Z_{k}}^{r} f_{*}, R_{Z^{\prime}}^{r} f_{*}^{\prime}=\oplus_{k=1}^{n} R_{Z_{k}^{\prime}}^{r} f_{*}^{\prime}$, $\operatorname{res}_{Z}=\oplus_{k=1}^{n} \operatorname{res}_{Z_{k}}$ and $\operatorname{res}_{Z^{\prime}}=\oplus_{k=1}^{n} \operatorname{res}_{Z_{k}^{\prime}}$. It is therefore enough to show that each $\left(f, g, Z_{k}\right)$ has property $(\mathrm{R})$. In other words it is enough to assume that $Z$ has exactly one closed point $z$. We can 


\section{BASE CHANGE FOR COHEN-MACAUlay MAPS}

always find an $r$-compactifiable open neighborhood of $z$ in $X$, and this neighborhood necessarily contains $Z$. By condition a $(f \mid U, g, Z)$ has $(\mathrm{R})$. It follows that $(f, g, Z)$ has $(\mathrm{R})$ when $Y$ is the spectrum of a complete local ring.

Since $\coprod_{y \in Y} Y^{\prime} \times_{Y} \operatorname{Spec} \widehat{\mathcal{O}}_{Y, y} \rightarrow Y^{\prime}$ is faithfully flat (the first scheme may not be Noetherian), in order to prove (6.12) it is enough to show that for an arbitrary $y \in Y$

$$
u^{\prime *}\left(g^{\#} \operatorname{res}_{Z}\right)=u^{\prime *}\left(\operatorname{res}_{Z^{\prime}} \circ R_{Z^{\prime}}^{r} f_{*}^{\prime}\left(\theta_{g}^{f}\right)\right),
$$

where $u^{\prime}$ is the map $Y^{\prime} \times_{Y} \operatorname{Spec} \widehat{\mathcal{O}}_{Y, y} \rightarrow Y^{\prime}$ (the notation $u^{\prime}$ is justified shortly). To that end let $T=\operatorname{Spec} \widehat{\mathcal{O}}_{Y, y}$ and let $u: T \rightarrow Y$ be the natural flat base extension and consider the resulting base change diagram (3.6). Note that the map $u^{\prime}$ in (3.6) agrees with our present usage. Let $Z_{T} \hookrightarrow S$ and $Z_{T}^{\prime} \hookrightarrow S^{\prime}$ be the good immersions induced by $Z \hookrightarrow X$. Since $u$ is flat therefore by condition $\mathrm{b}$ the triple $(f, u, Z)$ has property $(\mathrm{R})$, and since $T$ is the spectrum of a complete local ring the triple $\left(f_{T}, h, Z_{T}\right)$ also has $(\mathrm{R})$. It follows from fact $\mathrm{A}$ that the composite $(f, u, Z) \circ\left(f_{T}, h, Z_{T}\right)$ has property $(\mathrm{R})$. Now $(f, g, Z) \circ\left(f^{\prime}, u^{\prime}, Z^{\prime}\right)=(f, u, Z) \circ\left(f_{T}, h, Z_{T}\right)$ and $\left(f^{\prime}, u^{\prime}, Z^{\prime}\right)$ has $(\mathrm{R})$ (since $u^{\prime}$ is flat). We now appeal to fact $B$ to conclude that (6.13) is true.

If $\jmath$ is a good immersion, then arguing as we did for (6.2), we get an isomorphism

$$
\jmath^{\prime *} g^{\prime *} \omega_{f} \otimes_{\mathcal{O}_{Z^{\prime}}} \mathcal{N}_{\jmath^{\prime}}=g_{Z}^{*}\left(\jmath^{*} \omega_{f} \otimes_{\mathcal{O}_{Z}} \mathcal{N}_{\jmath}\right) \stackrel{\sim}{\longrightarrow} \jmath^{\prime *} \omega_{f^{\prime}} \otimes_{\mathcal{O}_{Z^{\prime}}} \mathcal{N}_{\jmath^{\prime}}
$$

arising from (5.1) and Lemma 6.1.1. Since $\mathcal{N}_{J^{\prime}}$ is an invertible $\mathcal{O}_{Z^{\prime}}$-module, this induces an isomorphism

$$
j^{\prime *} g^{\prime *} \omega_{f} \stackrel{\sim}{\longrightarrow} j^{\prime *} \omega_{f^{\prime}}
$$

It is reasonable to expect this isomorphism to be $\jmath^{\prime *} \theta_{g}^{f}$. This is indeed so. We state this assertion in the following equivalent form, which is convenient for applications in $\S 7$.

Proposition 6.2.3. Let $h$ be the composite $f \circ \jmath$ and $h^{\prime}$ the composite $f^{\prime} \circ \jmath^{\prime}$. Suppose $\jmath$ is a good immersion for $f$. Then the following diagram commutes

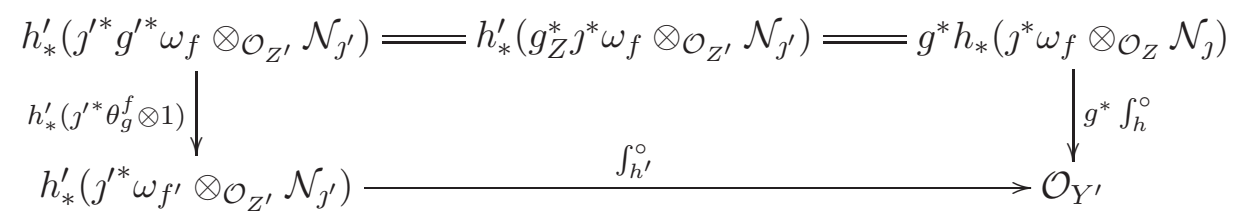

where $\int_{h}^{\circ}$ and $\int_{h^{\prime}}^{\circ}$ are as in (5.3).

Proof. Consider the following diagram.

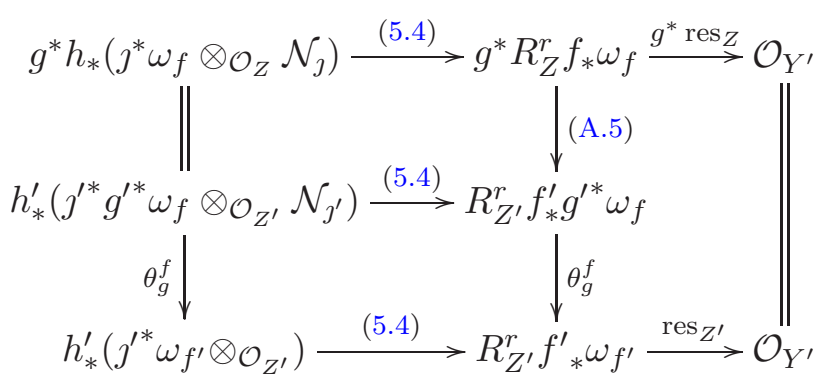

The top left rectangle commutes by Proposition C.4.1, part b. The bottom left rectangle clearly commutes. The rectangle on the right commutes by Proposition 6.2.2. According to (5.5), the top row composes to $g^{*} \int_{h}^{\circ}$ and the bottom row composes to $\int_{h^{\prime}}^{\circ}$. This completes the proof.

Remark 6.2.4. We expect Proposition 6.2.2 to be true without the conditions a, b or c (provided the basic conditions that $f$ is separated Cohen-Macaulay and $Z$ is proper over $Y$ are satisfied), but we do not know a proof. 


\section{P. SASTRY}

\section{The smooth case}

We now prove part c of Theorem 2.3.3. This will complete the proof of Theorems 2.3.3 and 2.3.5. We first give a quick review of the proof of the Verdier isomorphism (2.1).

\subsection{Verdier again}

Suppose $f: X \rightarrow Y$ is separated and Cohen-Macaulay of relative dimension $r$, and $\jmath: Z \hookrightarrow X$ is a good immersion for $f$, such that $h=f \circ \jmath$ is an isomorphism. (This implies that $f$ is smooth in a neighborhood of $Z$.) If $\varphi$ is the natural map $\mathcal{O}_{Y} \stackrel{\sim}{\longrightarrow} h_{*} \mathcal{O}_{Z}$, then $\varphi$ is an isomorphism and clearly $\left(\mathcal{O}_{Z}, \varphi^{-1}\right)$ is a dualizing pair for $h$. This means that the map

$$
\int_{h}^{\circ}: h_{*}\left(\jmath^{*} \omega_{f} \otimes \mathcal{O}_{Z} \mathcal{N}_{\jmath}\right) \rightarrow \mathcal{O}_{Y}
$$

is an isomorphism, and this induces (via $h_{*}^{-1}$ ) an isomorphism

$$
\jmath^{*} \omega_{f} \otimes_{\mathcal{O}_{Z}} \mathcal{N}_{\jmath} \stackrel{\sim}{\longrightarrow} \mathcal{O}_{Z} .
$$

Now suppose $f$ is separated and smooth, and $P=X \times_{Y} X$ with $p_{1}$ and $p_{2}$ the two projection maps $P \rightarrow X$ and $\delta: X \hookrightarrow P$ the diagonal map. Then $\delta$ is a good immersion for $p_{1}$ and (7.1) immediately gives us an isomorphism

$$
u_{f}: \delta^{*} \omega_{p_{1}} \otimes \mathcal{O}_{X} \mathcal{N}_{\delta} \stackrel{\sim}{\longrightarrow} \mathcal{O}_{X}
$$

We have already shown that $\theta_{f}^{f}: p_{2}^{*} \omega_{f} \rightarrow \omega_{p_{1}}$ is an isomorphism. Plugging this into the isomorphism $u_{f}$ above, and using the fact that $\delta^{*} p_{2}^{*}$ is the identity map on $X_{\mathrm{qc}}$, we get an isomorphism

$$
v_{f}: \omega_{f} \otimes \mathcal{O}_{X} \mathcal{N}_{\delta} \stackrel{\sim}{\longrightarrow} \mathcal{O}_{X} .
$$

Using the fact that $\mathcal{N}_{\delta}^{-1}=\Omega_{f}^{r}$, we get an isomorphism

$$
\omega_{f} \stackrel{\sim}{\longrightarrow} \Omega_{f}^{r}
$$

such that $v_{f}$ corresponds to the standard contraction $\Omega_{f}^{r} \otimes_{\mathcal{O}_{X}} \mathcal{N}_{\delta} \longrightarrow \mathcal{O}_{X}$. The isomorphism (7.2) is, up to a universal sign depending only on $r$, Verdier's isomorphism. In Verdier's proof [Ver69, p. 397, Theorem 3] the steps involved are essentially identical to the steps we took, and Verdier obtains an isomorphism

$$
\mathcal{O}_{X} \stackrel{\sim}{\longrightarrow} f^{!} \mathcal{O}_{Y} \otimes_{\mathcal{O}_{X}} \mathcal{N}_{\delta}[-r] .
$$

From here to concluding that $f^{!} \mathcal{O}_{Y}$ is isomorphic to $\Omega_{f}^{r}[r]$ is easy, but the exact isomorphism is not clear (to the author, at any rate). Part of the problem is the choice of the isomorphisms

$$
\begin{aligned}
& \mathcal{N}_{\delta}[-r] \otimes_{\mathcal{O}_{X}} \Omega_{f}^{r}[r] \stackrel{\sim}{\longrightarrow} \mathcal{O}_{X}, \\
& \Omega_{f}^{r}[r] \otimes_{\mathcal{O}_{X}} \mathcal{N}_{\delta}[-r] \stackrel{\sim}{\longrightarrow} \mathcal{O}_{X} .
\end{aligned}
$$

Suppose we fix these via the conventions in $\S 1.2$ (i.e. via $\theta_{r,-r}$ ) and by using the standard contraction $\Omega_{f}^{r} \otimes \mathcal{N}_{\delta} \rightarrow \mathcal{O}_{X}$. Even then we have choices. Do we tensor both sides of (7.3) on the right by $\Omega_{f}^{r}[r]$, and then use the contraction $\mathcal{N}_{\delta}[-r] \otimes \Omega_{f}^{r}[r] \rightarrow \mathcal{O}_{X}$ above, or do we pick the isomorphism such that (7.3) is compatible with (the inverse of) the above contraction $\Omega_{f}^{r}[r] \otimes \mathcal{N}_{\delta}[-r] \rightarrow \mathcal{O}_{X}$ ? These, incidentally, produce different answers. The choices made do not affect the result of part $\mathrm{c}$ of Theorem 2.3.3. We made our choice (7.2) with an eye on work we hope to return to in another paper on residues and traces. 


\subsection{Base change for smooth maps}

Consider the situation in part c of Theorem 2.3.3. Let $P=X \times_{Y} X$ and $P^{\prime}=X^{\prime} \times_{Y^{\prime}} X^{\prime}$. We then have a commutative diagram

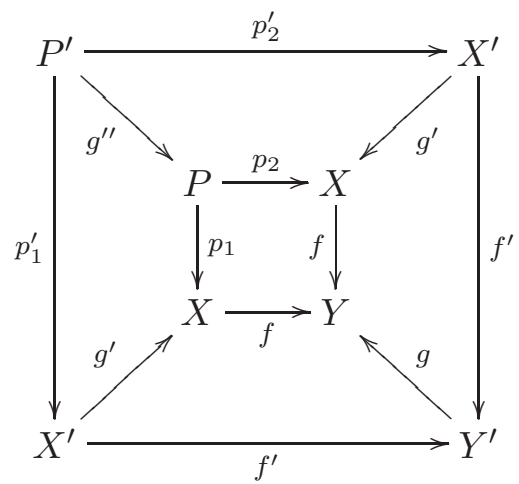

in which the outer square, the inner square, and the four trapeziums squeezed between them are all cartesian. According to Remark 6.2.1, the conclusions of Remark 3.3.2 apply in this case. Therefore the diagram

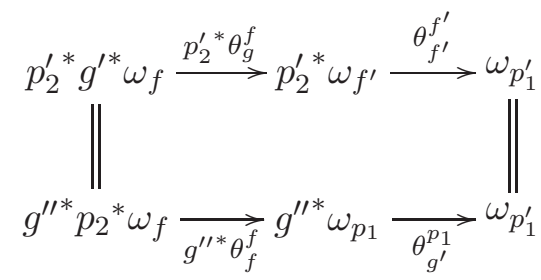

commutes. Let $\delta: X \rightarrow P$ and $\delta^{\prime}: X^{\prime} \rightarrow P^{\prime}$ be the respective diagonal maps. Note that we have an amalgamation of cartesian diagrams as follows.

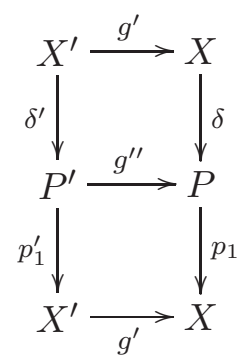

Let $\mathcal{N}=\mathcal{N}_{\delta}$ and $\mathcal{N}^{\prime}=\mathcal{N}_{\delta}^{\prime}$. Proposition 6.2.3 gives us a commutative diagram

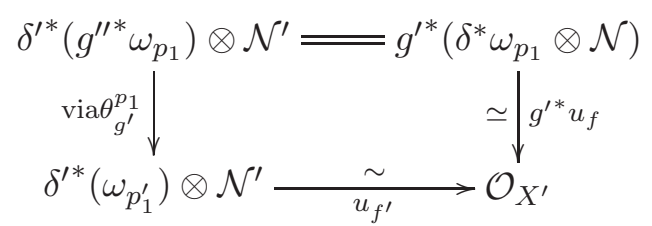

where $u_{f}$ and $u_{f^{\prime}}$ are the maps in $\S$ 7.1. Applying $\delta^{\prime *}$ to diagram (7.4) and using the equality $\delta^{\prime *} g^{\prime \prime *}=g^{\prime *} \delta^{*}$, we get a commutative diagram as follows.

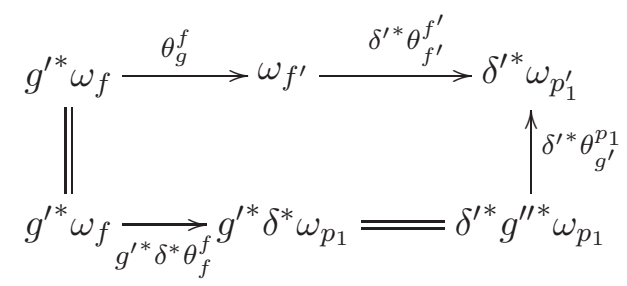




\section{P. SASTRY}

Consider the following diagram

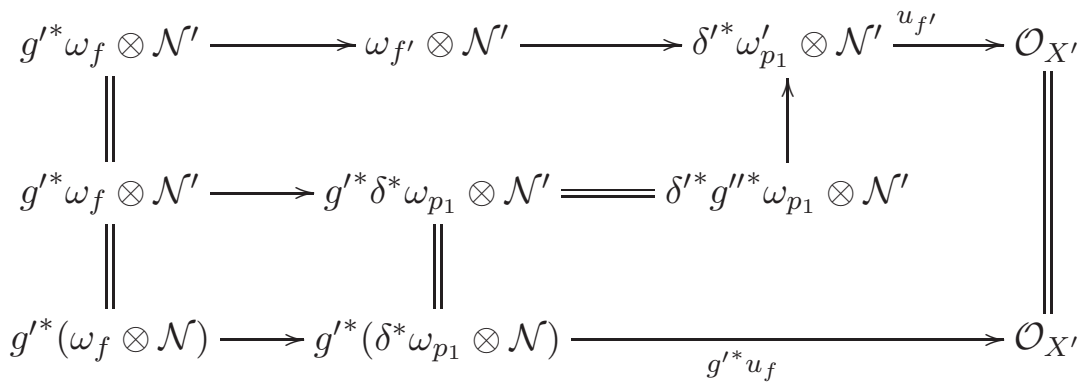

The top left rectangle commutes because of (7.6). The bottom left rectangle clearly commutes. The remaining subdiagram commutes because of (7.5). It follows that (7.7) commutes. This gives the commutativity of

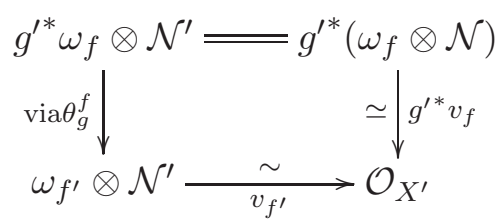

where $v_{f}$ and $v_{f^{\prime}}$ are as in $\S$ 7.1. Part c of Theorem 2.3.3 is immediate once we note that the identifications $g^{\prime *} \mathcal{N}=\mathcal{N}^{\prime}$ and $g^{\prime *} \Omega_{f}^{r}=\Omega_{f^{\prime}}^{r}$ are compatible.

\section{Explicit version of $\theta_{g}^{f}$}

This section is concerned with Theorem 2.3.6. In $\S 8.1$ the set-up is explained and the main ingredients (i.e. $\varphi, \varphi^{\prime}$ and $\psi_{g}^{f}$ ) required in the statement of the theorem are defined. The remainder of the section is taken up in proving the theorem.

\subsection{Dualizing sheaves and Ext sheaves}

Suppose $X \stackrel{\imath}{\hookrightarrow} P$ is a closed immersion of schemes. Let $\bar{\imath}$ be the morphism of ringed spaces $\left(X, \mathcal{O}_{X}\right) \rightarrow$ $\left(P, \imath_{*} \mathcal{O}_{X}\right)$, and $\operatorname{Mod}\left(\imath_{*} \mathcal{O}_{X}\right)$ the category of $\imath_{*} \mathcal{O}_{X}$-modules. Then we have functors $\bar{\imath}_{*}: \operatorname{Mod}\left(\mathcal{O}_{X}\right) \rightarrow$ $\operatorname{Mod}\left(\imath_{*} \mathcal{O}_{X}\right)$ and $\bar{\imath}^{*}: \operatorname{Mod}\left(\imath_{*} \mathcal{O}_{X}\right) \rightarrow \operatorname{Mod}\left(\mathcal{O}_{X}\right)$ which are adjoint, and $\bar{\imath}^{*}$ is exact (see [Har66, pp. 164-165]). Recall, and it is an elementary exercise in $R$ Hom, $\stackrel{L}{\otimes}$ adjointness to show this, that we may make the following identification:

$$
\imath^{!}=\imath^{*} R \mathcal{H o m}_{\mathcal{O}_{P}}\left(\imath_{*} \mathcal{O}_{X}, \cdot\right) .
$$

Note that $\bar{\imath}$ is flat and hence $\bar{\imath}^{*}=L \bar{\imath}^{*}$. With the identification (8.1), the trace map for $\imath$ (see (1.5)) can be identified with

$$
T_{\imath}: \bar{\imath}_{*} \imath^{!}=R \mathcal{H} \operatorname{Hom}_{\mathcal{O}_{P}}\left(\imath_{*} \mathcal{O}_{X}, \cdot\right) \stackrel{e}{\longrightarrow} R \mathcal{H o m}_{\mathcal{O}_{P}}\left(\mathcal{O}_{P}, \cdot\right)=1_{D_{\mathrm{qc}}^{+}(P)},
$$

where the arrow $e$ is 'evaluation at 1', or, what is the same thing, the map induced by the natural projection $\mathcal{O}_{P} \rightarrow \imath_{*} \mathcal{O}_{X}$ on the first argument of the bifunctor $R \mathcal{H o m} \bullet$.

Suppose

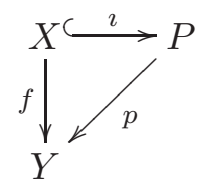

is a commutative diagram with $\imath$ a closed immersion, $f$ a Cohen-Macaulay map of relative dimension 


\section{BASE CHANGE FOR COHEN-MACAUlay MAPS}

$r$ and $p$ a smooth map of relative dimension $n=r+d$. Write

$$
\tilde{\omega}_{p}=\Omega_{p}^{n}
$$

and

$$
\tilde{\omega}_{f}\left(=\tilde{\omega}_{f}^{\Delta}\right)=\bar{\imath}^{*} \mathcal{E} x t_{\mathcal{O}_{P}}^{d}\left(\imath_{*} \mathcal{O}_{X}, \tilde{\omega}_{p}\right) .
$$

Suppose, for the next couple of lines, that $p$ is separated. Since $f^{!}=\imath^{!} p^{!}$(see (1.10)), we have an isomorphism $\omega_{f}[r] \stackrel{\sim}{\longrightarrow} \imath ! \tilde{\omega}_{p}[n]$. This isomorphism is compatible with open immersions into $X$, and hence we may drop the assumption that $p$ is separated to obtain this isomorphism, which we rewrite as

$$
\omega_{f}[r] \stackrel{\sim}{\longrightarrow} \bar{\imath}^{*} R \mathcal{H o m}_{\mathcal{O}_{P}}\left(\imath_{*} \mathcal{O}_{X}, \tilde{\omega}_{p}[n]\right)
$$

It follows that $\imath ! \tilde{\omega}_{p}[n]$ has cohomology only in the $-r$ th degree and hence, in $D_{\mathrm{qc}}^{+}(X)$, we have an isomorphism

$$
\begin{aligned}
\tilde{\varphi}: \tilde{\omega}_{f}[r] & =H^{-r}\left(\bar{\imath}^{*} R \mathcal{H o m}_{\mathcal{O}_{P}}\left(\imath_{*} \mathcal{O}_{X}, \tilde{\omega}_{p}[n]\right)\right)[r] \\
& =H^{-r}\left(\imath^{!} \tilde{\omega}_{p}[n]\right)[r] \\
& \stackrel{\sim}{\longrightarrow} ! \tilde{\omega}_{p}[n] .
\end{aligned}
$$

such that $H^{-r}(\tilde{\varphi})$ is the identity map on $\tilde{\omega}_{f}$ (see (8.1) for the second identification). This gives a natural isomorphism

$$
\varphi: \omega_{f} \stackrel{\sim}{\longrightarrow} \tilde{\omega}_{f},
$$

such that the diagram

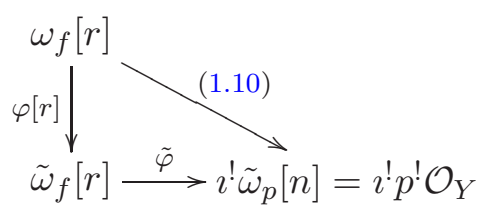

commutes whenever $p$ is separated (so that (1.10) is defined). Next, suppose $g: Y^{\prime} \rightarrow Y$ is a map of schemes and

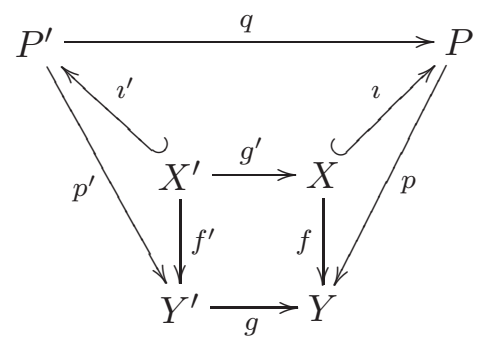

is the resulting base change diagram. Set

$$
\tilde{\omega}_{p^{\prime}}=\Omega_{f^{\prime}}^{n}
$$

and

$$
\tilde{\omega}_{f^{\prime}}={\overline{\iota^{\prime}}}^{*} \mathcal{E} x t_{\mathcal{O}_{P^{\prime}}}^{d}\left(\imath_{*}^{\prime} \mathcal{O}_{X^{\prime}}, \tilde{\omega}_{p^{\prime}}\right) .
$$

As before, there are natural isomorphisms

$$
\tilde{\varphi}^{\prime}: \tilde{\omega}_{f^{\prime}}[r] \longrightarrow \imath^{\prime} \tilde{\omega}_{p^{\prime}}[n]
$$

and

$$
\varphi^{\prime}: \omega_{f^{\prime}} \stackrel{\sim}{\longrightarrow} \tilde{\omega}_{f^{\prime}}
$$




\section{P. SASTRY}

such that

$$
\tilde{\varphi}^{\prime} \circ \varphi^{\prime}[r]=(1.10)
$$

when $p$ is separated.

We will describe now an explicit isomorphism

$$
\psi_{g}^{f}: g^{\prime *} \tilde{\omega}_{f} \stackrel{\sim}{\longrightarrow} \tilde{\omega}_{f^{\prime}},
$$

which, after identifying $\omega_{f}$ and $\omega_{f^{\prime}}$ with $\tilde{\omega}_{f}$ and $\tilde{\omega}_{f^{\prime}}$ via $\varphi$ and $\varphi^{\prime}$ respectively, we will show is $\theta_{g}^{f}$. (We remind the reader that this is the essential content of Theorem 2.3.6.) The map $\psi_{g}^{f}$ is best described locally. It will be clear that the various local isomorphisms glue. Therefore, assume (temporarily) that $p: P \rightarrow Y$ is quasi-projective. If $x \in X$ is a point closed in its fiber $f^{-1} f(x)$, then it is well known that the projective dimension of $\mathcal{O}_{X, x}$ over $\mathcal{O}_{P, \imath}(x)$ is $d$; see, for example, the proof of Lemma 1 in [Lip79, pp. 39-40] where this is worked out for $r=2$ (no changes are required for the general case). Since $X$ is quasi-projective, one has a resolution of $\imath_{*} \mathcal{O}_{X}$ by coherent locally free $\mathcal{O}_{P}$-modules

$$
0 \longrightarrow \mathcal{F}^{-d} \longrightarrow \cdots \longrightarrow \mathcal{F}^{-1} \longrightarrow \mathcal{F}^{0} \stackrel{\pi}{\longrightarrow} \imath_{*} \mathcal{O}_{X}
$$

where $\mathcal{F}^{0}=\mathcal{O}_{P}$ and $\pi$ is the natural surjection. Since all modules in the above augmented complex are flat over $Y$, therefore $\mathcal{F}^{\bullet}:=q^{*} \mathcal{F}^{\bullet} \stackrel{q^{*} \pi}{\longrightarrow} \imath_{*}^{\prime} \mathcal{O}_{X^{\prime}}$ is a resolution of $\imath_{*}^{\prime} \mathcal{O}_{X^{\prime}}$ by coherent locally free $\mathcal{O}_{P^{\prime}}$-modules, with $\mathcal{F}^{\prime 0}=\mathcal{O}_{P^{\prime}}$. Let

$$
\begin{aligned}
\mathcal{Q}^{\bullet} & =\mathcal{H o m}_{\mathcal{O}_{P}}\left(\mathcal{F}^{\bullet}, \tilde{\omega}_{p}\right), \\
\mathcal{Q}^{\prime \bullet} & =\mathcal{H o m}_{\mathcal{O}_{P^{\prime}}}\left(\mathcal{F}^{\prime \bullet}, \tilde{\omega}_{p^{\prime}}\right) .
\end{aligned}
$$

Using the fact that $\tilde{\omega}_{p^{\prime}}=q^{*} \tilde{\omega}_{p}$, we have a natural map $q^{*} \mathcal{Q} \rightarrow \mathcal{Q}^{\prime \bullet}$ for each $k$, and since $\mathcal{F}^{-k}$ is locally free, this map is an isomorphism. We therefore have an isomorphism of complexes

$$
q^{*} \mathcal{Q}^{\bullet} \stackrel{\sim}{\longrightarrow} \mathcal{Q}^{\bullet} .
$$

Taking the $d$ th cohomology of both sides, we get the isomorphism $\psi_{g}^{f}$. This isomorphism is independent of the resolution $\mathcal{F}^{\bullet}$ of $\imath_{*} \mathcal{O}_{X}$, and is compatible with Zariski localizations of $X$, and hence globalizes. In other words, the map is defined even if $P$ is not quasi-projective over $Y$.

Theorem 2.3.6 asks us to prove that the diagram

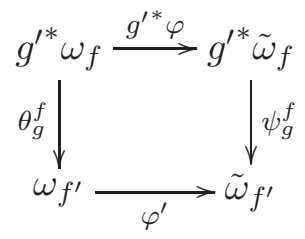

commutes. The rest of $\S 8$ is devoted to proving this.

\subsection{Transitivity for the map $\psi_{g}^{f}$}

The title of this subsection refers to the relations (8.10) and (8.11). The former is an analog of (3.5) and the latter an analog of (3.7). The notation $\tilde{\omega}_{f}^{\Delta}$ and $\psi_{g}^{\Delta}$ is on occasion preferable to $\tilde{\omega}_{f}$ and $\psi_{g}^{f}$, especially if we wish to emphasize the dependence on the commutative triangle $(\Delta)$. To allow a more flexible use of this, for any map $\gamma: Z \rightarrow Y$ let $\left(\Delta_{\gamma}\right)$ denote the base change of the triangle $(\Delta)$ 
by $\gamma$. In the situation of the theorem, suppose we have another map $\tilde{g}: Y^{\prime \prime} \rightarrow Y$, and let

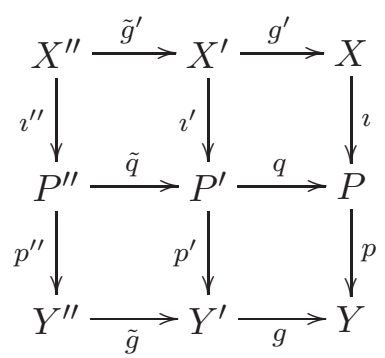

be the resulting commutative diagram. Let $f^{\prime \prime}=p^{\prime \prime} \circ \imath^{\prime \prime}$. Set $\tilde{\omega}_{p^{\prime \prime}}=\Omega_{p^{\prime \prime}}^{n}, \tilde{\omega}_{f^{\prime \prime}}=\tilde{\omega}_{f^{\prime \prime}}^{\Delta} \Delta_{g \tilde{g}}, \psi_{g \tilde{g}}^{f}=\psi_{g \tilde{g}}^{\Delta}$ and $\psi_{\tilde{g}}^{f^{\prime}}=\psi_{\tilde{g}}^{\Delta_{g}}$. Then we check that

$$
\psi_{g \tilde{g}}^{f}=\psi_{\tilde{g}}^{f^{\prime} \circ \tilde{g}^{*} \psi_{g}^{f}} .
$$

In greater detail, assume without loss of generality that $p: P \rightarrow Y$ is quasi-projective, and let $\mathcal{F}^{\bullet}$, $\mathcal{Q}^{\bullet}, \mathcal{F}^{\bullet}, \mathcal{Q}^{\prime \bullet}$ etc. be as in $\S$ 8.1. Setting $\mathcal{F}^{\prime \prime}{ }^{\bullet}=\tilde{q}^{*} \mathcal{F}^{\prime \bullet}=(q \tilde{q})^{*} \mathcal{F}^{\bullet}$ and $\mathcal{Q}^{\prime \prime}=\mathcal{H}_{o m} m_{\mathcal{O}_{P}}\left(\mathcal{F}^{\prime \prime}, \tilde{\omega}_{p^{\prime \prime}}\right)$, the following diagram is checked to commute; the downward arrow on the right arising from the canonical isomorphism $q^{*} \mathcal{Q}^{\bullet} \rightarrow \mathcal{Q}^{\prime \bullet}$, and the one on the left being the one analogous to the following.

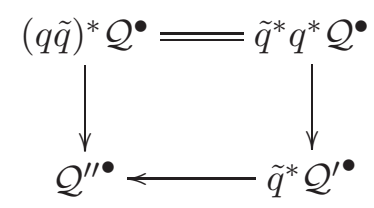

Equation (8.10) follows on taking the $d$ th cohomology.

Next suppose that in addition to $g: Y^{\prime} \rightarrow Y$, we have another base extension map $u: T \rightarrow Y$. Consider the resulting diagram of base changes (3.6). Define $\tilde{\omega}_{f_{T}}:=\tilde{\omega}_{f_{T}}^{\Delta u}, \tilde{\omega}_{f_{T}^{\prime}}:=\tilde{\omega}_{f_{T}^{\prime}}^{\Delta_{u^{\prime}}}, \psi_{u}^{f}:=\psi_{u}^{\Delta}$, $\psi_{u^{\prime}}^{f^{\prime}}:=\psi_{u^{\prime}}^{\Delta_{g}}$ and $\psi_{h}^{f_{T}}:=\psi_{h}^{\Delta_{u}}$. Then, by (8.10) we get

$$
\psi_{h}^{f_{T} \circ h^{\prime *}} \psi_{u}^{f}=\psi_{u^{\prime}}^{f^{\prime} \circ v^{\prime *}} \psi_{g}^{f} .
$$

\subsection{Reduction of the problem}

Since the statement of Theorem 2.3.6 is local on $P$, for the rest of $\S 8$ we will assume without loss of generality that $P$ is quasi-projective, so that $\imath_{*} \mathcal{O}_{X}$ has a resolution of length $r$ by locally free $\mathcal{O}_{P}$-modules. If an intermediate result is local and does not require the hypothesis that $P$ is quasi-projective (e.g. (8.17)) we will point this out.

\subsection{The trace map $T_{\imath}$}

Note that the natural map of complexes (the identity map in degree 0 ), $\mathcal{O}_{P}[0]=\mathcal{F}^{0}[0] \rightarrow \mathcal{F}^{\bullet}$, is compatible with the natural surjection $\mathcal{O}_{P} \longrightarrow \imath_{*} \mathcal{O}_{X}$. In other words, in $D_{\mathrm{qc}}^{b}(P)$ we have a commutative diagram (in which we avoid writing the localization functor $Q_{P}$ for convenience)

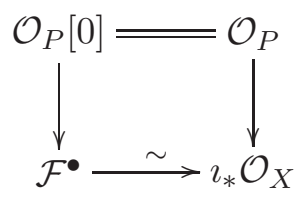




\section{P. SAstry}

the horizontal arrow arising from the resolution $\mathcal{F}^{\bullet} \rightarrow \imath_{*} \mathcal{O}_{X}$. It follows that if $e: R \mathcal{H}^{\circ} m_{\mathcal{O}_{P}}\left(\imath_{*} \mathcal{O}_{P}\right.$, $\left.\tilde{\omega}_{p}\right) \rightarrow R \mathcal{H o m}_{\mathcal{O}_{P}}\left(\mathcal{O}_{P}, \tilde{\omega}_{p}\right)=\tilde{\omega}_{p}$ is 'evaluation at 1', then the diagram

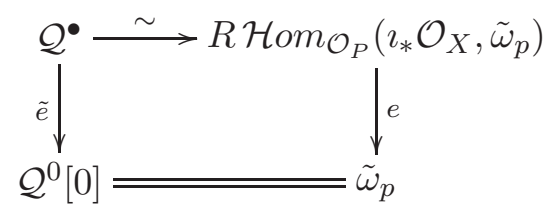

commutes, where $\mathcal{Q}^{\bullet} \stackrel{\tilde{e}}{\longrightarrow} \mathcal{Q}^{0}[0]$ is the natural projection to the 0th degree. We also have a natural surjection $\mathcal{Q}^{d} \rightarrow \operatorname{coker}\left(\mathcal{Q}^{d-1} \rightarrow \mathcal{Q}^{d}\right) \stackrel{\sim}{\longrightarrow} \imath_{*} \tilde{\omega}_{f}$, and this gives a map of complexes

$$
\gamma: \mathcal{Q}^{\bullet} \rightarrow \imath_{*} \tilde{\omega}_{f}[-d]
$$

which is a quasi-isomorphism since $\mathcal{E} x t_{\mathcal{O}_{P}}^{k}\left(\imath_{*} \mathcal{O}_{X}, \tilde{\omega}_{p}\right)=0$ for $k \neq d$. Note that by the definition of $\tilde{\varphi}$ (see (8.3)), the diagram

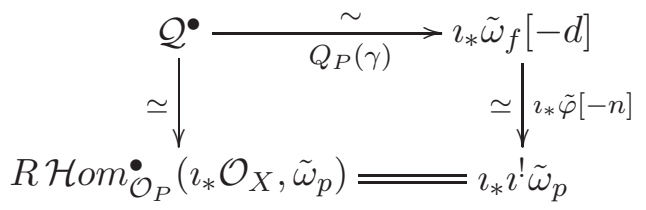

commutes (the equality at the bottom being the identification in (8.1)). From (8.12), (8.13) and the description of the trace $T_{\imath}$ in (8.2), we see that

$$
T_{\imath} \circ \imath_{*} \tilde{\varphi}[-n]=\tilde{e} \circ Q_{P}(\gamma)^{-1} .
$$

If $\tilde{e}^{\prime}: \mathcal{Q}^{\prime \bullet} \rightarrow \mathcal{Q}^{\prime 0}[0]=\tilde{\omega}_{p^{\prime}}$ and $\gamma^{\prime}: \mathcal{Q}^{\prime \bullet} \rightarrow \imath_{*}^{\prime} \tilde{\omega}_{f^{\prime}}[-d]$ are the maps analogous to $\tilde{e}$ and $\gamma$ (for the diagram $\Delta_{g}$ ) then we have

$$
T_{\imath^{\prime} \circ \imath^{\prime}} \tilde{\varphi}^{\prime}[-n]=\tilde{e}^{\prime} \circ Q_{P^{\prime}}\left(\gamma^{\prime}\right)^{-1}
$$

\subsection{The flat base change case}

Suppose $g$ is flat. By definition of $\psi_{g}^{f}$, the following diagram commutes in $D_{\mathrm{qc}}^{+}(P)$.

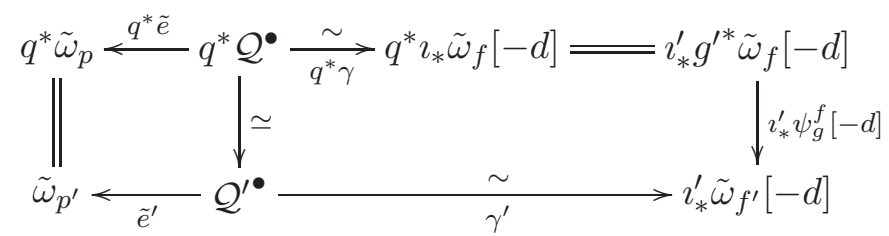

Recall that we have a unique map (in fact an isomorphism) $\Theta_{q}^{\imath}: q^{*} \imath^{!} \stackrel{\sim}{\longrightarrow} \imath^{\prime !} g^{\prime *}$ such that $T_{\imath^{\prime} \circ \imath^{\prime}} \Theta_{\imath}^{q}=$ $q^{*} T_{i}$, where we are identifying $\imath_{*}^{\prime} g^{\prime *}$ with $q^{*} \imath_{*}$ (see (1.6)). The identities (8.14) and (8.15) together with (8.16) give

$$
\Theta_{q}^{\imath}\left(\tilde{\omega}_{p}\right)=\tilde{\varphi}^{\prime}[-n] \circ \psi_{g}^{f}[-d] \circ g^{\prime *} \tilde{\varphi}[-n]^{-1} .
$$

Now Remark B.2.3, together with the concluding sentences of Remark B.2.4, apply to the following commutative diagram.

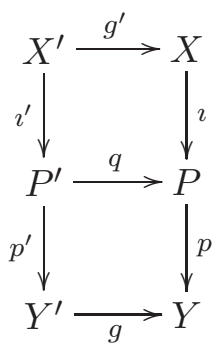


Diagram (B.3), together with the fact that $\Theta_{g}^{p}\left(\mathcal{O}_{Y}[-n]\right)$ is the identity map on $\tilde{\omega}_{p^{\prime}}$, gives us the following commutative diagram.

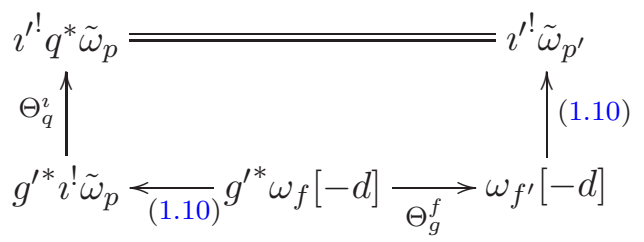

If we use the relation (8.17) together with (8.5) and (8.8) we obtain, from the above commutative diagram, Theorem 2.3.6 when $g$ is flat.

Remark 8.5.1. The relation (8.17) remains true without the quasi-projective hypothesis since the question is local on $P$.

\subsection{Non-flat base change}

We no longer assume that $g$ is flat. We are about to complete the proof of Theorem 2.3.6 in this subsection. We assume, without loss of generality, that $p$ is $n$-compactifiable. This assumption is made so that we may apply Proposition 6.2.2 to the map $p$. We will identify $\omega_{f}$ with $\tilde{\omega}_{f}$ (respectively $\omega_{f^{\prime}}$ with $\left.\tilde{\omega}_{f^{\prime}}\right)$ via $\varphi$ (respectively $\left.\varphi^{\prime}\right)$. We have to show that $\psi_{g}^{f}=\theta_{g}^{f}$.

Suppose $Z \hookrightarrow X$ is a closed immersion which is good for $f$ (this does not imply that the resulting immersion of $Z$ into $P$ is good for $p$ ). Let $\operatorname{res}_{Z}^{f}: R_{Z}^{r} f_{*} \tilde{\omega}_{f} \rightarrow \mathcal{O}_{Y}$ and $\operatorname{res}_{Z}^{p}: R_{Z}^{n} p_{*} \tilde{\omega}_{p} \rightarrow \mathcal{O}_{Y}$ denote the respective residues. If $Z^{\prime}=g^{\prime-1}(Z)$, then in a similar manner we have residue maps $\operatorname{res}_{Z^{\prime}}^{f^{\prime}}$ and $\operatorname{res}_{Z^{\prime}}^{p^{\prime}}$. Let $\bar{p}: \bar{P} \rightarrow Y$ be a compactification of $p$ (not necessarily an $n$-compactification). Let $\bar{\imath}: \bar{X} \hookrightarrow \bar{P}$ be the scheme theoretic closure of $X \hookrightarrow \bar{P}$ and let $\bar{f}: \bar{X} \rightarrow Y$ be the composite $\bar{p} \circ \bar{\imath}$. Identifying $\bar{f}^{!}$ with $\bar{l}^{!} \bar{p}^{!}$via (1.10) we have the trace map $T_{\bar{\imath}}: \bar{\imath}_{*} \bar{f}^{!} \rightarrow \bar{p}^{!}$. Similarly identifying $\tilde{\omega}_{f}[-d]$ with $\imath^{!} \tilde{\omega}_{p}$ via $\tilde{\varphi}[-n]$, which incidentally corresponds to $(1.10)$ by $(8.5)$, we have a map $T_{\imath}\left(\tilde{\omega}_{p}\right): \imath_{*} \tilde{\omega}_{f}[-d] \rightarrow \tilde{\omega}_{p}$. Note that $\tilde{\omega}_{p}=p^{!} \mathcal{O}_{Y}[-n]$ and $\tilde{\omega}_{f}[-d]=f^{!} \mathcal{O}_{Y}[-n]$. We have a commutative diagram as follows.

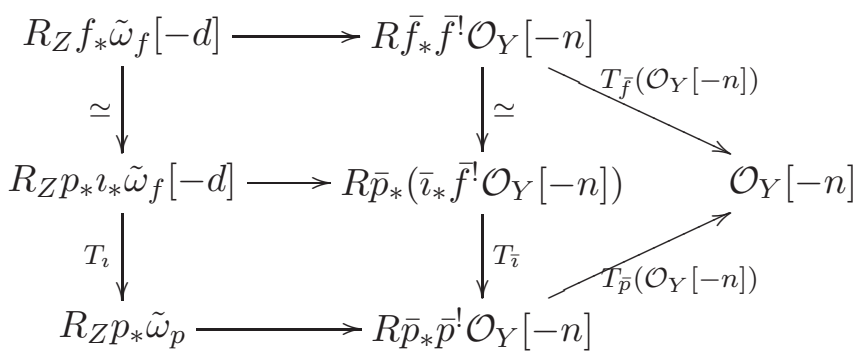

The top horizontal arrow followed by the map $T_{\bar{f}}\left(\mathcal{O}_{Y}[-n]\right)$ is the same as the one obtained by applying the translation functor $(\cdot)[-n]$ to the composite (3.1). The bottom horizontal arrow followed by the map $T_{\bar{p}}\left(\mathcal{O}_{Y}[-n]\right)$ is again $(3.1)[-n]$, this time applied to the map $p$. Taking the $n$th cohomology we get from the definition of residue (3.2) and from the identity (1.4), a commutative diagram

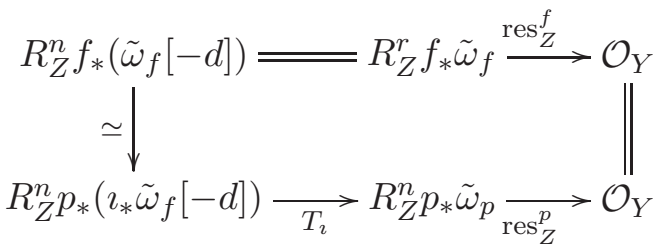

and a similar commutative diagram for the diagram $\Delta_{g}$. The equality on the top left corner is (1.3). 


\section{P. SASTRY}

Consider now the following diagram.

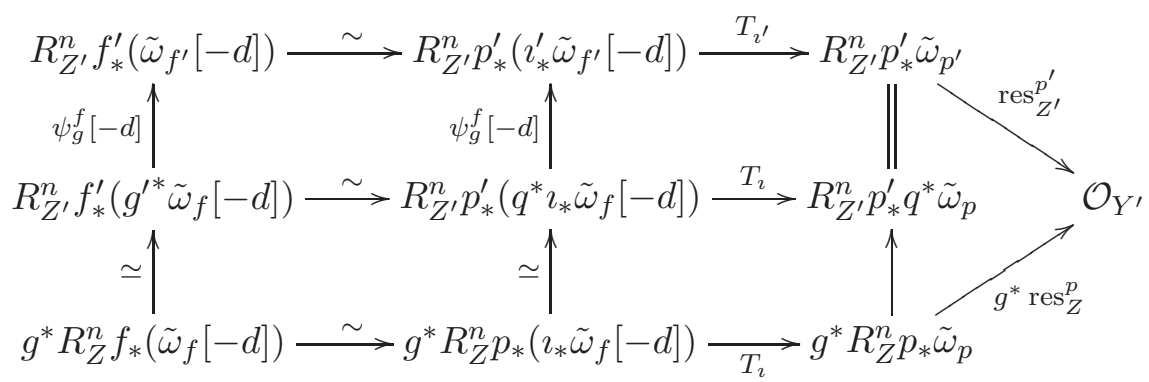

The triangle on the right commutes by $(6.2 .2)$ and this is where we use our hypothesis that $P$ has an $n$-compactification over $Y$. Other subrectangles clearly commute. We thus get, using the commutativity of (8.18) (for the diagram $\Delta$ as well as the diagram $\Delta_{g}$ ) that

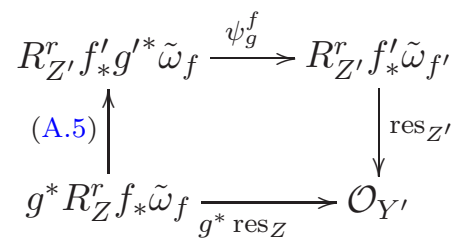

commutes. Since the above diagram also commutes with $\theta_{g}^{f}$ substituted for $\psi_{g}^{f}$ (see Proposition 6.2.2, case c), and since the arrow on the left column is an isomorphism (see Proposition B.1.3) we get by local duality that the completion of $\psi_{g}^{f}$ along $Z^{\prime}$ is equal to the completion of $\theta_{g}^{f}$ along $Z^{\prime}$. In particular, for any point $x^{\prime} \in Z^{\prime}, \hat{\theta}_{g, x^{\prime}}^{f}=\hat{\psi}_{g, x^{\prime}}^{f}$ where $\widehat{(\cdot)}$ denotes completion with respect to the maximal ideal (of variable local rings).

We run into an old problem (see the proof of parts a and b Theorem 2.3.3 in $\S 3.3$ ), namely, we may not have any good immersion for $f$ in the Zariski topology, and we need plenty of them for the above strategy to work. We get around it exactly as before. Pick a point $x \in X$ which is closed in the fiber $f^{-1} f(x)$, set $T$ equal to the spectrum of the completion of the local ring $\mathcal{O}_{Y, f(x)}$, and $u: T \rightarrow Y$ the resulting map. Now use the notations of $\S 8.2$ (and make the identifications $\omega_{f_{T}}=\tilde{\omega}_{f_{T}}, \omega_{f_{T}^{\prime}}=\tilde{\omega}_{f_{T}^{\prime}}$. . As in $\S 3.3$, we can find a good immersion $Z \hookrightarrow S$ for $f_{T}$ such that $Z$ is the spectrum of a complete local ring whose only closed point corresponds to the point $x \in X$. In greater detail, let $k$ be the residue field of $\mathcal{O}_{Y, f(x)}$, and $\bar{x} \in X \times_{Y}$ Spec $k=S \times_{T}$ Spec $k$ the point corresponding to $\bar{x}$. By what we have just proved, $\hat{\theta}_{h, s^{\prime}}^{f_{T}}=\hat{\psi}_{h, s^{\prime}}^{f_{T}}$ for every $s^{\prime} \in h^{\prime-1}(s)$. Since $u$ and $u^{\prime}$ are flat, we have $\theta_{u}^{f}=\psi_{u}^{f}$ and $\theta_{u^{\prime}}^{f_{T}^{\prime}}=\psi_{u^{\prime}}^{f_{T}^{\prime}}$. We also have the identities

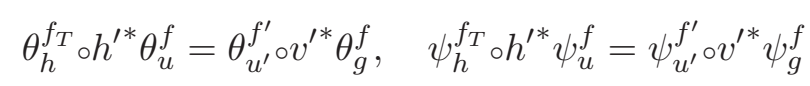

from Remark 3.3.2 and Equation (8.11). We conclude (with $\theta=v^{\prime *} \theta_{g}^{f}$ and $\psi=v^{\prime *} \psi_{g}^{f}$ ) that $\hat{\theta}_{s^{\prime}}=\hat{\psi}_{s^{\prime}}$ for every $s^{\prime} \in h^{\prime-1}(s)$. This is another way of saying that

$$
\hat{\theta}_{g, x^{\prime}}^{f}=\hat{\psi}_{g, x^{\prime}}^{f}
$$

for every $x^{\prime} \in g^{\prime-1}(x)$. Varying $x$ over points of $X$ which are closed in their fiber over $Y$, we see that (8.19) holds for every $x^{\prime} \in X^{\prime}$ which is closed in its fiber over $Y^{\prime}$. This proves Theorem 2.3.6.

\section{ACKNOWLEDGEMENTS}

I am extremely grateful to Brian Conrad for numerous extremely stimulating discussions, for correcting the inaccuracies in my earlier description of his work, for many helpful comments which have 
considerably improved the exposition of the contents of this paper, and for his unfailing professional courtesy. I am very grateful to Joe Lipman for very stimulating discussions and for bringing the work [Con00] to my attention. Thanks are due to Suresh Nayak for mathematical stimulation during the summer of 2000. Ana Jeremías and Leo Alonso patiently went through one of the early versions, corrected typographical errors and mistakes, and I am grateful to them. A large portion of this work was done while I was visiting Purdue University in 2000-2001. This work was partially supported by funds from the GANITA Laboratory at the University of Toronto at Mississauga.

\section{Appendix A. The base change map for higher direct images}

This appendix discusses briefly the definitions and first properties of base change of higher direct images with supports in a closed subset $Z \hookrightarrow X$ for a separated quasi-compact map $f: X \rightarrow Y$ of schemes. We do this along the lines of [GD61b, (1.4.15)] (where the support is $Z=X$ ), and therefore we do not give elaborate proofs. In fact we use [GD61b] to establish that $R_{Z}^{i} f_{*}$ commutes with flat base change (Proposition B.2.1). A general reference for all the appendices is [Gro68]. One motivating reason for these appendices is to compare two versions of the base change map for direct image with supports. These are (a) the map (A.5) and (b) isomorphism (C.10). The first has certain advantages largely summarized in Proposition A.1.1 whereas the second plays a crucial role in proving the compatibility of residues with base change (Proposition 3.2.2). The comparison is achieved in Proposition C.3.1.

\section{A.1 Base change and direct image with supports}

Since much of the paper rests on the base change map for direct image with supports, we give the definition and first properties. Consider a cartesian square

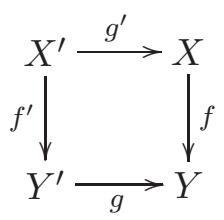

with $f$ separated and quasi-compact. Let $Z \hookrightarrow X$ be a closed subset, and $Z^{\prime}=g^{\prime-1}(Z)$. For every non-negative integer $i$ we have a map

$$
R_{Z}^{i} f_{*} g_{*}^{\prime} \longrightarrow g_{*} R_{Z^{\prime}}^{i} f_{*}^{\prime}
$$

defined as the composite

$$
R_{Z}^{i} f_{*} g_{*}^{\prime} \rightarrow R_{Z^{\prime}}^{i}\left(f \circ g^{\prime}\right)_{*}=R_{Z^{\prime}}^{i}\left(g^{\circ} f^{\prime}\right)_{*} \rightarrow g_{*} R_{Z^{\prime}}^{i} f_{*}^{\prime},
$$

where the two arrows arise from standard spectral sequence $\operatorname{arguments.~Taking~} Z=X$, we get, as a special case, a map $R^{i} f_{*} g^{\prime *} \rightarrow g_{*} R^{i} f_{*}^{\prime}$.

We leave it to the reader to check, from the definitions, the following facts (all unlabeled arrows are either canonical or arise from (A.1) (occasionally with $Z=X)$ ).

i) For each $i$, the diagram

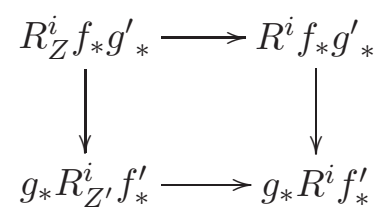

commutes. 


\section{P. SASTRY}

ii) Let $U=X \backslash Z, U^{\prime}=X^{\prime} \backslash Z^{\prime}$. Let $\imath: U \rightarrow X$ and $\imath^{\prime}: U^{\prime} \rightarrow X^{\prime}$ be the resulting open immersions, $g^{\prime \prime}: U^{\prime} \rightarrow U$ the map induced by $g^{\prime}$. For each $i$, we have connecting homomorphisms

$$
R^{i-1}(f \imath)_{*} \imath^{*} \rightarrow R_{Z}^{i} f_{*}, \quad R^{i-1}\left(f^{\prime} \imath^{\prime}\right)_{*} \iota^{\prime *} \rightarrow R_{Z^{\prime}}^{i} f_{*}^{\prime}
$$

arising from the respective excision exact sequences. In greater detail, for $\mathcal{F} \in X_{\mathrm{qc}}$ we have a short exact sequence of complexes

$$
0 \rightarrow f_{*} \circ \underline{\Gamma}_{Z} I^{\bullet} \rightarrow f_{*} I^{\bullet} \rightarrow(f \imath)_{*} \imath^{*} I^{\bullet} \rightarrow 0
$$

where $I^{\bullet}$ is an injective (or even flasque) resolution of $\mathcal{F}$, giving the map

$$
R^{i-1}(f \imath)_{*} \imath^{*} \mathcal{F} \longrightarrow R_{Z}^{i} f_{*} \mathcal{F} .
$$

A similar description applies for the second map. One checks that the diagram

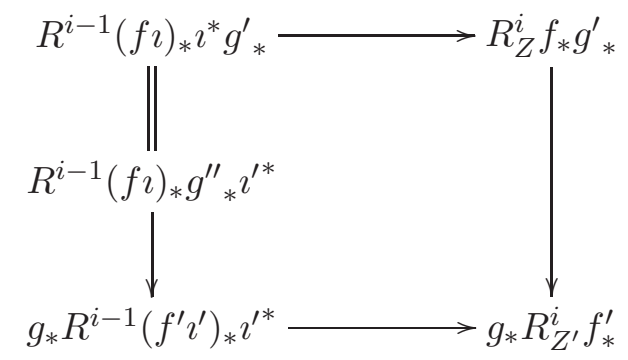

commutes.

Recall that for a map of schemes $h: W^{\prime} \rightarrow W$, we have a natural map $1_{W_{\mathrm{qc}}} \rightarrow h_{*} h^{*}$, making $h^{*}$ the left adjoint of $h_{*}$. Therefore the composite map

$$
R_{Z}^{i} f_{*} \longrightarrow R_{Z}^{i} f_{*} g_{*}^{\prime} g^{\prime *} \longrightarrow g_{*} R_{Z^{\prime}}^{i} f_{*}^{\prime} g^{\prime *}
$$

(with the first arrow arising from $1_{X_{\mathrm{qc}}} \rightarrow g^{\prime}{ }_{*} g^{\prime *}$ ) induces the base change map

$$
g^{*} R_{Z}^{i} f_{*} \longrightarrow R_{Z^{\prime}}^{i} f_{*}^{\prime} g^{\prime *}
$$

(compare with [GD61b, (1.4.15.4)]). As in [GD61b, (1.4.15)], the map (A.5) is compatible with Zariski localizations into $Y$ and $Y^{\prime}$.

The various results above give the following proposition.

Proposition A.1.1. With the above notations the following diagrams commute (with unlabeled arrows being either canonical or arising from (A.5)):

a)

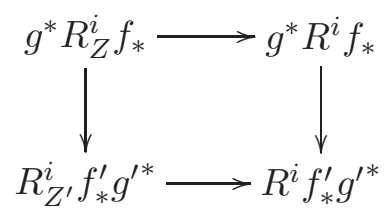

b)

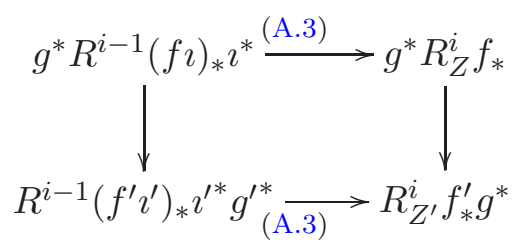

Moreover, all these diagrams behave well with respect to Zariski localizations into $Y$ or $Y^{\prime}$. 


\section{Base Change For COHEN-MaCAulay MaPs}

\section{Appendix B. Isomorphism theorems for the base change map}

This appendix recalls statements (and proofs) concerning conditions under which (A.5) is an isomorphism. Most of these can be deduced easily from discussions in [GD64, $\S$ (1.4), especially (1.4.15) and (1.4.16)]. Our approach is minimalistic, we only prove the results needed in this paper and (reluctantly) eschew the temptation to give the most general statement we could think of. We use the same notations $\left(f, f^{\prime}, g, g^{\prime}, Z, Z^{\prime}\right.$ etc.) and hypotheses as we did in Appendix A.

\section{B.1 Highest direct image}

The result that is most critical for the definition of $\theta_{g}^{f}$ (and perhaps the best known in the non-flat base change situation) is Proposition B.1.2. We supply a proof because we do not assume $f$ is proper. This relaxation is important in the proof of Proposition B.1.3, without which we cannot even state results concerning base change for residues along good immersions (Proposition 3.2.2).

Remark B.1.1. Our convention for the map from the Čech to the derived functor theory is this (with notations as in the proposition above): Let $\mathcal{C}=\mathcal{C} \bullet(\mathfrak{U}, \mathcal{F})$ be the sheaf Cech complex associated to $(\mathfrak{U}, \mathcal{F}), \mathcal{I}^{\bullet}$, and $\varphi: \mathcal{C} \rightarrow \mathcal{I}^{\bullet}$ the homotopy unique map lifting the identity map on $\mathcal{F}$ (recall, $\mathcal{C}$ is a resolution of $\mathcal{F})$. Then the downward arrow on the left of diagram (B.1) is $H^{i}(\Gamma(X, \varphi))$. It is with this convention that the diagram (B.1) commutes.

Proposition B.1.2. If $R^{k} f_{*}=0=R^{k} f_{*}^{\prime}$ for $k>r$, then the base change map

$$
g^{*} R^{r} f_{*} \longrightarrow R^{r} f_{*}^{\prime} g^{*}
$$

is an isomorphism.

Proof. Since the base change map (A.5) is compatible with open immersions into $Y$ and $Y^{\prime}$, we may assume that $Y=\operatorname{Spec} A$ and $Y^{\prime}=\operatorname{Spec} A^{\prime}$. According to the discussion in the proof of [GD64, (1.4.15)] (especially towards the end), if $\mathfrak{U}$ is a finite affine open cover of $X, \mathcal{F}$ a quasi-coherent $\mathcal{O}_{X}$-module, $\mathcal{F}^{\prime}=g^{\prime *} \mathcal{F}, \mathfrak{U}^{\prime}=g^{\prime-1}(\mathfrak{U})$ (the notation being obvious), then the diagram

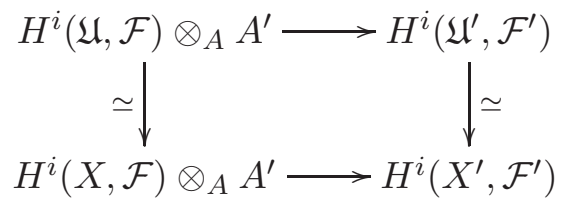

commutes, where the following hold.

- The downward arrows are the canonical maps (in this case isomorphisms, since $\mathfrak{U}$ and $\mathfrak{U}^{\prime}$ are affine covers on separated schemes) comparing Čech cohomology to the derived functor cohomology (see Remark B.1.1).

- The top horizontal arrow is the natural map

$$
H^{i}\left(C^{\bullet}(\mathfrak{U}, \mathcal{F})\right) \otimes_{A} A^{\prime} \longrightarrow H^{i}\left(C^{\bullet}(\mathfrak{U}, \mathcal{F}) \otimes_{A} A^{\prime}\right) .
$$

We are using the fact that $C^{\bullet}\left(\mathfrak{U}^{\prime}, \mathcal{F}^{\prime}\right)=C^{\bullet}(\mathfrak{U}, \mathcal{F}) \otimes_{A} A^{\prime}$.

- The bottom horizontal arrow is (the global sections of) our base change map (A.5).

So we are reduced to showing that

$$
H^{r}(\mathfrak{U}, \mathcal{F}) \otimes_{A} A^{\prime} \longrightarrow H^{r}\left(\mathfrak{U}^{\prime}, \mathcal{F}^{\prime}\right)
$$

is an isomorphism. Next, if $M$ is an $A$-module, $\mathcal{F}_{M}$ the $\mathcal{O}_{X}$-module $\mathcal{F} \otimes f^{*} \widetilde{M}$, we have, using $C^{\bullet}\left(\mathfrak{U}, \mathcal{F}_{M}\right)=C^{\bullet}(\mathfrak{U}, \mathcal{F}) \otimes_{A} M$, a map (functorial in $\left.M\right) \psi_{M}: H^{r}(\mathfrak{U}, \mathcal{F}) \otimes_{A} M \rightarrow H^{r}\left(\mathfrak{U}, \mathcal{F}_{M}\right)$ which specializes to (B.2) when $M=A^{\prime}$. Now $\psi$ is a map of right exact functors on the category of 


\section{P. SASTRY}

$A$-modules, and it is an isomorphism on free $A$-modules. Therefore it is an isomorphism on all $A$ modules. We are using the fact that tensor products and Čech cohomology commute with arbitrary direct limits (to see that it is an isomorphism for free $A$-modules of arbitrary rank).

Proposition B.1.3. Suppose $f: X \rightarrow Y$ is Cohen-Macaulay of relative dimension $r$, and $\imath: Z \hookrightarrow X$ a good immersion for $f$ (see Definition 3.1.4). Then

$$
g^{*} R_{Z}^{r} f_{*} \longrightarrow R_{Z^{\prime}}^{r} f_{*}^{\prime} g^{\prime *}
$$

is an isomorphism.

Proof. Again, we may assume $Y=\operatorname{Spec} A$ and $Y^{\prime}=\operatorname{Spec} A^{\prime}$. In fact, by the definition of good immersion, we may choose $A$ in such a way that $Z$ has an affine open neighborhood $\operatorname{Spec} R$ such that $Z$ is given by the vanishing of a Koszul-regular $R$-sequence $\boldsymbol{t}=\left(t_{1}, \ldots, t_{r}\right)$. By excision and the nature of the proposition we wish to prove, we may assume that $X=\operatorname{Spec} R$. If $U_{i}=\operatorname{Spec} R_{t_{i}}$, then $\mathfrak{U}=\left\{U_{i}\right\}$ is an affine open cover of $X^{*}=X \backslash Z$. Hence $H^{k}\left(X^{*}, \cdot\right)=0$ for $k>r-1$. The same argument shows that $H^{k}\left(X^{*} \otimes_{A} A^{\prime}, \cdot\right)=0$ for $k>r-1$. By the previous proposition $g^{*} R^{r-1}(f \imath)_{*} \imath^{*} \longrightarrow R^{r-1}\left(f^{\prime} \imath^{\prime}\right)_{*} \iota^{*} g^{\prime *}$ is an isomorphism. Moreover, $R^{k} f_{*}=R^{k} f_{*}^{\prime}=0$ for $k \geqslant 1$. If $r>0$ then by Proposition A.1.1 we have a commutative diagram

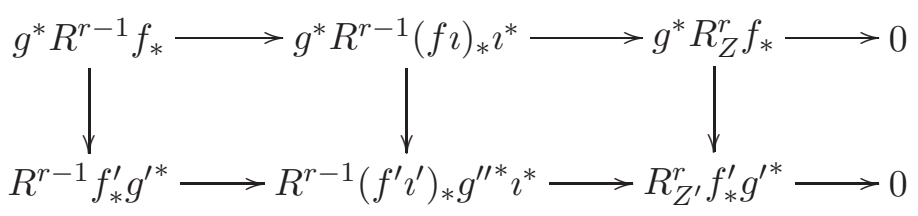

with exact rows (by the right exactness $g^{*}$ ). The left and the middle downward arrows are isomorphisms and hence by the 3-Lemma we are done (if $r>1$ we do not even need the 3-Lemma, for then $\left.R^{r-1} f_{*}=R^{r-1} f_{*}^{\prime}=0\right)$. We leave the case $r=0$ to the reader.

\section{B.2 Flat base change}

The results of $[\mathrm{GD} 61 \mathrm{~b},(1.4 .15)]$ extend obviously to the map (A.5) for flat $g$. We supply a short proof for the record (and for completeness).

Proposition B.2.1. With the notations of the last subsection, if $g$ is flat, then the map $g^{*} R_{Z}^{i} \rightarrow$ $R_{Z^{\prime}}^{i} f_{*}^{\prime} g^{\prime *}$ is an isomorphism for every $i$.

Proof. We have two long exact sequences (arising from (A.2)), one for $(f, Z)$ and another for $\left(f^{\prime}, Z^{\prime}\right)$, and the two are related by the commutative diagram

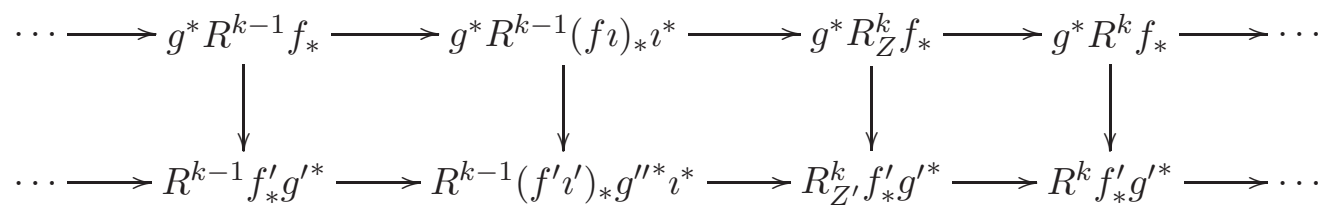

Note that the first row is exact because $g$ is flat. The diagram is commutative by Proposition A.1.1. By [GD61b, (1.4.15)], the downward arrows not involving $Z$ and $Z^{\prime}$ are isomorphisms. The conclusion follows.

Remark B.2.2. One implication of the above proposition is this: if $g$ is flat and $\mathcal{F}$ is flasque, then $g^{\prime *} \mathcal{F}$ is $f_{*} \circ \underline{\Gamma}_{Z^{\prime}}$-acyclic. Therefore (by considering injective resolutions of bounded below complexes and using $\left.g^{*}=L g^{*}, g^{\prime *}=L g^{\prime *}\right)$ we get an isomorphism

$$
L g^{*} R_{Z} f_{*} \stackrel{\sim}{\longrightarrow} R_{Z^{\prime}} f_{*}^{\prime} L g^{\prime *} .
$$


This can also be described (as a map and without assuming $g$ is flat) as the one induced by the adjoint pair $\left(L g^{*}, R g_{*}\right)$ and the composite map

$$
R_{Z} f_{*} \rightarrow R_{Z} f_{*} \circ R g_{*}^{\prime} L g^{\prime *} \stackrel{\sim}{\longrightarrow} R_{Z^{\prime}}\left(f g^{\prime}\right)_{*} L g^{\prime *}=R_{Z^{\prime}}\left(g f^{\prime}\right)_{*} L g^{\prime *} \stackrel{\sim}{\longrightarrow} R g_{*} R_{Z^{\prime}} f_{*}^{\prime} L g^{\prime *}
$$

(the first map coming from the adjoint pair $\left(L g^{\prime *}, R g_{*}^{\prime}\right)$ ). As in Proposition A.1.1, part a, the diagram

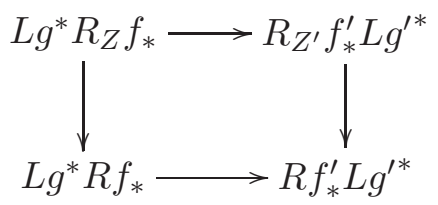

commutes.

Remark B.2.3. With $g$ flat as before, if

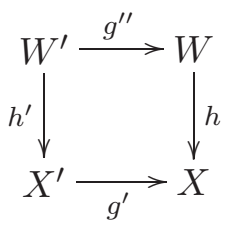

is a second cartesian square, then we check that the diagram

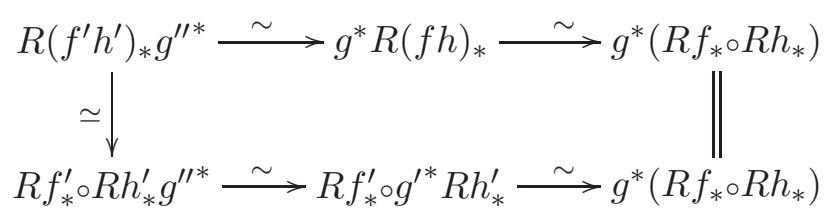

commutes. Details can be found in the proof of [Lip03, p. 112, Proposition (3.7.2)(ii)], especially the diagram (3.7.2.2) on p. 115 in the proof. If $f$ and $h$ are proper, then we see, using the above diagram, that

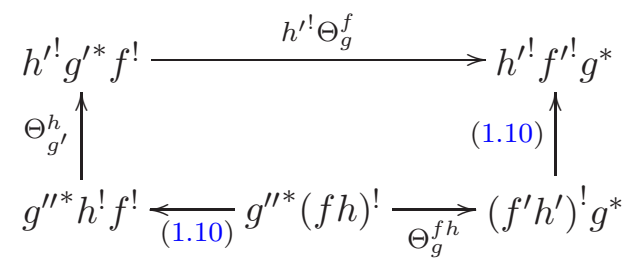

commutes.

Remark B.2.4. Suppose we have a commutative diagram

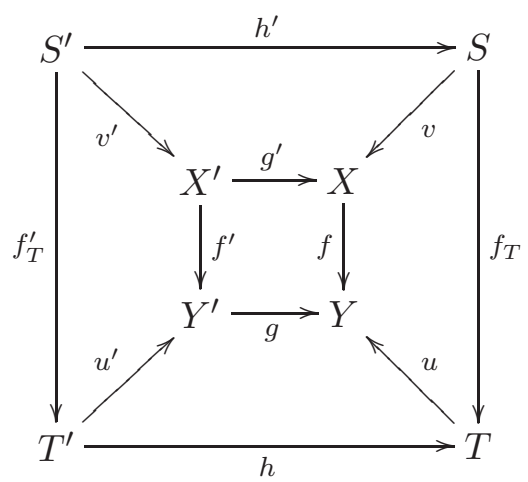

with the two squares and the four trapeziums squeezed between them cartesian and with the only assumption of $f$ being that it is proper. However, assume that $g$ and $u$ are flat. Remark B.2.3 above 


\section{P. SASTRY}

gives us the relation

$$
\Theta_{h}^{f_{T}} \circ h^{\prime *} \Theta_{u}^{f}=\Theta_{u^{\prime}}^{f^{\prime} \circ v^{\prime *}} \Theta_{g}^{f} .
$$

Note that this and the previous remark complete the proof of the assertions made in Remark 1.3.2. In particular, this means that for a general separated finite type map $f$ and a flat map $g$, and $\bar{f}$ a compactification of $f$, then $\Theta_{g}^{\bar{f}} \mid X$ is independent of the compactification $\bar{f}$ (see diagram (1.11)) of $f$. Hence $\Theta_{g}^{f}$ is well defined, and one sees that the diagram (B.3) continues to commute and the equation (B.4) continues to hold even if we drop the properness hypotheses on the various maps for which we imposed the properness condition.

\section{Appendix C. Generalized fractions; Koszul and Čech complexes}

This appendix begins with a collection of well-known results concerning the relationship between local cohomology, Koszul complexes and Čech complexes. The aim is to make explicit the base change map. As before, we recount only as much as we need. Generalized fractions provide a convenient framework for making matters explicit.

\section{C.1 Čech complexes and local cohomology}

Let $R$ be a Noetherian ring, $M$ an $R$-module $I \subset R$ an ideal generated by $\boldsymbol{t}=\left(t_{1}, \ldots, t_{r}\right), X=$ $\operatorname{Spec} R, Z=\operatorname{Spec} R / I, U=X \backslash Z, U_{i}=\operatorname{Spec} R_{t_{i}}, i=1, \ldots, r$, and $\mathfrak{U}=\left\{U_{i}\right\}$, so $\mathfrak{U}$ is an affine open cover of $U$. We have a composite map

$$
M_{t_{1} \ldots t_{r}}=C^{r-1}(\mathfrak{U}, \widetilde{M}) \longrightarrow H^{r-1}(\mathfrak{U}, \widetilde{M}) \stackrel{\sim}{\longrightarrow} H^{r-1}(U, \widetilde{M}) \longrightarrow H_{Z}^{r}(X, \widetilde{M}),
$$

where the map $C^{r-1}(\mathfrak{U}, \widetilde{M}) \rightarrow H^{r-1}(\mathfrak{U}, \widetilde{M})$ arises from the fact that $C^{k}(\mathfrak{U}, \widetilde{M})$ vanishes for $k>r-1$, and hence is surjective. Since $X$ is affine, the connecting map $H^{r-1}(U, \widetilde{M}) \rightarrow H_{Z}^{r}(X, \widetilde{M})$ is an isomorphism if $r>1$ and surjective when $r=1$. In any case, the map (C.1) is a surjective map, and hence elements of $H_{Z}^{r}(X, \widetilde{M})$ can be described as images of 'fractions' in $M_{t_{1} \ldots t_{r}}$. The image of $m / t_{1}^{\alpha_{1}} \ldots t_{r}^{\alpha_{r}} \in M_{t_{1} \ldots t_{r}}$ in $H_{Z}^{r}(X, \widetilde{M})$ is denoted by the generalized fraction

$$
\left[\begin{array}{c}
m \\
t_{1}^{\alpha_{1}}, \ldots, t_{r}^{\alpha_{r}}
\end{array}\right] \text {. }
$$

(In [Lip84], this is denoted $m / \boldsymbol{t}^{\alpha}$.) We refer to [Lip84, p. 60, (7.2)] for the calculus of generalized fractions, especially the 'change of parameters' formula (7.2)(b). While [Lip84] deals with varieties over a perfect field, the change of parameters formula remains valid in the present generality, with exactly the same proof as in [Lip84].

Next, for a sequence of positive integers $\alpha=\left(\alpha_{1}, \ldots, \alpha_{r}\right)$, let $\boldsymbol{t}^{\alpha}$ denote the sequence $\left(t_{1}^{\alpha_{1}}, \ldots, t_{r}^{\alpha_{r}}\right)$ and let $B_{\alpha}=R / \boldsymbol{t}^{\alpha} R$. For an $R$-module $M$, we define (as is standard in commutative algebra)

$$
\Gamma_{I}(M):=\underset{\alpha}{\lim _{\alpha}} \operatorname{Hom}_{R}\left(B_{\alpha}, M\right)=\bigcup_{\alpha}\left(0: \boldsymbol{t}^{\alpha}\right) \subset M .
$$

Let $H^{i}\left(\boldsymbol{t}^{\alpha}, M\right)=H^{i}\left(K^{\bullet}\left(\boldsymbol{t}^{\alpha}, M\right)\right)$. The last two maps in the series of isomorphisms in (4.2) give

$$
H^{i}\left(\boldsymbol{t}^{\alpha}, M\right) \stackrel{\sim}{\longrightarrow} \operatorname{Ext}_{R}^{i}\left(B_{\alpha}, M\right)
$$

giving the well-known isomorphism

$$
\underset{\alpha}{\lim _{\alpha}} H^{i}\left(\boldsymbol{t}^{\alpha}, M\right) \stackrel{\sim}{\longrightarrow} H_{I}^{i}(M) .
$$

Let $K_{\boldsymbol{t}}^{\bullet}=\underset{\alpha}{\lim } K^{\bullet}\left(\boldsymbol{t}^{\alpha}, R\right)$ and $K_{\boldsymbol{t}}^{\bullet}(M)=\underset{\alpha}{\lim } K^{\bullet}\left(\boldsymbol{t}^{\alpha}, M\right)$. Set

$$
H_{\boldsymbol{t}}^{i}(M)=H^{i}\left(K_{\boldsymbol{t}}^{\bullet}(M)\right) \text {. }
$$




\section{BASE CHANGE FOR COHEN-MACAUlay MAPS}

Now $K^{\bullet}\left(\boldsymbol{t}^{\alpha}, M\right)=K^{\bullet}\left(\boldsymbol{t}^{\alpha}, R\right) \otimes_{R} M$. Since tensor product commutes with direct limits, we have $K_{\boldsymbol{t}}^{\bullet} \otimes_{R} M=K_{\boldsymbol{t}}^{\bullet}(M)$. Since cohomology commutes with taking direct limits, the isomorphism (C.3) becomes

$$
H_{\boldsymbol{t}}^{i}(M) \stackrel{\sim}{\longrightarrow} H_{I}^{i}(M) .
$$

The complex $K_{\boldsymbol{t}}^{\bullet}(M)$ is zero in degrees greater than $r$. Hence we have a surjective map $M_{t_{1} \ldots t_{r}}=$ $K_{t}^{r}(M) \rightarrow H_{t}^{r}(M)$. Following this with the isomorphism (C.4) we get a surjective map

$$
M_{t_{1} \ldots t_{r}} \longrightarrow H_{I}^{r}(M)=H_{Z}^{r}(X, \widetilde{M}) .
$$

Recall that, for $i \geqslant 1, K_{\boldsymbol{t}}^{i}(M)=C^{i-1}(\mathfrak{U}, \widetilde{M})$. If $\delta$ and $d$ are the differentials of $K_{\boldsymbol{t}}^{\bullet}(M)$ and $C^{\bullet}(\mathfrak{U}, \widetilde{M})$, then we check that for $p \geqslant 1, \delta^{p}=d^{p-1}$. It follows that we have a surjective map (equality if $r>1$ !)

$$
\varphi: H^{r-1}(\mathfrak{U}, \widetilde{M}) \rightarrow H_{\boldsymbol{t}}^{r}(M) .
$$

We check that the following diagram commutes (the sign on the downward arrow on the left was unfortunately missed in [Lip84, p. 61, (7.2.1)]; see also [LS92, p. 115, (3.4)] where the same error is perpetuated).

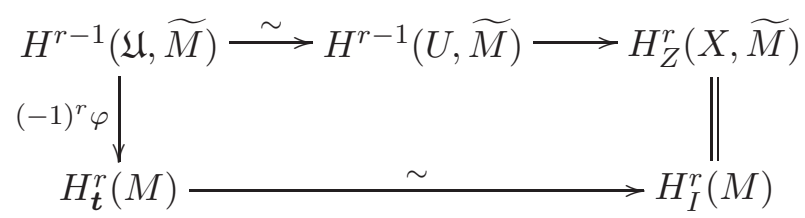

This means that the image of $m / t_{1}^{\alpha_{1}} \cdots t_{r}^{\alpha_{r}}$ under (C.5) is

$$
(-1)^{r}\left[\begin{array}{c}
m \\
t_{1}^{\alpha_{1}}, \ldots, t_{r}^{\alpha_{r}}
\end{array}\right] \text {. }
$$

The reader is urged to experiment with $r=1$. We point out that in our convention if $0 \rightarrow A^{\bullet} \rightarrow$ $B^{\bullet} \rightarrow C^{\bullet} \rightarrow 0$ is a short exact sequence of complexes of abelian groups, then the connecting map $H^{i}\left(C^{\bullet}\right) \rightarrow H^{i+1}\left(A^{\bullet}\right)$ sends $[\gamma]$ to $\left[\mathrm{d}_{B} \beta\right]$, where (a) $\beta \in B^{i}$ is a pre-image of $\gamma \in C^{i}$ and (b) $\mathrm{d}_{B} \beta$ is identified with an $(i+1)$-cocycle in $A^{\bullet}$, since the image of $\mathrm{d}_{B} \beta$ in $C^{i+1}$ is 0 . This convention (without signs) is dictated by the proof of [Lip84, Lemma (8.6), pp. 79-81], a crucial ingredient in the proof of the residue theorem for projective spaces [Lip84, Proposition (8.5)].

Remark C.1.1. Let $\varphi_{1}^{\alpha}: M \rightarrow H_{I}^{r}(M)$ and $\varphi_{2}^{\alpha}: M / \boldsymbol{t}^{\alpha} M \rightarrow H_{I}^{r}(M)$ be the composite maps

$$
\begin{gathered}
M=K^{r}\left(\boldsymbol{t}^{\alpha}, M\right) \rightarrow K_{\boldsymbol{t}}^{r}(M) \stackrel{(\mathrm{C} .5)}{\longrightarrow} H_{I}^{r}(M) \\
M / \boldsymbol{t}^{\alpha} M=H^{r}\left(\boldsymbol{t}^{\alpha}, M\right) \rightarrow H_{\boldsymbol{t}}^{r}(M) \underset{(\mathrm{C} .4)}{\longrightarrow} H_{I}^{r}(M)
\end{gathered}
$$

(the first arrows in each row being the canonical maps to the direct limit over $\alpha$ ). Then in view of diagram (C.6) we have

$$
\varphi_{1}^{\alpha}(m)=(-1)^{r}\left[\begin{array}{l}
m \\
\boldsymbol{t}^{\alpha}
\end{array}\right]=\varphi_{2}^{\alpha}\left(m+\boldsymbol{t}^{\alpha} M\right)
$$

\section{C.2 Koszul complexes and local cohomology}

We retain the notations of $\S$ C.1. We recall and explicate the well-known isomorphism

$$
H_{I}^{r}(R) \otimes_{R} M \stackrel{\sim}{\longrightarrow} H_{I}^{r}(M) .
$$

This isomorphism is crucial to local duality. For each $m \in M$, let $f_{m}: R \rightarrow M$ be the $R$-map $r \mapsto r m$, and $\varphi_{m}: H_{I}^{r}(R) \rightarrow H_{I}^{r}(M)$ the $R$-map induced by $f_{m}$. The form $\psi(\theta, m):=\varphi_{m}(\theta)$ is $R$-bilinear in $\theta \in H_{I}^{r}(R)$ and $m \in M$. This defines (C.7). The map (C.7) is an isomorphism since it is a functorial map in $M$ which is an isomorphism when $M=R$ and since the two functors 


\section{P. SASTRY}

$H_{I}^{r}(\cdot)$ and $H_{I}^{r}(R) \otimes(\cdot)$ are right exact. The same process applied to the functor $H_{\boldsymbol{t}}^{r}(\cdot)$ gives us an isomorphism

$$
H_{\boldsymbol{t}}^{r}(R) \otimes_{R} M \stackrel{\sim}{\longrightarrow} H_{\boldsymbol{t}}^{r}(M) .
$$

The functoriality of the map (C.4) gives the commutativity of the following diagram.

$$
\begin{aligned}
& H_{\boldsymbol{t}}^{r}(R) \otimes_{R} M \stackrel{(\mathrm{C} .8)}{\longrightarrow} H_{\boldsymbol{t}}^{r}(M) \\
& \quad(\mathrm{C} .4) \downarrow \\
& \simeq \quad \simeq \mid(\mathrm{C} .4) \\
& H_{I}^{r}(R) \otimes_{R} M \underset{(\mathrm{C} .7)}{\longrightarrow} H_{I}^{r}(M)
\end{aligned}
$$

Note that the map (C.8) is the natural map $H^{r}\left(K_{\boldsymbol{t}}^{\bullet}\right) \otimes_{R} M \rightarrow H^{r}\left(K_{\boldsymbol{t}}^{\bullet} \otimes_{R} M\right)$, we are using the relation $K_{\boldsymbol{t}}^{\bullet}(M)=K_{\boldsymbol{t}}^{\bullet} \otimes_{R} M$.

Remark C.2.1. In view of the commutative diagram (C.9), the map (C.7) is explicitly described by

$$
\left[\begin{array}{c}
s \\
\boldsymbol{t}^{\alpha}
\end{array}\right] \otimes m \mapsto\left[\begin{array}{c}
s m \\
\boldsymbol{t}^{\alpha}
\end{array}\right]
$$

If $R \rightarrow R^{\prime}$ is an $R$-algebra, with $R^{\prime}$ Noetherian, $\boldsymbol{t}^{\prime}=\left(t_{1}^{\prime}, \ldots, t_{r}^{\prime}\right)$ the image of $\boldsymbol{t}$ in $R^{\prime}$ (so that $I R^{\prime}$ is generated by $\boldsymbol{t}^{\prime}$ ), and $M^{\prime}=M \otimes_{R} R^{\prime}$, then we clearly have

$$
K_{\boldsymbol{t}}^{\bullet}(M) \otimes_{R} R^{\prime}=K_{\boldsymbol{t}^{\prime}}^{\bullet}\left(M^{\prime}\right),
$$

giving an isomorphism (since $r$ is the highest degree of $K_{\boldsymbol{t}}^{\bullet}(M)$ and $K_{\boldsymbol{t}^{\prime}}^{\bullet}\left(M^{\prime}\right)$ )

$$
H_{\boldsymbol{t}}^{r}(M) \otimes_{R} R^{\prime} \stackrel{\sim}{\longrightarrow} H_{\boldsymbol{t}^{\prime}}^{r}\left(M^{\prime}\right)
$$

and this gives an isomorphism (via (C.4))

$$
H_{I}^{r}(M) \otimes_{R} R^{\prime} \stackrel{\sim}{\longrightarrow} H_{I R^{\prime}}^{r}\left(M^{\prime}\right)
$$

given by

$$
\left[\begin{array}{c}
m \\
t_{1}^{\alpha_{1}}, \ldots, t_{r}^{\alpha_{r}}
\end{array}\right] \otimes s \mapsto\left[\begin{array}{c}
m \otimes s \\
t_{1}^{\alpha_{1}}, \ldots, t_{r}^{\alpha_{r}}
\end{array}\right] .
$$

Using the formulae comparing generalized fractions with different sets of generators of $I$ in $\S 7$ of [Lip84], we see that (C.10) does not depend on the generators $\boldsymbol{t}$ of $I$. We can, as we did for (C.7), give an a priori interpretation of the map (C.10) (without using Koszul complexes). In greater detail, for each $r^{\prime} \in R^{\prime}$ we have a map $M \rightarrow M^{\prime}$ given by $m \mapsto m \otimes r^{\prime}$. This induces a composite map $\phi_{r^{\prime}}: H_{I}^{r}(M) \rightarrow H_{I}^{r}\left(M^{\prime}\right) \rightarrow H_{I R^{\prime}}^{r}\left(M^{\prime}\right)$. It can be checked that the map (C.10) is given by $\theta \otimes r^{\prime} \mapsto \phi_{r^{\prime}}(\theta)$ for $\theta \in H_{I}^{r}(M)$ and $r^{\prime} \in R^{\prime}$. The crucial task is to show that for an $R^{\prime}$-module $N$, the identity $H_{\boldsymbol{t}}^{r}(N)=H_{\boldsymbol{t}^{\prime}}^{r}(N)$ corresponds to the map $H_{I}^{r}(N) \rightarrow H_{I R^{\prime}}^{r}(N)$ under (C.5). We give a proof of this in a special case of interest to us, namely, the case where $R$ is a Cohen-Macaulay algebra over a Noetherian ring $A$ and $R^{\prime}=R \otimes_{A} A^{\prime}$ for a Noetherian $A$-algebra $A^{\prime}$. (See Proposition C.3.1.)

Remark C.2.2. If $\widehat{R}$ is the completion of $R$ with respect to $I$, then the natural map $H_{I}^{r}(M) \rightarrow$ $H_{I}^{r}(M) \otimes_{R} \widehat{R}$ is an isomorphism. Using (C.10) this gives us the well-known identification $H_{\widehat{I}}^{r}(\widehat{M})=$ $H_{I}^{r}(M)$ for every finitely generated $R$-module $M$. 


\section{BASE CHANGE FOR COHEN-MACAUlay MAPS}

\section{C.3 Explicit version}

Let $R, t, Z$ etc. be as above. For the rest of this appendix (including $\S$ C.4), let us suppose we have a diagram of Noetherian rings

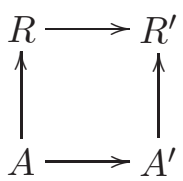

with $R^{\prime}=R \otimes_{A} A^{\prime}$ (and the arrows compatible with this tensor product), and $A \longrightarrow R$ a CohenMacaulay map of relative dimension $r$, i.e. the corresponding map of schemes $f: X=\operatorname{Spec} R \rightarrow$ Spec $A=Y$ is Cohen-Macaulay of relative dimension $r$. Suppose further that $Z \hookrightarrow X$ is a good immersion for $f$ (cf. 3.1.4) and the image of $\boldsymbol{t}$ in $R^{\prime}$ is $\boldsymbol{t}^{\prime}=\left(t_{1}^{\prime}, \ldots, t_{r}^{\prime}\right)$. Set $M^{\prime}=M \otimes_{R} R^{\prime}$. Recall that in this situation (A.5) is an isomorphism by Proposition B.1.3.

Proposition C.3.1. The isomorphism

$$
H_{I}^{r}(M) \otimes_{R} R^{\prime} \stackrel{(\mathrm{A} .5)}{\longrightarrow} H_{I R^{\prime}}^{r}\left(M^{\prime}\right)
$$

is given by

$$
\left[\begin{array}{c}
m \\
t_{1}^{\alpha_{1}}, \ldots, t_{r}^{\alpha_{r}}
\end{array}\right] \otimes s \mapsto\left[\begin{array}{c}
m \otimes s \\
t_{1}^{\prime \alpha_{1}}, \ldots, t_{r}^{\prime \alpha_{r}}
\end{array}\right],
$$

where $m \in M, s \in R^{\prime}$ and $\left(\alpha_{1}, \ldots, \alpha_{r}\right)$ a sequence of positive integers. In other words, the base change isomorphism (A.5) is locally a special case of the isomorphism (C.10), when $f$ is separated Cohen-Macaulay and $Z$ is a good immersion.

Proof. This follows by putting together Proposition A.1.1, part b and diagram (B.1) (for $i=r$ and $i=r-1$, respectively) and using the fact that the map (C.1) is surjective.

\section{C.4 Normal bundles and local cohomology}

With the situation as before, and $\alpha$ as usual a sequence of positive integers, let $N_{\alpha}=N_{B_{\alpha} / R}=$ $\bigwedge_{B_{\alpha}}^{r} \operatorname{Hom}_{B_{\alpha}}\left(I_{\alpha} / I_{\alpha}^{2}, B_{\alpha}\right), I_{\alpha}=\boldsymbol{t}^{\alpha} R$. Let $B_{\alpha}^{\prime}=R^{\prime} / \boldsymbol{t}_{\alpha}^{\prime} R^{\prime}=B_{\alpha} \otimes_{R} R^{\prime}$ and let $N_{\alpha}^{\prime}=N_{B_{\alpha}^{\prime}} / R^{\prime}$. Note that $N_{\alpha}^{\prime}=N_{\alpha} \otimes_{R} R^{\prime}=N_{\alpha} \otimes_{A} A^{\prime}$.

Now, for $\alpha \leqslant \alpha^{\prime}$ (i.e. $\alpha_{i} \leqslant \alpha^{\prime}{ }_{i}$ for $i=1, \ldots, r$ ) we have a map $N_{\alpha} \rightarrow N_{\alpha^{\prime}}$ given by

$$
\frac{1}{\left(t_{1}^{\alpha_{1}}, \ldots, t_{r}^{\alpha_{r}}\right)} \mapsto \frac{t_{1}^{\beta_{1}} \cdots t_{r}^{\beta_{r}}}{\left(t_{1}^{\alpha_{1}^{\prime}}, \ldots, t_{r}^{\alpha_{r}^{\prime}}\right)},
$$

where $\beta=\alpha^{\prime}-\alpha$. This makes $\left\{N_{\alpha}\right\}$ into an inductive system. We have a commutative diagram as follows.

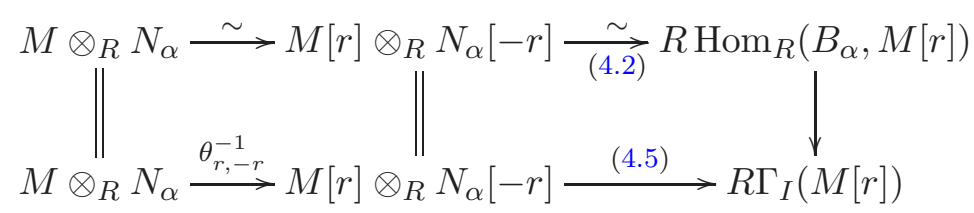

Consider the bottom row. Taking the 0th cohomology, applying $\lim _{\alpha}$ and using formulae (C.2) and (C.3) we get an isomorphism

$$
\underset{\alpha}{\lim }\left(M \otimes_{R} N_{\alpha}\right) \stackrel{\sim}{\longrightarrow} H_{I}^{r}(M)=H_{\widehat{I}}^{r}(\widehat{M}) .
$$

Setting $M=R$ we get

$$
\underset{\alpha}{\lim } N_{\alpha} \stackrel{\sim}{\longrightarrow} H_{I}^{r}(R)=H_{\widehat{I}}^{r}(\widehat{R})
$$




\section{P. SASTRY}

Note that in view of Remark C.1.1 and the sign implicit in $\theta_{r,-r}$, the composite map $M \otimes_{R} N_{\alpha} \rightarrow$ $\lim _{\longrightarrow}\left(M \otimes_{R} N_{\alpha}\right) \rightarrow H_{I}^{r}(M)$ is (using the notation of (4.1))

$$
m \otimes \frac{1}{\left(t_{1}^{\alpha_{1}}, \ldots, t_{r}^{\alpha_{r}}\right)} \mapsto\left[\begin{array}{c}
m \\
t_{1}^{\alpha_{1}}, \ldots, t_{r}^{\alpha_{r}}
\end{array}\right] .
$$

Finally, note that we have

$$
\left(M \otimes_{R} N_{\alpha}\right) \otimes_{R} R^{\prime}=M^{\prime} \otimes_{R^{\prime}} N_{\alpha}^{\prime},
$$

where, as before, $M^{\prime}=M \otimes_{R} R^{\prime}$.

Proposition C.4.1. The following diagrams commute.

a)

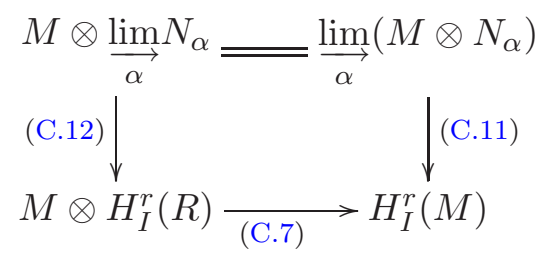

b)

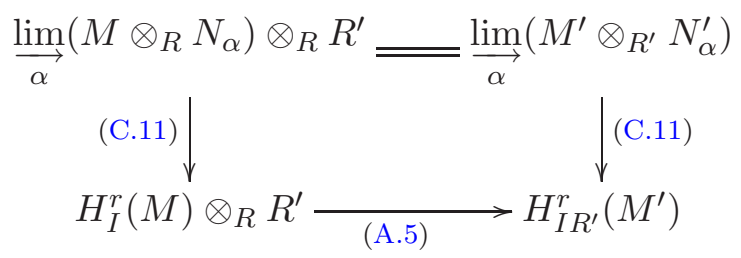

Proof. Both parts follow from the explicit descriptions of the maps involved using generalized fractions (Proposition C.3.1, Remark C.2.1, (C.13)).

Remark C.4.2. Suppose $M$ is a finitely generated $R$-module, and $\widehat{(\cdot)}$ denotes completion with respect to the $I$-adic topology. We have $R / \boldsymbol{t}^{\alpha} R=: B_{\alpha}=\widehat{R} / \boldsymbol{t}^{\alpha} \widehat{R}, I_{\alpha} / I_{\alpha}^{2}=\widehat{I}_{\alpha} / \widehat{I}_{\alpha}^{2}$, and $M \otimes_{R} N_{\alpha}=\widehat{M} \otimes_{\widehat{R}} N_{\alpha}$, and these identifications are compatible with the underlying inductive systems indexed by the $\alpha$. On taking direct limits, one checks that the resulting identification

$$
H_{I}^{r}(M)=H_{\widehat{I}}^{r}(\widehat{M})
$$

agrees with the one in Remark C.2.2 (use generalized fractions!).

\section{REFERENCES}

AJL99 L. Alonso Tarrío, A. Jeremías Lopez and J. Lipman, Duality and flat base change on formal schemes, in Studies in duality on Noetherian formal schemes and non-Noetherian ordinary schemes, Contemporary Mathematics, vol. 244 (American Mathematical Society, Providence, RI, 1999), 1-87.

Con B. Conrad, Deligne's notes on Nagata's compactification, Unpublished.

Con00 B. Conrad, Grothendieck duality and base change, Lecture Notes in Mathematics, vol. 1750 (SpringerVerlag, Berlin, 2000).

Del66 P. Deligne, Cohomologie à support propre et construction du foncteur $f^{!}$, Appendix to R. Hartshorne, Residues and duality, Lecture Notes in Mathematics, vol. 20 (Springer-Verlag, Berlin, 1966), 404-421.

GD61a A. Grothendieck and J. Dieudonné, Éléments de Géométrie Algébrique, vol. II, Publ. Math. Inst. Hautes Études Sci., no. 8 (1961).

GD61b A. Grothendieck and J. Dieudonné, Éléments de Géométrie Algébrique, vol. III, Publ. Math. Inst. Hautes Études Sci., no. 11 (1961), no. 17 (1963). 


\section{Base CHANGE FOR COHEN-MACAUlay MAPS}

GD64 A. Grothendieck and J. Dieudonné, Éléments de Géométrie Algébrique, vol. IV, Publ. Math. Inst. Hautes Études Sci., no. 20 (1964), no. 24 (1965), no. 28 (1966), no. 32 (1967).

Gro68 A. Grothendieck et al., Cohomologie locale des faisceuax cohérents et théorèmes de Lefschetz lacaux et globaux (North-Holland, Amsterdam, 1968).

Har66 R. Hartshorne, Residues and duality, Lecture Notes in Mathematics, vol. 20 (Springer-Verlag, Berlin, 1966).

Har77 R. Hartshorne, Algebraic geometry, Graduate Texts in Mathematics, vol. 52 (Springer-Verlag, Berlin, 1977).

HK90a R. Hübl and E. Kunz, Integration of differential forms on schemes, J. reine angew. Math. 410 (1990), 53-83.

HK90b R. Hübl and E. Kunz, Regular differential forms and relative duality for projective morphisms, J. reine angew. Math. 410 (1990), 84-108.

HS93 R. Hübl and P. Sastry, Regular differential forms and relative duality, Amer. J. Math. 115 (1993), 749-787.

Hub89 R. Hübl, Traces of differential forms and Hochschild homology, Lecture Notes in Mathematics, vol. 1368 (Springer-Verlag, New York, 1989).

Kle80 S. Kleiman, Relative duality for quasi-coherent sheaves, Compositio Math. 41 (1980), 39-60.

Lip79 J. Lipman, Double point resolutions of deformations of rational singularities, Compositio Math. 38 (1979), 37-43.

Lip84 J. Lipman, Dualizing sheaves, differentials and residues on algebraic varieties, Asterisque 117 (1984).

Lip87 J. Lipman, Residues and traces of differential forms via Hochschild homology, Contemp. Math. 61 (1987).

Lip99 J. Lipman, Non-Noetherian Grothendieck duality, in Studies in duality on Noetherian formal schemes and non-Noetherian ordinary schemes, Contemporary Mathematics, vol. 244 (American Mathematical Society, Providence, RI, 1999), 115-123.

Lip03 J. Lipman, Notes on derived categories and derived functors, Preprint at $<$ http://www.math.purdue.edu/ lipman/>.

LS92 J. Lipman and P. Sastry, Regular differentials and equidimensional scheme-maps, J. Algebraic Geom. 1 (1992), 101-130.

Lut93 W. Lütkebohmert, On compactification of schemes, Manuscr. Math. 80 (1993), 95-111.

Mat86 H. Matsumura, Commutative ring theory (Cambridge University Press, Cambridge, 1986).

Nag62 M. Nagata, Imbedding an abstract variety in a complete variety, J. Math. Kyoto Univ. 2 (1962), $1-10$.

Ne96 A. Neeman, The Grothendieck duality theorem via Bousfield's techniques and Brown representability, J. Amer. Math. Soc. 9 (1996), 205-236.

Ver69 J-L. Verdier, Base change for twisted inverse image of coherent sheaves, in Algebraic geometry (Oxford University Press, London, 1969), 393-408.

Pramathanath Sastry pramath@math.toronto.edu

Department of Mathematics, University of Toronto at Mississauga, 3359 Mississauga Road North, Mississauga, ON, L5L 1C6, Canada 